\title{
Study of an error in engine ECU data collected for in-use emissions testing and development and evaluation of a corrective procedure
}

\author{
Petr Sindler \\ West Virginia University
}

Follow this and additional works at: https://researchrepository.wvu.edu/etd

\footnotetext{
Recommended Citation

Sindler, Petr, "Study of an error in engine ECU data collected for in-use emissions testing and development and evaluation of a corrective procedure" (2007). Graduate Theses, Dissertations, and Problem Reports. 1840.

https://researchrepository.wvu.edu/etd/1840

This Thesis is protected by copyright and/or related rights. It has been brought to you by the The Research Repository @ WVU with permission from the rights-holder(s). You are free to use this Thesis in any way that is permitted by the copyright and related rights legislation that applies to your use. For other uses you must obtain permission from the rights-holder(s) directly, unless additional rights are indicated by a Creative Commons license in the record and/ or on the work itself. This Thesis has been accepted for inclusion in WVU Graduate Theses, Dissertations, and Problem Reports collection by an authorized administrator of The Research Repository @ WVU. For more information, please contact researchrepository@mail.wvu.edu.
} 
Study of an Error in Engine ECU Data Collected for In-Use Emissions Testing and Development and Evaluation of a Corrective Procedure

\title{
Petr Sindler
}

\author{
Thesis submitted to the \\ College of Engineering and Mineral Resources \\ at West Virginia University \\ in partial fulfillment of the requirements \\ for the degree of
}

\author{
Master of Science \\ in \\ Mechanical Engineering \\ Mridul Gautam, Ph.D., Chair \\ Gregory J. Thompson, Ph.D. \\ Benjamin C. Shade, Ph.D
}

Department of Mechanical and Aerospace Engineering

Morgantown, West Virginia

2007

Keywords: In-Use Emissions, ECU Communication, Communication Protocol, Time Stamp Error 


\begin{abstract}
Study of an Error in Engine ECU Data Collected for In-Use Emissions Testing and Development and Evaluation of a Corrective Procedure
\end{abstract}

\title{
Petr Sindler
}

A study was conducted to evaluate and correct possible inaccuracies in in-use emissions measurements, which were made with Portable Emissions Measurement Systems (PEMS). West Virginia University developed a PEMS termed the Mobile Emissions Measurement System (MEMS) for measurement of heavy duty on road diesel emissions and reporting them in brake specific units (g/bhp-hr). In particular, the study focused on inaccuracies in the engine Electronic Control Unit (ECU) broadcast data logged by the Dearborn Protocol Adapter (DPA) and the Data Link Monitor (DLM) software. Data for this study were collected both in the West Virginia University's Engine Emission Research Laboratory with engines running FTP cycles on a dynamometer, and during actual on-road tests conducted with the MEMS. The dynamometer test data show substantial and consistent inaccuracies of time stamps associated with the messages acquired with the USB DPA adapters while the DPA adapters with serial communication proved to be accurate. These time stamp inaccuracies associated with the USB DPA adapters result in erroneous evaluation of brake specific on-road emissions due to misalignment between the emission data and the power values acquired from the ECU. This time stamp error results in the USB DPA/DLM collected data file to be shorter, in terms of time, than the actual test. However, this time "shrinkage" can be readily corrected by linearly stretching the time array associated with the DLM collected data. The factor for the correcting stretch can be determined by aligning and matching the erroneous data with some independent but relevant measurement, such as $\mathrm{CO}_{2}$ mass emissions rate. This method for correcting ECU data reduced the average error in brake specific emissions from 6.24 percent to 0.13 percent, when compared to brake specific emissions reduced with serial DPA/DLM data. 


\section{Acknowledgements}

I would like express my appreciation to all who helped me to move along my educational journey. First of all, I would like to thank Dr. Mridul Gautam who convinced me to pursue Masters Degree, funded my studies during that time and was my advisor and the chair of my committee. I would like to thank also my other committee members, Dr. Gregory Thompson for suggesting this study and assisting with it, and Dr. Benjamin Shade, who answered for me numerous questions and helped me with assembly of this work.

There are countless people that helped me during my graduate work who I want to thank for doing that. I would like to thank to all the faculty, staff members and fellow students working at EERC and Mobile Lab as you all helped me with something at one time or another.

I would like to particularly thank Dan Carder who helped me more times than he would even want to remember. Also, thanks to Ron Jarrett, Tom McConnell, Josh Flaugher, Robin Ames and Michelangelo Ardanese, who helped me with acquiring data for my work. At last, not least, I would like to thank Raffaello Ardanese for writing the program that was used for this study. 
Table of Contents

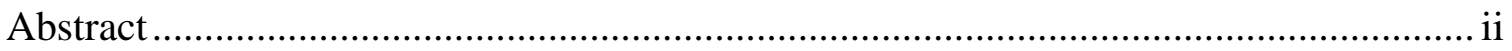

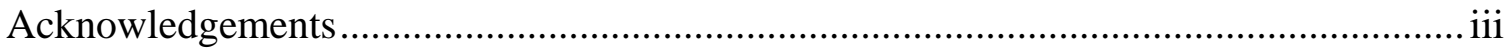

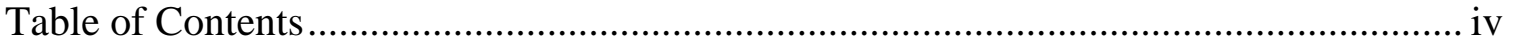

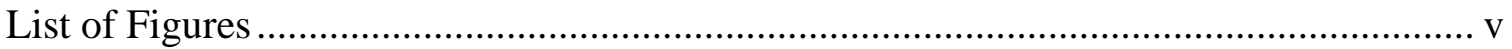

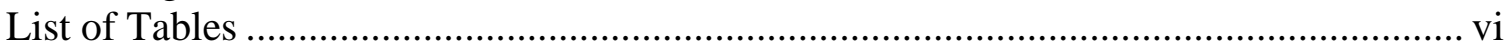

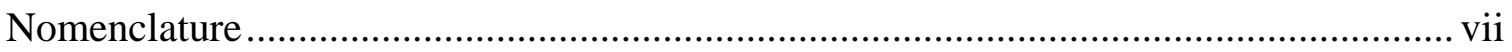

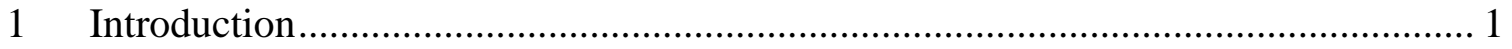

$1.1 \quad$ Methods of Engine Emissions Measurement.............................................. 2

1.2 Overview of Methods of Power Evaluation for On-Road Testing ..................... 3

1.3 Brake Specific Emission Data Compilation ...................................................... 5

1.4 Alignment of Necessary Information............................................................. 5

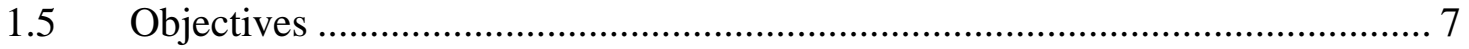

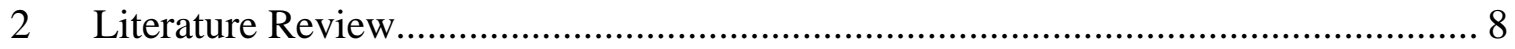

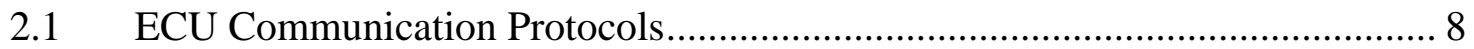

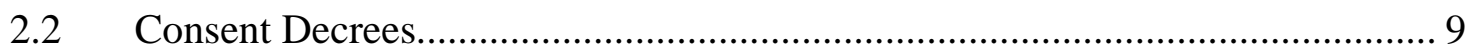

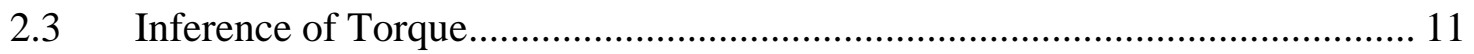

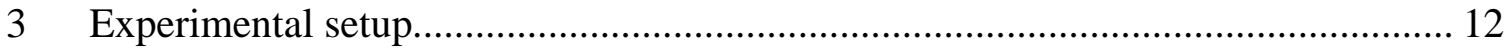

3.1 Laboratory Setup.................................................................................. 12

On Road Test Setup ........................................................................... 21

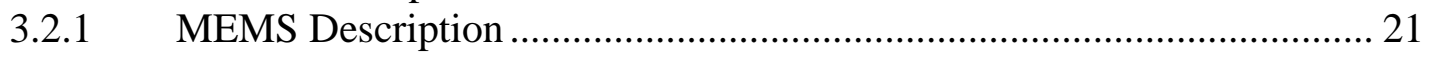

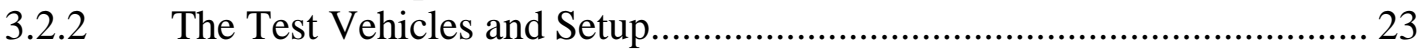

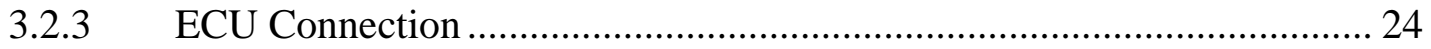

3.2.4 Details on Connecting to J1939 CAN Link ............................................. 26

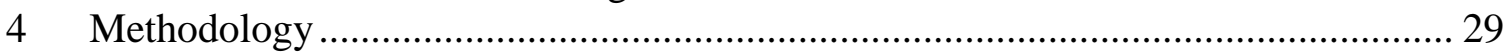

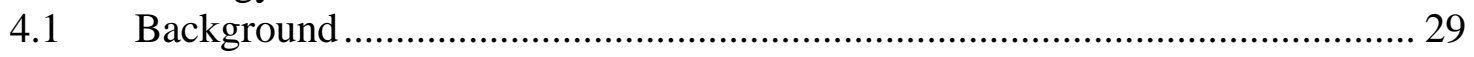

4.1.1 Difficulties With On-Road Data Acquisition ....................................... 29

4.1.2 Description of Error Encountered in MEMS Data .................................. 29

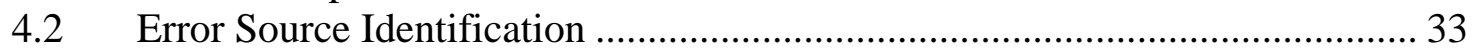

E.3 Error Correction ............................................................................................ 34

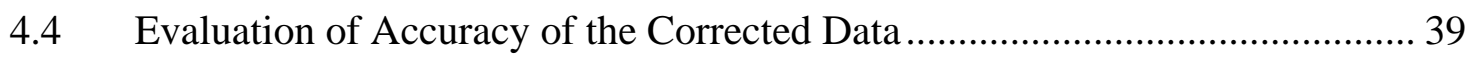

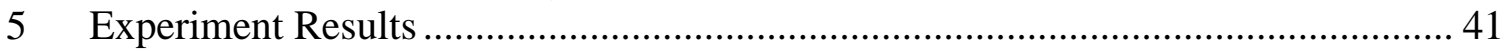

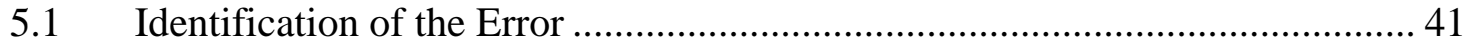

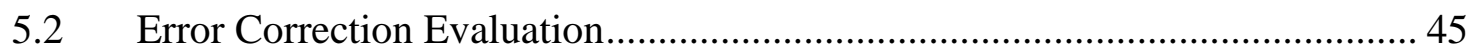

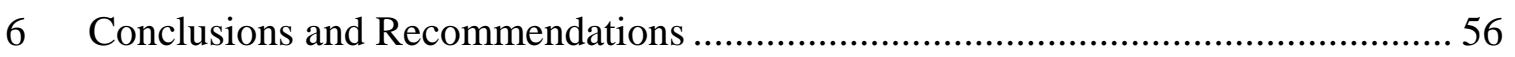

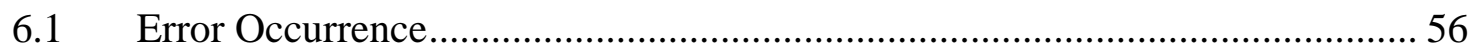

6.2 Error Correction for MEMS Data Reduction Application................................ 57

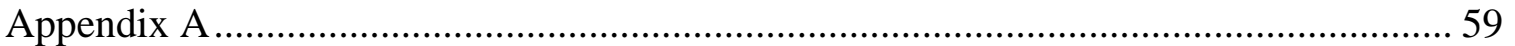

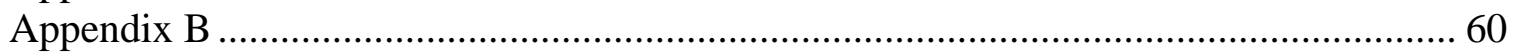

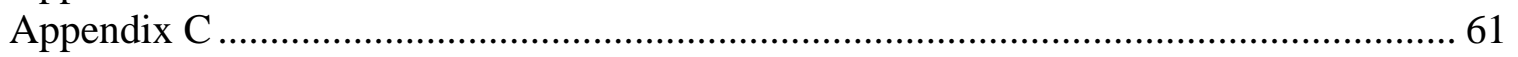

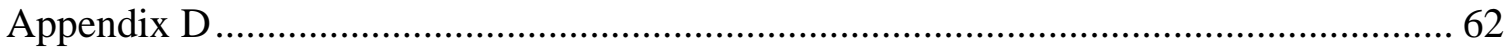

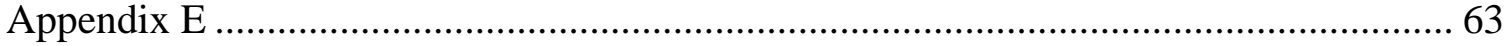




\section{List of Figures}

Figure 1 Aligned Data Sets and Their Product ............................................................. 6

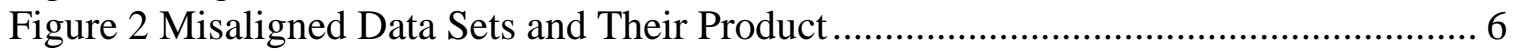

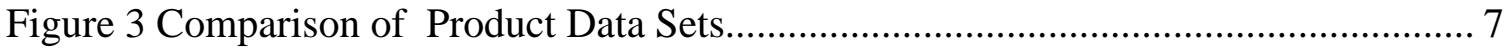

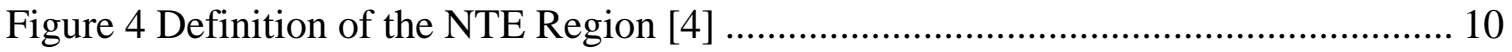

Figure 51992 DDC Series 60 Engine Mounted on a Dynamometer .............................. 13

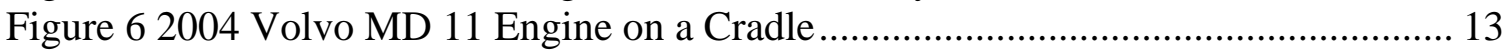

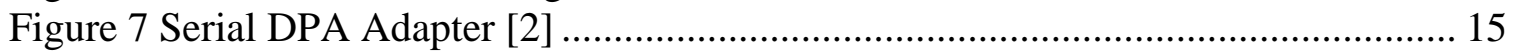

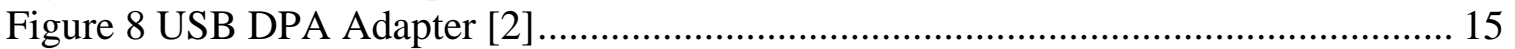

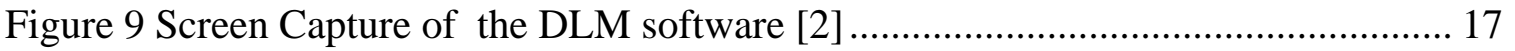

Figure 10 DB15 Connector with Indicated Numbering Order [5].................................. 18

Figure 11 Pin-out of the ECM connector on the 1992 DDC 60 Engine [13 ] .................. 19

Figure 12 Connectors B (Left) and A (Right) On the Volvo MD 11 Engine ECU .......... 20

Figure 13 Pin Numbering Pattern of Volvo MD 11 Engine ECU Connectors................. 20

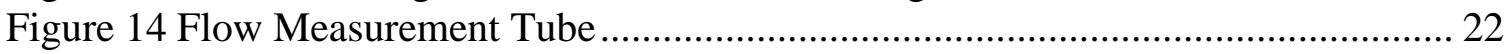

Figure 15 MEMS Data Acquisition (left) and Emission (right) Boxes .......................... 23

Figure 16 Deutsch 9-Pin Connector - Pin Letter Marking Assignment [6]..................... 24

Figure 17 Deutsch 6-Pin Connector - Pin Letter Marking Assignment [5] ..................... 25

Figure 18 Deutsch 3-Pin Connector - Pin Letter Marking Assignment [7] .................... 25

Figure 19 Controller Area Network J1939 Architecture [11] ......................................... 27

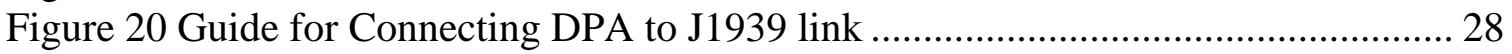

Figure 21 Misaligned Engine Power and Emission Data ........................................... 31

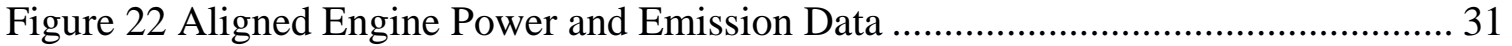

Figure 23 MEMS Test Data Aligned at the Beginning of the Test ................................ 32

Figure 24 MEMS Test Data Showing Discrepancy at the End of the Test ..................... 32

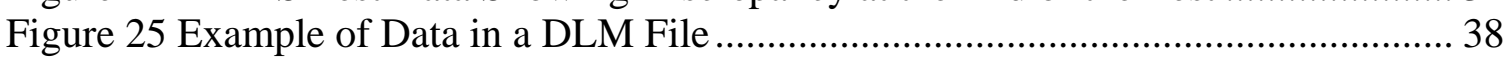

Figure 26 Engine speed Data Collected by the Lab, Serial DPA and USB DPA on 1708

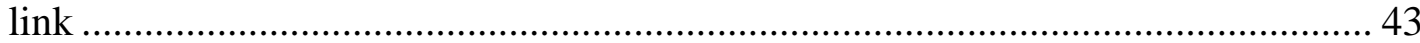

Figure 27 Engine speed Data Collected by the Lab, Serial DPA and USB DPA on 1939

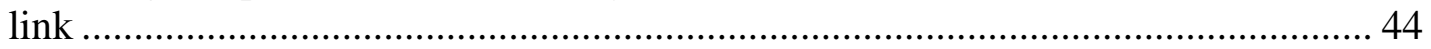

Figure 28 Regression Analysis of Engine Power with Erroneous USB Data and the Serial

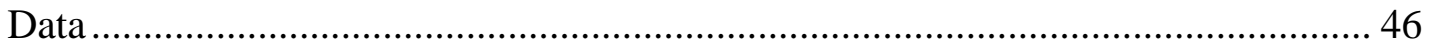

Figure 29 Regression Analysis of Corrected USB and Serial Engine Power Data ......... 46

Figure 30 Regression Analysis of Filtered Corrected Engine Power Data...................... 48

Figure $3130 \mathrm{sec}$ NTE Maximum MEXA NOx Error - Dependence on Test Length and

Amount of "Shrinkage" in USB DLM Data ........................................................... 50

Figure 32 Continuous NTE Maximum MEXA NOx Error - Dependence on Test Length and Amount of "Shrinkage” in USB DLM Data ................................................. 51

Figure 33 Error in Maximum MEXA NOx Brake Specific NTE Emissions ................... 52

Figure 34 Detail of Error in Maximum MEXA NOx Brake Specific NTE Emissions .... 52 


\section{List of Tables}

Table 1 Heavy-Duty Diesel Emission Standards for Years 1988-2003, g/bhp-hr [1] ........ 1 Table 2 Heavy-Duty Diesel Emission Standards for Years 2004 and up, g/bhp-hr [1]...... 2

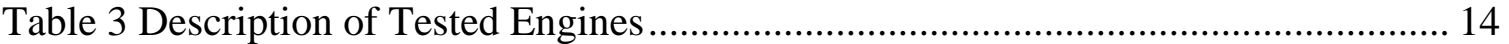

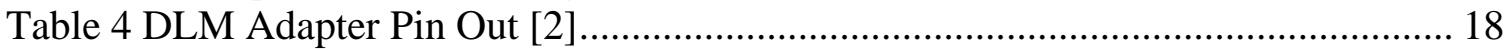

Table 5 Volvo MD 11 ECU Communication Link Pin-Out ........................................... 21

Table 6 Pin-out of the Various Deutsch Connectors Used for ECU Communication...... 25

Table 7 Summary of Regression Analyses Trend Line Equations of Serial and Corrected

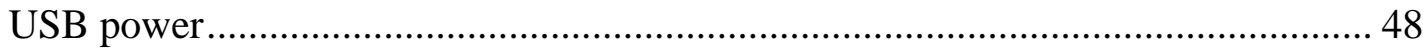

Table 8 Vehicles and Engines with Corresponding Test Numbers ................................ 53

Table 9 Summary of NOx Max Test Results for Vehicles with 2001 Manufacturer A

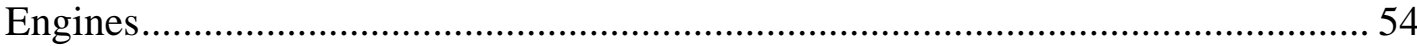

Table 10 Summary of NOx Max Test Results for a Vehicle with the 2002 Manufacturer

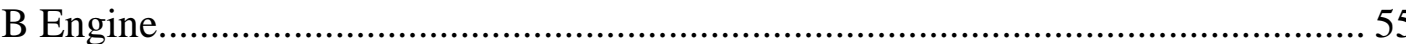




\author{
Nomenclature \\ CAN \\ CFR \\ $\mathrm{CO}$ \\ $\mathrm{CO}_{2}$ \\ DLM \\ DPA \\ ECM \\ ECU \\ EERL \\ EPA \\ FTP \\ HDDE \\ HC \\ MEMS \\ MID \\ NDIR \\ $\mathrm{N}_{15 \% \text { ESC }}$ \\ $\mathrm{N}_{\text {hi }}$ \\ $\mathrm{N}_{\mathrm{lo}}$ \\ NMHC \\ NOx \\ NTE \\ PEMS \\ PID \\ PM \\ SHDDE \\ WVU \\ Controller Area Network \\ Code of Federal Regulations \\ Carbon Monoxide \\ Carbon Dioxide \\ Data Link Monitor \\ Dearborn Protocol Adapter \\ Electronic Control Module \\ Electronic Control Unit (Same as ECM- Matter of Preference) \\ Engine Emission Research Laboratory at WVU \\ Environmental Protection Agency \\ Federal Transient Procedure \\ Heavy Duty Diesel Engine \\ Hydrocarbons \\ Mobile Emissions Measurement System \\ Message Identifier \\ Non Dispersive Infra Red (analyzer) \\ 15\% European Stationary Cycle Speed \\ Highest Engine Speed at which 70\% of Maximum Power Occurs \\ Lowest Engine Speed at which 50\% of Maximum Power Occurs \\ Non Methane Hydrocarbons \\ Oxides of Nitrogen \\ Not To Exceed (Zone of engine operation) \\ Portable Emissions Measurement System \\ Parameter Identifier \\ Particulate Matter \\ Settling HDDE (manufacturers) \\ West Virginia University
}




\section{Introduction}

Environmental concerns in the past few decades resulted in increasing efforts by the United States (US) Environmental Protection Agency (EPA) to reduce harmful emissions from all sorts of human activities. A substantial portion of airborne emissions in the United States are emitted by diesel powered heavy duty vehicles operating on road [15]. US EPA has required manufacturers of heavy duty diesel engines (HDDE) to reduce the exhaust emissions by means of work specific emission limits. Currently, the emissions of carbon dioxide $\left(\mathrm{CO}_{2}\right)$ are not regulated. Carbon dioxide is an unavoidable product from combustion of carbon-based fuel and the rate of its emission is directly proportional to fuel consumption. Therefore, the manufacturers are self motivated to keep the $\mathrm{CO}_{2}$ emissions low for the sake of good fuel economy and consequent consumer satisfaction. Regulated exhaust emissions from heavy duty diesel engines are carbon monoxide (CO), hydrocarbons (HC), oxides of nitrogen (NOx) and particulate matter (PM). A summary of the heavy duty diesel engine emission standards can be found in Table 1 and Table 2.

Table 1 Heavy-Duty Diesel Emission Standards for Years 1988-2003, g/bhp-hr [1]

\begin{tabular}{|c|c|c|c|c|}
\hline Year & HC & CO & NOx & PM \\
\hline 1988 & 1.3 & 15.5 & 10.7 & 0.60 \\
\hline 1990 & 1.3 & 15.5 & 6.0 & 0.60 \\
\hline 1991 & 1.3 & 15.5 & 5.0 & 0.25 \\
\hline 1994 & 1.3 & 15.5 & 5.0 & 0.10 \\
\hline 1998 & 1.3 & 15.5 & 4.0 & 0.10 \\
\hline
\end{tabular}

For 2004 model year, the engine manufacturers were allowed to certify their engines to either of the two options listed in Table 2. In 1998 the EPA and six settling heavy duty diesel engine (SHDDE) manufacturers entered Consent Decrees which required even stricter emission standards. Most of the (SHDDE) manufacturers were forced to comply with 2004 emissions by October 2002. Additional details regarding 
Consent Decrees can are discussed in Section 2.2. The 2007 emission limit of

$\mathrm{NMHC}+\mathrm{NOx}$ is not rigid for all engines as there is a phase-in period up to year 2010 at which point every manufactured engine will have to comply [16].

Table 2 Heavy-Duty Diesel Emission Standards for Years 2004 and up, g/bhp-hr [1]

\begin{tabular}{|c|c|c|c|c|c|}
\hline Year & HC & CO & NMHC+NOx & NMHC & PM \\
\hline 2004 opt.1 & 1.3 & 15.5 & 2.4 & n/a & 0.1 \\
\hline 2004 opt.2 & 1.3 & 15.5 & 2.5 & 0.5 & 0.1 \\
\hline 2007 & 1.3 & 15.5 & 0.2 & 0.14 & 0.01 \\
\hline
\end{tabular}

\subsection{Methods of Engine Emissions Measurement}

In the past, the emissions produced by a heavy duty diesel engine were primarily evaluated in a testing laboratory where it would be loaded by a dynamometer and the exhaust emissions would be measured using a total-exhaust dilution tunnel under conditions specified by the EPA. The specifics of the laboratory design and testing procedures are outlined in the Code of Federal Regulations (CFR), Title 40, Part 86, Subpart N. This method, although producing very accurate and repeatable data, did not faithfully represent real world emissions, nor was it applicable to ensure compliance of emissions of engines mounted in vehicles in service. An approximation of on-road testing of in-use engines is the use of a chassis dynamometer that allows the whole vehicle to be placed on the test bed, without the engine removal, and tested in a manner that would simulate the vehicle being driven along some prescribed route. However, this testing procedure still does not produce real- world emission data. A vehicle tested on a chassis dynamometer still operates in somewhat controlled environment and conditions and follows a route, which is supposed to approximate certain traffic conditions that are thought to be present in a real-world road traffic. Typically, due to the constraints of chassis dynamometer design, the loads applied to the vehicle are low for simulation of 
rolling and aerodynamic resistance. Conditions of operation in hilly terrain, such as a continuous heavy load and motoring are rarely applied. To obtain an accurate picture of exhaust emissions produced by engines operating in the vehicles in use, the emissions measurement system needs to be placed on the vehicle and collect emissions data while the vehicle is driven over the road. One such system, called the Mobile Emission Measurement System (MEMS), was developed at WVU, and has been used to measure on road emissions of heavy duty diesel engines as per Consent Decrees between the EPA and the major U.S. heavy duty engine manufacturers [8]. MEMS is a portable system that can be installed on the vehicle being tested and is capable of quantifying brake specific mass rate of emissions of $\mathrm{NOx}$ and $\mathrm{CO}_{2}$.

One of the challenges of using a portable emission measurement system is to evaluate the amount of work done by the engine, while the vehicle is driven on the road. Evaluation of work is necessary in order for the emission values to be reported on a brake specific basis, which actually means in mass of emission constituent per unit work and is reported in units of g/bhp-hr or g/kW-hr. [4]. Work is usually determined by measuring of engine torque and engine speed.

Measurement of engine torque and speed is also important for determining the Not to Exceed (NTE) engine operation. The NTE operation region is defined in the Consent Decrees as an engine operation region in which in-use emissions must be evaluated. The significance and definition of NTE engine operation is described in Section 2.2.

\subsection{Overview of Methods of Power Evaluation for On-Road Testing}

During the development of MEMS, several methods were considered for evaluation of engine-generated power. One of the considered methods was measuring a load induced strain somewhere in the driveline of the vehicle, and correlating it to the engine torque. The most practical realization of this method is to place a strain gage on 
the drive shaft to measure the twist of the shaft under a load, which can be through calibration correlated to the torque transmitted through the shaft. This information, along with the information of the rotational speed of the shaft, can be used to evaluate the power transmitted through the shaft. The shaft speed can be either measured directly with some optical or magnetic transducer, or calculated from engine speed and transmission ratio. This method of engine power evaluation however, is not very appealing as it requires additional equipment to be mounted to the vehicle that is not only fairly expensive, but also time consuming to install and calibrate. Another undesirable issue with this method is the unaccounted power loss due to transmission inefficiencies between the flywheel of the engine and the point of torque measurement.

A second possible method to evaluate the engine power is to correlate the power to the road load imposed on the vehicle [3]. Road load is the power requirement of the vehicle in motion during hill climbing and acceleration, and losses related to aerodynamic drag, rolling resistance and driveline inefficiencies. This method either requires obtaining large amounts of vehicle specific component information such as coefficients of drag and tire resistance or conducting additional tests in order to determine the road load imposed on the vehicle.

The power evaluation method that was chosen for the WVU built MEMS was to log the information broadcasted by the engine Electronic Control Unit (ECU). The ECU continuously broadcasts numerous parameters intimately related to engine’s operation, some of which can be used to evaluate the engine power. The parameters useful for calculating power are engine speed and torque. The newer, post-Consent Decree engine ECUs broadcast engine torque, whereas the older engine ECUs broadcasted percent load which was correlated to engine torque [4]. The ECU data broadcast conforms to standardized industry formats, the SAE J1708/J1587 and J1939 protocols. Specifications 
and details of these protocols are discussed in Section 2.1. In order to log the engine ECU broadcast, a hardware interface must be placed between the data acquisition computer and the engine ECU to facilitate the communication between the two incompatible systems. The hardware interface selected for MEMS application was a Dearborn Protocol Adapter (DPA) manufactured by Dearborn Group Inc. along with the Data Link Monitor (DLM) software provided for logging the broadcast data.

\subsection{Brake Specific Emission Data Compilation}

Calculating brake specific engine emission values requires combining several bits of information. The first part of information needed is the concentrations of emission constituents. This information is obtained by emission gas analyzers. The second piece of information is the total engine exhaust mass-rate, which is measured by an averaging differential pressure device in the MEMS. Using these data, a mass rate of emission constituent is calculated. The emissions mass rate is then divided by the work output of the engine to obtain the brake specific emission values.

\subsection{Alignment of Necessary Information}

During an engine emission test, all required parameters are measured and recorded simultaneously with the intent to calculate instantaneous brake specific emissions throughout the test. However, owing to the design of the measurement and data acquisition equipment, there may be a misalignment of recorded data on a time basis. Therefore, data from an engine emission test must be correctly aligned to assure that the emission values, calculated at any point in time of the test, correspond to the measured data, which uniquely belong to that engine event. The alignment is usually done by visual inspection of plotted data, where a rise in one parameter corresponds to a rise in another. When the data are aligned at the one point of the test, due to time continuity, they generally correspond to each other throughout the whole test. However, some of the in- 
use emissions tests have produced data series that could not be readily aligned due to an inconsistent time shift of collected information. As a result, brake specific emission data would be affected.

To illustrate this phenomenon, consider two generic data sets called Parameter 1 and Parameter 2. These data sets are used to generate, through a functional relationship, a product data set simply called the Product. Figure 1 shows a plot of a section of these data sets aligned with the resulting product. Figure 2 shows a plot of the same data sets when they are misaligned.

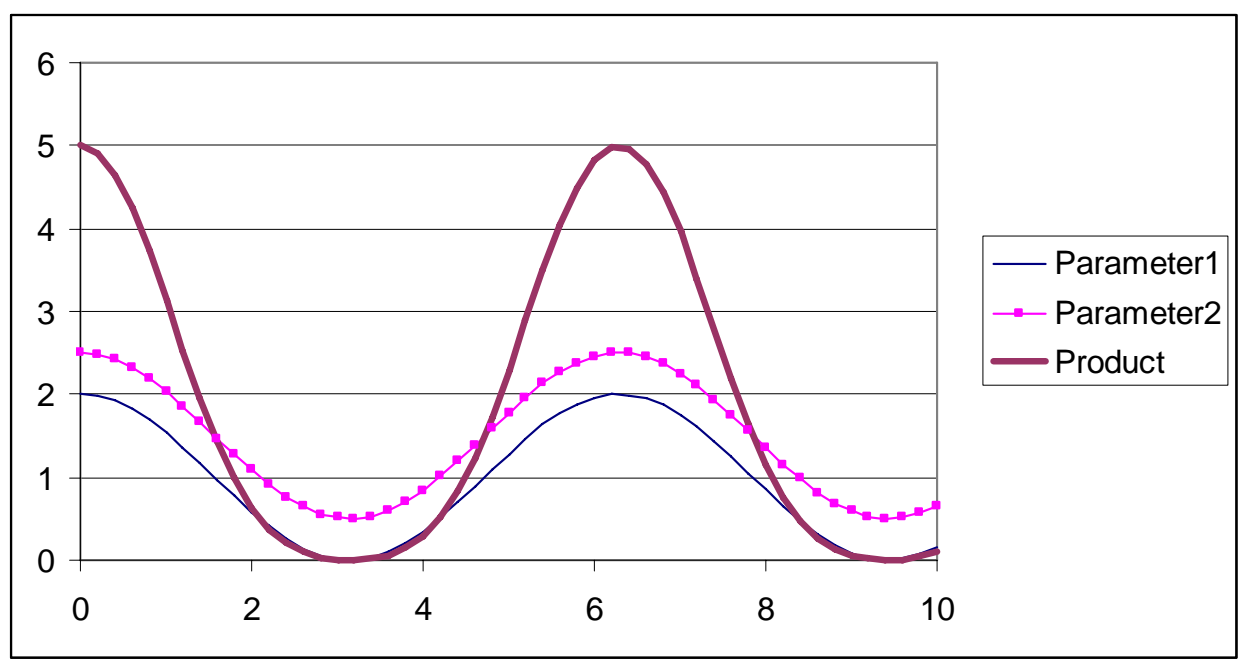

Figure 1 Aligned Data Sets and Their Product

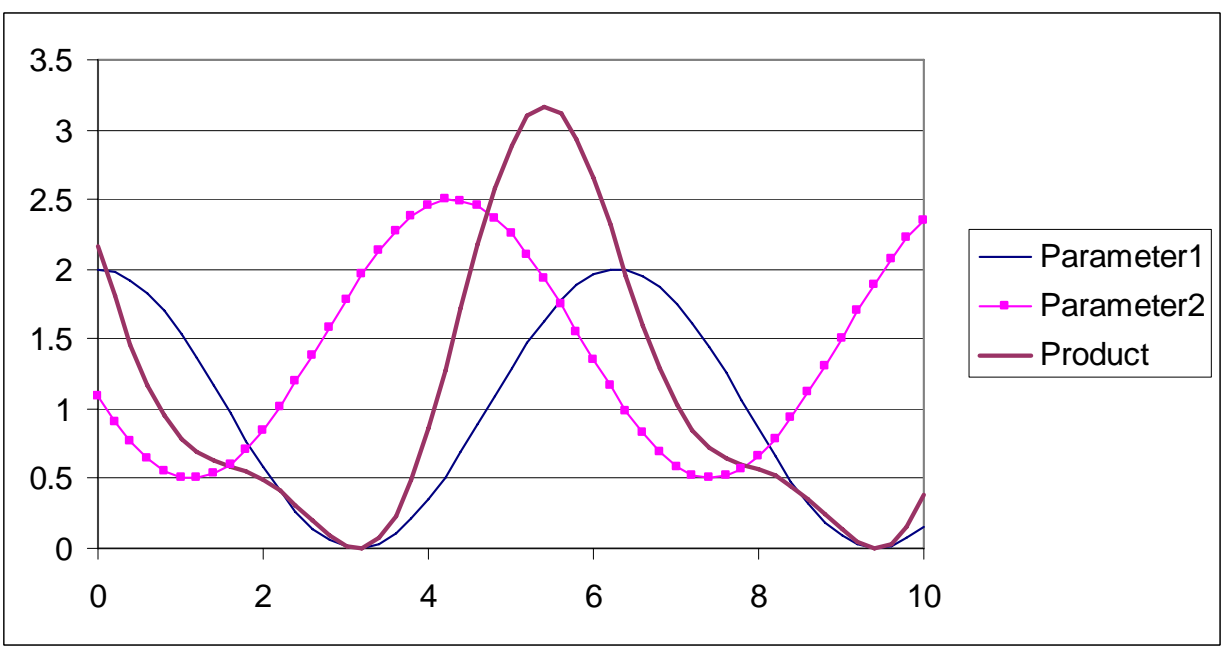

Figure 2 Misaligned Data Sets and Their Product 
These figures demonstrate how the two data sets can produce different products with different time alignment. The difference between the two resulting products is emphasized in Figure 3.

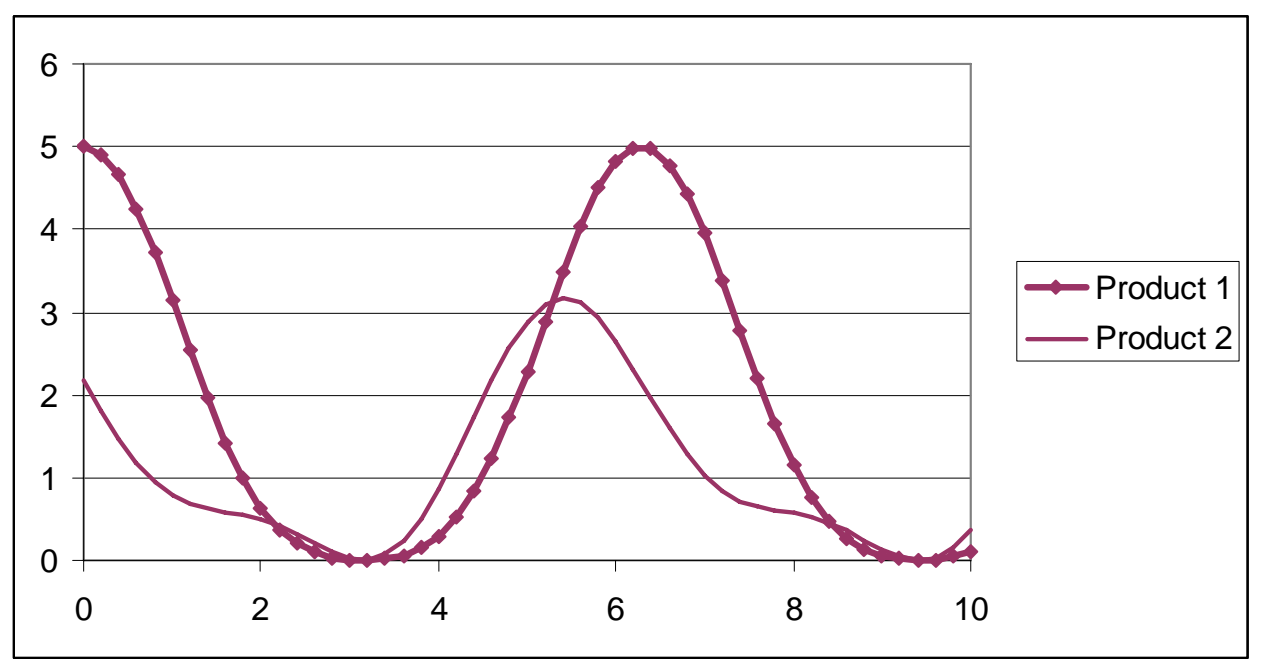

Figure 3 Comparison of Product Data Sets

The problem with time alignment in some of the above mentioned in-use emissions test data sets was identified as an error associated with the data acquisition of ECU data using the DPA adapters and DLM software.

\subsection{Objectives}

The objectives of this study were to find the source of error associated with timing of engine parameter data logged using the DPA adapters and DLM software, determine combinations of hardware, settings and conditions that cause and influence the error, and if possible, find solutions to this problem. These solutions could range from identifying a valid method of collecting ECU data to avoid misaligned data to creating a methodology for correcting data that was already collected. 


\section{Literature Review}

\subsection{ECU Communication Protocols}

Currently two protocols are used for ECU communication on heavy duty diesel engines used in United States. These protocols are SAE J1587 (operating on hardware conforming to J1708 specifications) and SAE J1939. SAE provides hardware and software specifications in its Surface Vehicle Standards publications [10-13]. There is a large amount of engine and vehicle information broadcast by the ECU, some of which is publicly available and some that is manufacturer proprietary. The ECU communication links can be accessed with interface hardware conforming to the J1708 or J1939 standards. Pieces of information, that are of prime interest for emissions testing are engine operation parameters, such as engine speed, engine torque, and if the engine torque is not available, such as in cases of older - pre Consent Decree - engines, the engine percent load. These parameters are useful for determining the engine work output for evaluating brake specific emissions for on-road in-use emission testing.

The J1708 data bus was issued in 1986 [10,11,15]. It operates on a pair of wires up to 40 meters long and will support a minimum of 20 nodes with ECUs. The data transfer rate is $9.6 \mathrm{kbits} / \mathrm{s}$. The communication protocol is specified as J1587. It is a serial data transfer with messages up to 21 characters long. Each message starts with Message Identifier (MID), followed by Parameter Identifiers(PID) and the actual parameter values.

The J1939 CAN data bus was designed to replace the J1708/J1587 bus/protocol[12,13,15]. The first J1939 standards were issued in 1994. It is a Controller Area Network (CAN) that operates on a backbone consisting of a shielded twisted pair of wires (denoted as $\mathrm{CAN}_{\mathrm{hi}}$ and $\mathrm{CAN}_{\mathrm{lo}}$ ) up to 40 meters long, terminated at each end with a 120 ohm resistor. The termination resistors provide the system with high signal to noise 
ratio. The data transfer rate is $250 \mathrm{kbits} / \mathrm{s}$, which is significantly higher than on the J1708 bus.

\subsection{Consent Decrees}

In 1998 the United States government, at the request of Environmental Protection Agency, filed complaints against six heavy duty diesel engine manufacturers for violating the Clean Air Act [4]. The manufacturers were allegedly equipping engines with emission "Defeat Devices" that allow the engines to pass the FTP tests but employ creative engine control strategies during certain modes of in-use operation. These strategies, although intended for improving fuel economy, may have adverse effect on NOx emissions. The manufacturers and the US government settled and entered the Consent Decrees without any trials. The Consent Decrees outline numerous details of post settlement engine specifications regarding emission control strategies, older engine remanufacturing and other orders that manufacturers must follow in their future conduct

of business. The manufacturers agreed to invest into a program of in use emission testing of their product and conduct on road testing. Instead of developing their own on-road testing equipment and procedures, the manufacturers contracted WVU to do so. WVU developed the Mobile Measurement Emission System that was approved by EPA for on road testing [8]. Part of the Consent Decrees was that manufacturers make provision for ECU broadcast engine torque, engine speed and injection timing updated at minimum of $5 \mathrm{~Hz}$. These engine torque and engine speed parameters are important for on-road emission testing. They are used for evaluating engine power, which is necessary for calculation brake specific emissions. Also, these parameters are needed for determining the Not To Exceed (NTE) engine operation, which is another specification outlined in the Consent Decrees. 
NTE is a region of engine operation, during which on-road emissions are measured. The engine has to operate in this region for at least 30 second continuously to calculate valid NTE emissions. Figure 4 shows the boundaries of the NTE operation region on an engine map. The low engine speed boundary is called 15\%ESC speed and is calculated by Equation 1, where $\mathrm{N}_{\mathrm{lo}}$ is defined as the lowest engine speed at which $50 \%$ of maximum power occurs. $\mathrm{N}_{\mathrm{hi}}$ is defined as the highest speed at which $70 \%$ of maximum power occurs.

$$
N_{15 \% E S C}=N_{l o}+0.15\left(N_{h i}-N_{l o}\right)
$$

Equation 1

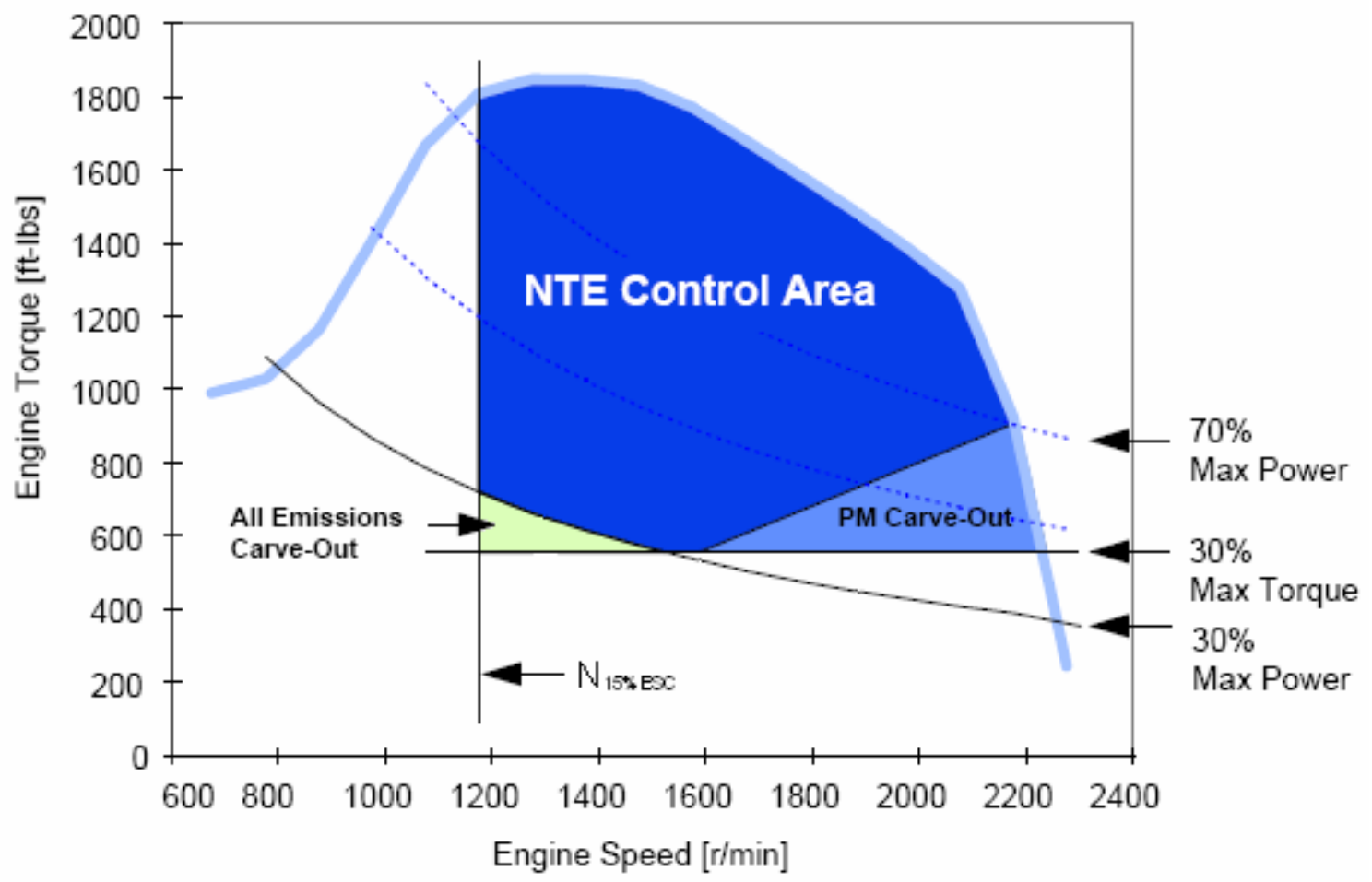

Figure 4 Definition of the NTE Region [4]

The in-use emission limit for brake specific 30 second NTE NOx emission was determined to be the 1.25 (or 1.75 - depending on the year of manufacture) times the FTP certification limit plus $0.5 \mathrm{~g} / \mathrm{bhp}-\mathrm{hr}$. The additional $0.5 \mathrm{~g} / \mathrm{bhp}-\mathrm{hr}$ is a measurement allowance to compensate for on-road measurement inaccuracies [17]. 


\subsection{Inference of Torque}

Although the MEMS was developed to satisfy the in-use emissions requirements as outlined in the Consent Decrees, some development work was done for testing preConsent Decree engines. A problem with the pre-Consent Decree engines was that the ECU broadcasted only J1587 protocol data that generally do not contain a readily useable torque value. The engine speed parameter is broadcasted at a frequency of $10 \mathrm{~Hz}$ with sufficient resolution. However, the engine torque is generally not publicly available on the pre-Consent Decree J1587 protocol. A study was conducted at WVU to evaluate the accuracy of engine torque inferred from engine percent load, a parameter that is included in engine ECU broadcast [4]. The study showed that the error in the power derived with this method was on the order of 5 to 10 percent over a 30 second NTE window with an engine exercised through the FTP cycle. However, the cumulative energy over the whole 20 minutes of the FTP cycle showed much better results; the error was less then 1 percent in comparison to the engine dynamometer measurement. However, this was with a lug curve generated on the engine dynamometer. This integrated error may be higher if a manufacturer-supplied engine lug curve was used for an in use test. 


\section{Experimental setup}

This experimental study was conducted both in the engine test laboratory and during on road testing of vehicles with the MEMS.

\subsection{Laboratory Setup}

The first part of the study focused on determining what conditions or hardware combinations cause the timing error observed in some of the in-use test as mentioned earlier. These experiments were conducted in the West Virginia University’s Engine Emission Research Laboratory (EERL). This laboratory is equipped with dynamometers and a full flow dilution tunnel sampling system as specified by CFR 40 Part 86 Subpart $\mathrm{N}$. The part of the engine laboratory that was important for this study was an engine dynamometer with its controller and a data acquisition system collecting engine operation information such as speed, torque, temperatures, etc.

Two different engines were used for this study. One engine was a 1992 Detroit Diesel Corporation (DDC) Series 60 and the other was a 2004 Volvo MD11, both heavy duty diesel engines. The ECU of the DDC 60 broadcast data using the J1587 protocol, and the MD 11 was a newer engine and its ECU broadcast both J1587 and J1939 protocol data. The detailed description and specifications of these engines can be found in Table 3. For these experiments, the type of engine used was not important as long as the engines were of a newer make; hence electronically controlled with an ECU broadcasting J1939 or/and J587 protocol data. Figure 5 shows the DDC 60 engine in the laboratory test cell attached to the dynamometer, while Figure 6 shows the Volvo MD 11 engine on an engine stand. 


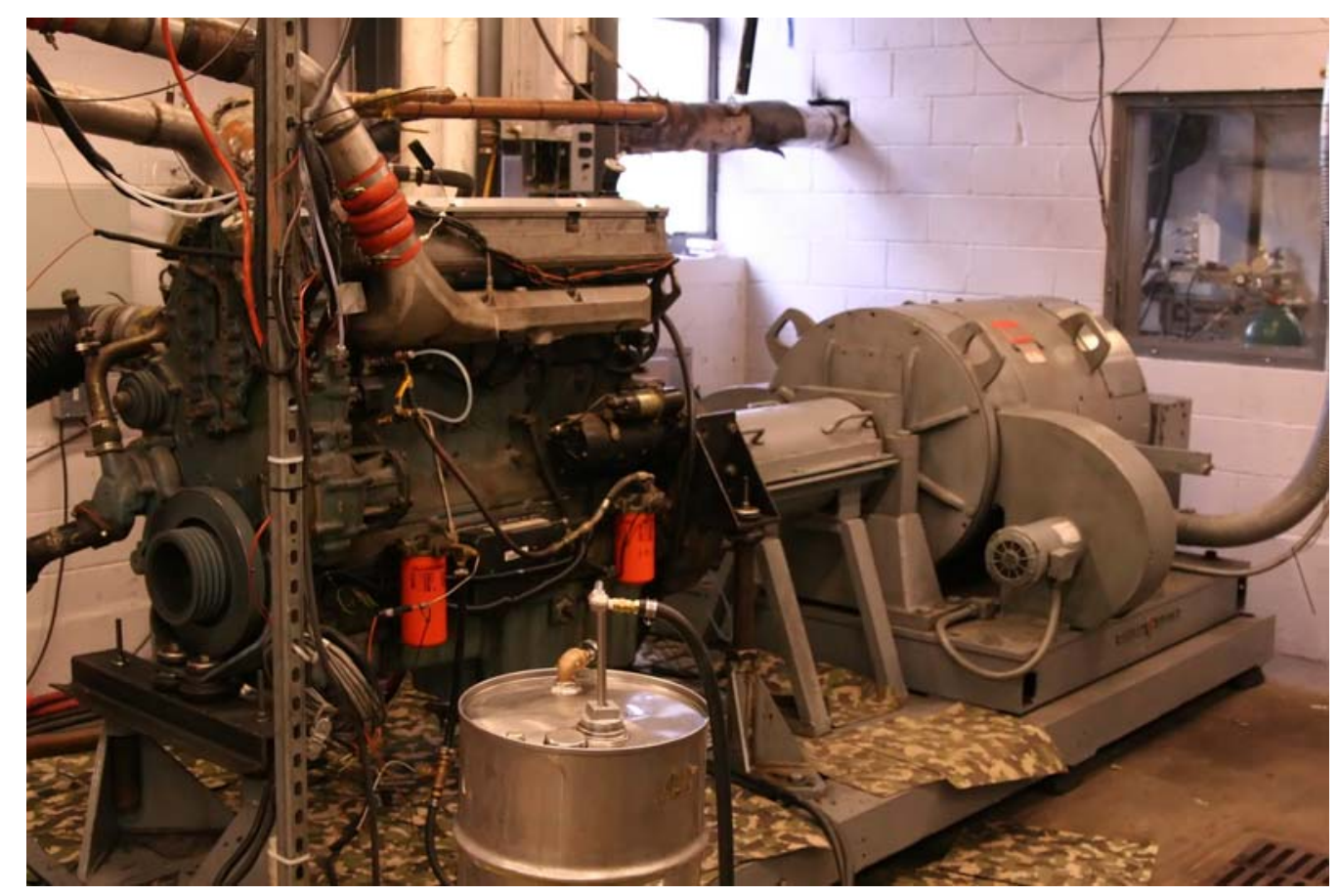

Figure 51992 DDC Series 60 Engine Mounted on a Dynamometer

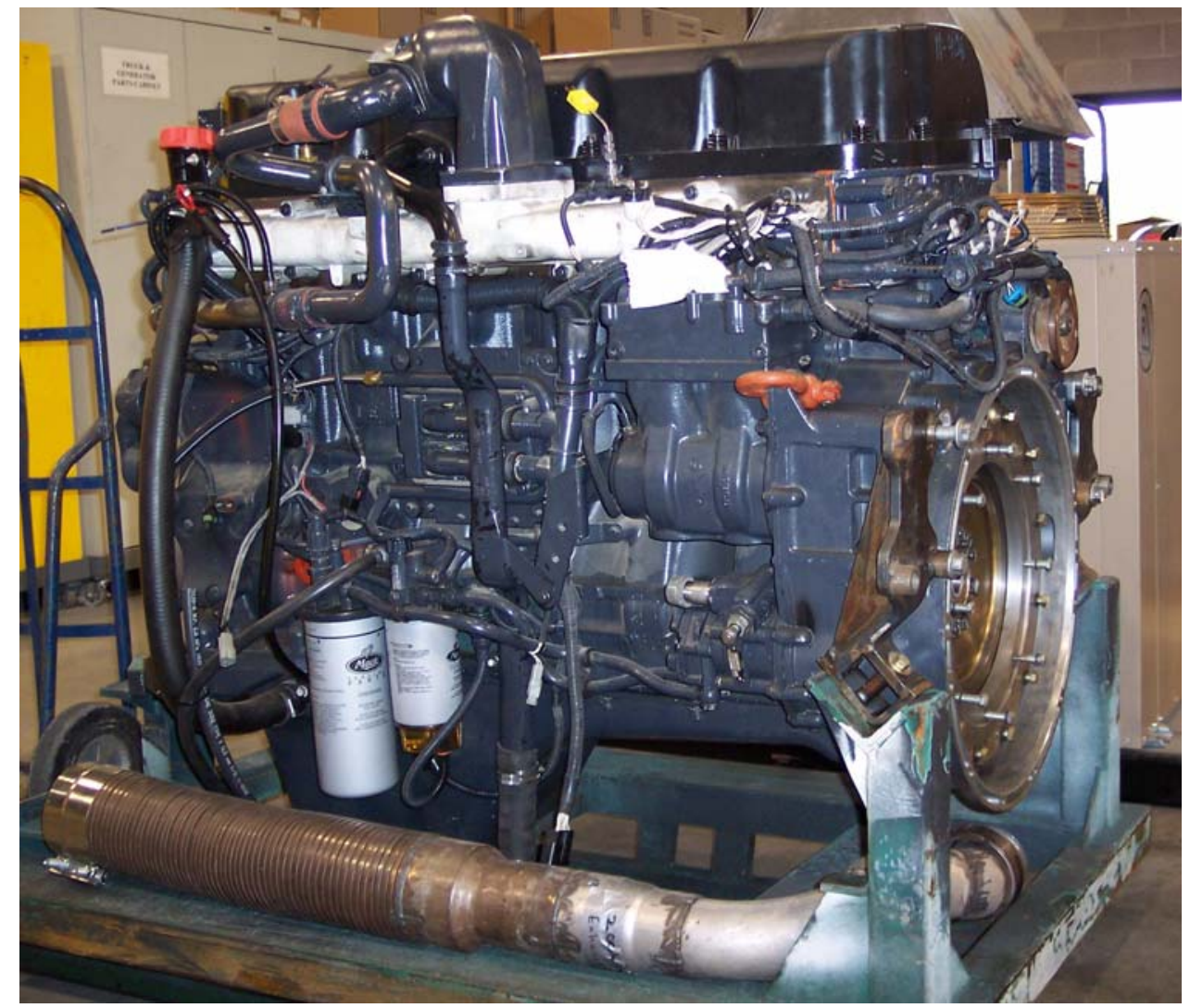

Figure 62004 Volvo MD 11 Engine on a Cradle 
Table 3 Description of Tested Engines

\begin{tabular}{|c|c|c|}
\hline Engine Manufacturer & Detroit Diesel Corp. & Volvo Truck Corp. \\
\hline Engine Model & Series 60 & MD 11 \\
\hline Model Year & 1992 & 2004 \\
\hline Displacement (liters) & 12.7 & 10.8 \\
\hline Power Rating (hp) & $360 @ 1820 \mathrm{rpm}$ & $370 @ 1800 \mathrm{rpm}$ \\
\hline Configuration & Inline 6 & Inline 6 \\
\hline Bore(in) X Stroke(in) & $5.12 \times 6.3$ & $4.84 \times 5.98$ \\
\hline Induction & Turbocharged, Intercooled & Turbocharged, Intercooled \\
\hline Cycle Description & 4 Stroke & 4 Stroke \\
\hline Injection & Direct, Electronic & Direct, Electronic \\
\hline ECU Communication Link & 1587 & 1587,1939 \\
\hline
\end{tabular}

The goals of this study were to collect engine parameter data broadcasted by the ECU using the DPA / DLM equipment and to compare these data to those collected by the laboratory. Multiple DPA adapters were obtained, some serial and some USB versions. In particular, models DG-DPA III Plus/T and DG-DPA 4 USB were used as these are the units used with the WVU MEMS for on-road emission testing. The DPAs are simple to use devices. They are essentially boxes, containing all the electronics, with two connectors; one interfaces the engine ECU and the other, a serial or a USB connector, depending on the type of the DPA, interfaces the data acquisition computer. These serial and USB DPA adapters can be seen in Figure 7 and Figure 8, respectively. 


\section{DG Dearborn Group J1939 • $\underset{\text { CAN }}{\text { Sw }}$}

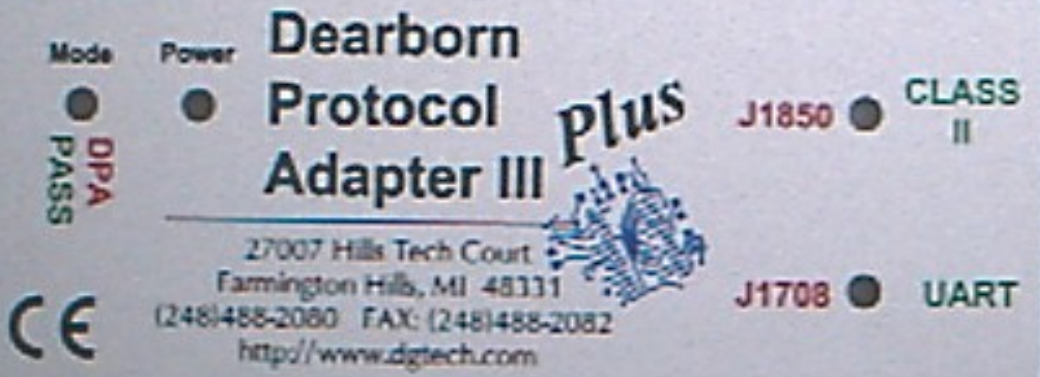

Figure 7 Serial DPA Adapter [2]

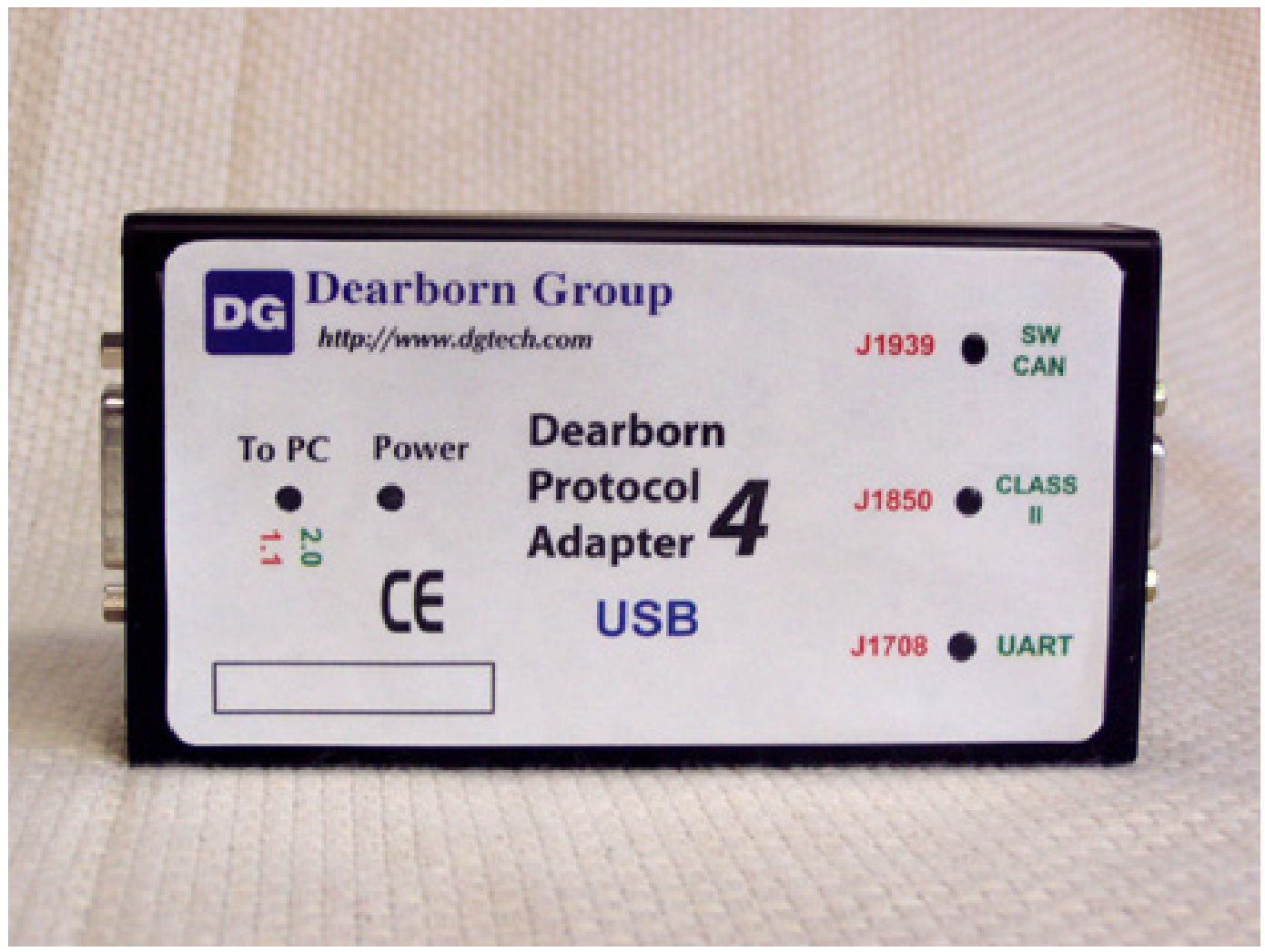

Figure 8 USB DPA Adapter [2] 
Two laptop computers were used to collect the data. One was Toshiba Tecra A3 with Intel Pentium M processor, $1.60 \mathrm{GHz}$ with $504 \mathrm{MB}$ of RAM, and the other was Compaq Presario 2100 with AMD Athlon XP2400+ processor, $1.79 \mathrm{GHz}$ with $704 \mathrm{MB}$ of RAM. Both laptop computers were equipped with one serial RS 232 port and multiple USB ports. Both computers had installed DLM software that is used to log the engine ECU data. With each computer having serial and USB ports, it is possible to collect simultaneously data through multiple DPA adapters per computer. In this study, at most two DPA adapters were connected to one computer, for a maximum total of four adapters used at the same time during a test.

A screen capture of the DLM software user interface can be seen in Figure 9. The most obvious feature of this screen is a window visualizing the streaming ECU data traffic. There are numerous options available to the user, allowing to select which protocol broadcast to collect, to filter or to flag specific messages, or to trigger certain functions upon receiving a specified message. The received data can be saved to a user specified file with the option of a data buffer employment. 


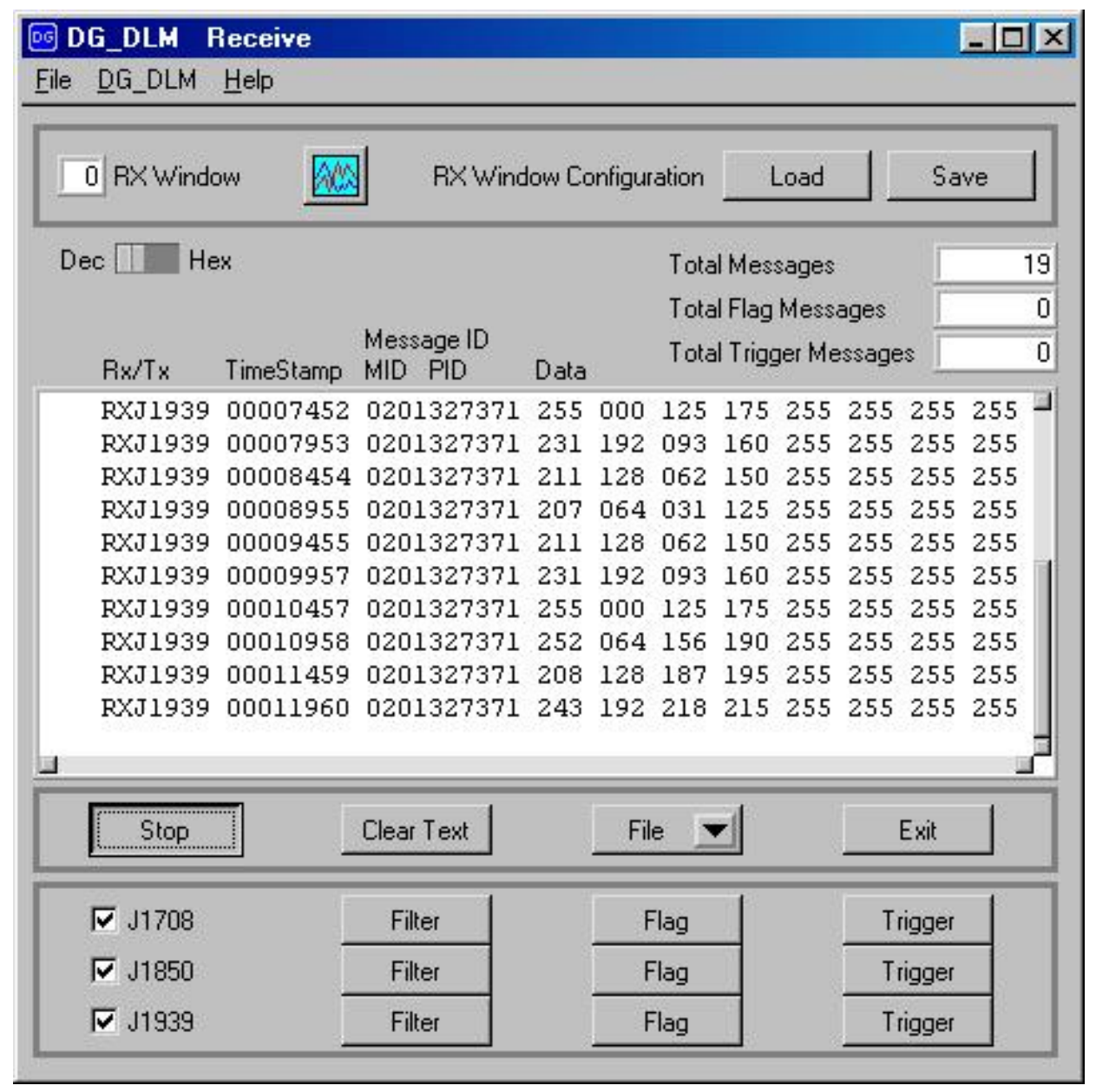

Figure 9 Screen Capture of the DLM software [2]

In order to collect the data with multiple adapters, a branching cable was made to provide a link between the engine ECU, and each one of the used DPA adapters. The connector used on the DLM adapter on the engine ECU communication side is DB 15, a commonly available connector with 15 pins arranged in two rows. The pin function assignment used on the DLM adapter is provided on Dearborn Group's website and is pictured in Table 4 [2]. The actual view of the DB15 connector can be seen in Figure 10, which indicates the pin numbering order. The pins that are dark in this figure denote the pins actually used for the DPA unlike the white pins that were not used for this application. 


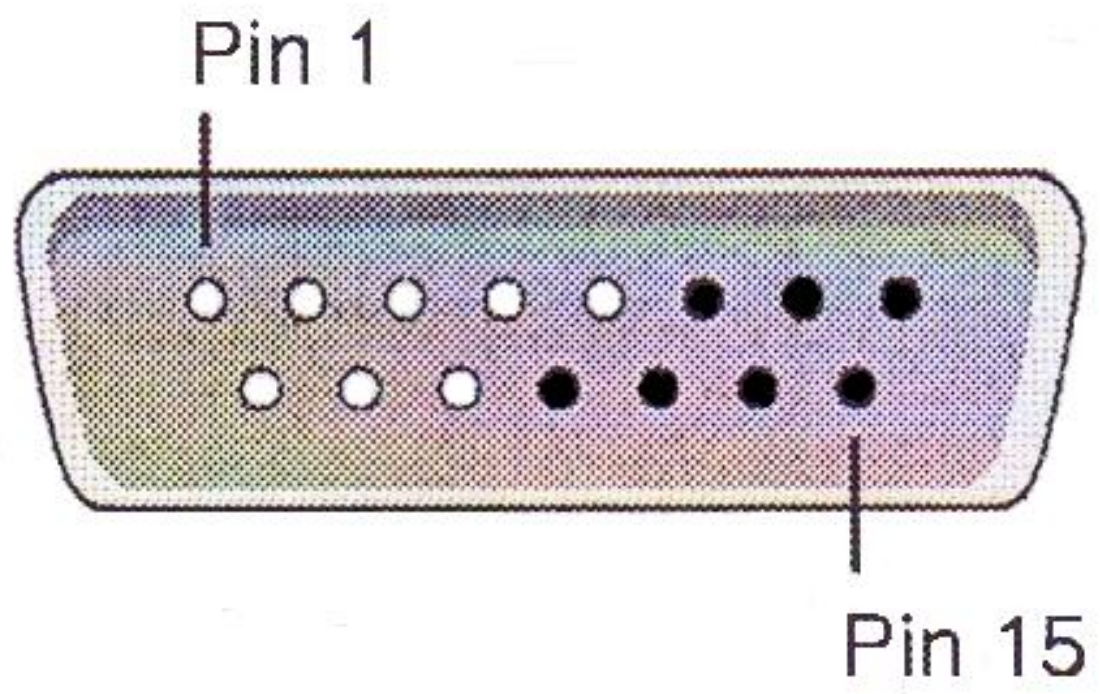

Figure 10 DB15 Connector with Indicated Numbering Order [5]

Table 4 DLM Adapter Pin Out [2]

\begin{tabular}{|l|c|c|}
\hline & $\begin{array}{c}\text { Serial } \\
\text { DPA III+/T }\end{array}$ & $\begin{array}{c}\text { USB } \\
\text { DPA 4 }\end{array}$ \\
\hline Ground & 6 & 6 \\
\hline Power(9-32VDC) & 8 & 8 \\
\hline J1708 - & 14 & 14 \\
\hline J1708 + & 15 & 15 \\
\hline CAN Shield & 7 & 7 \\
\hline CAN Lo & 12 & 12 \\
\hline CAN Hi & 13 & 13 \\
\hline
\end{tabular}

Also, another cable was made to accommodate the connection between the ECU and the DPA adapters. The connection was made directly in the main connector at the engine ECU. The pin-out of the DDC 60 ECU connector is shown in Figure 11. 


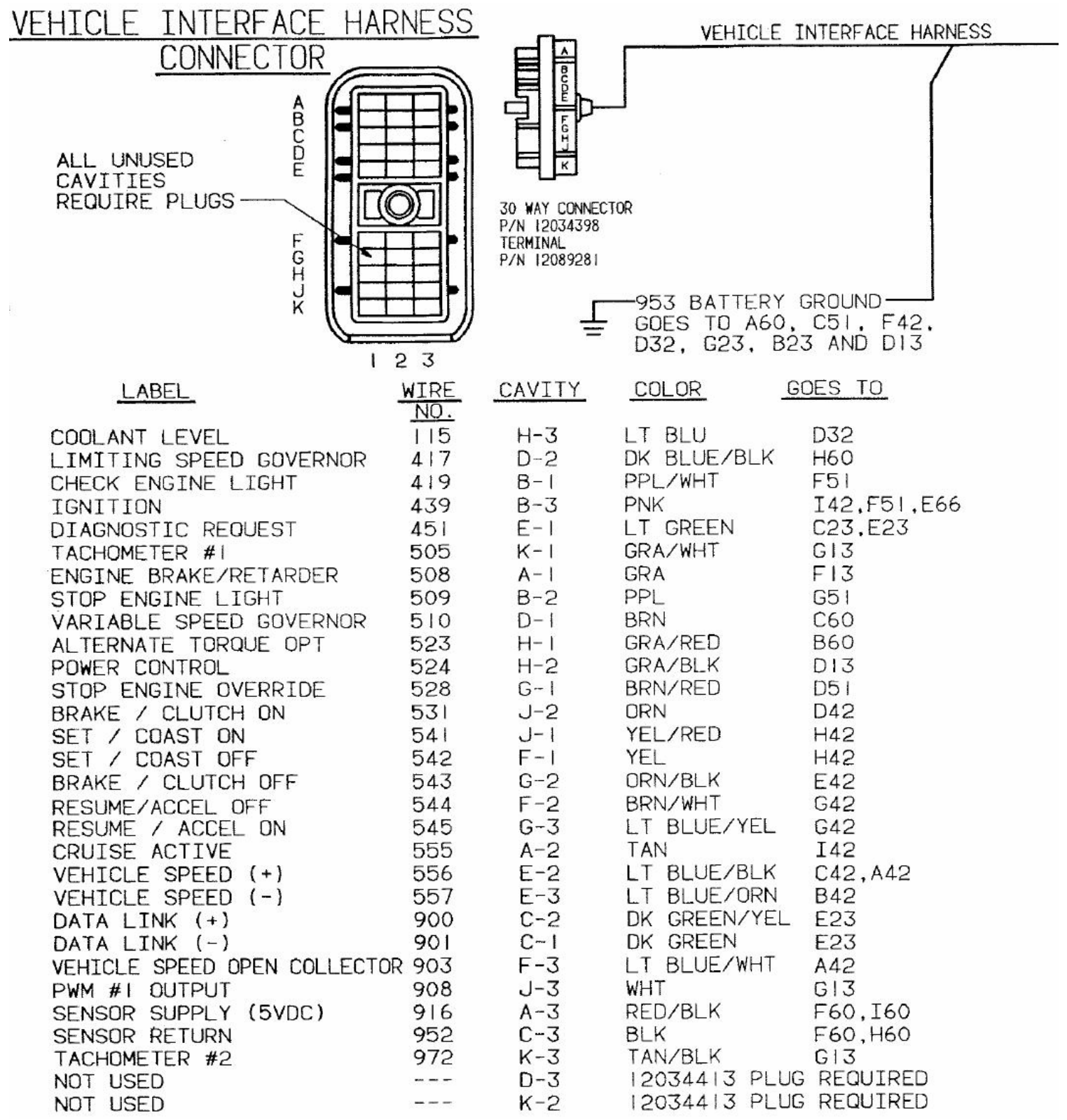

Figure 11 Pin-out of the ECM connector on the 1992 DDC 60 Engine [13 ]

Figure 12 shows two connectors on the 2004 Volvo MD 11 engine ECU. The connectors are marked A and B and although they are physically the same, the pin assignments are different between them. Figure 13 shows the female connector that mates with the male ECU connector. This figure is included to clarify the numbering system of the pins in the connector. Table 5 list the pins used for ECU protocol communication links. 


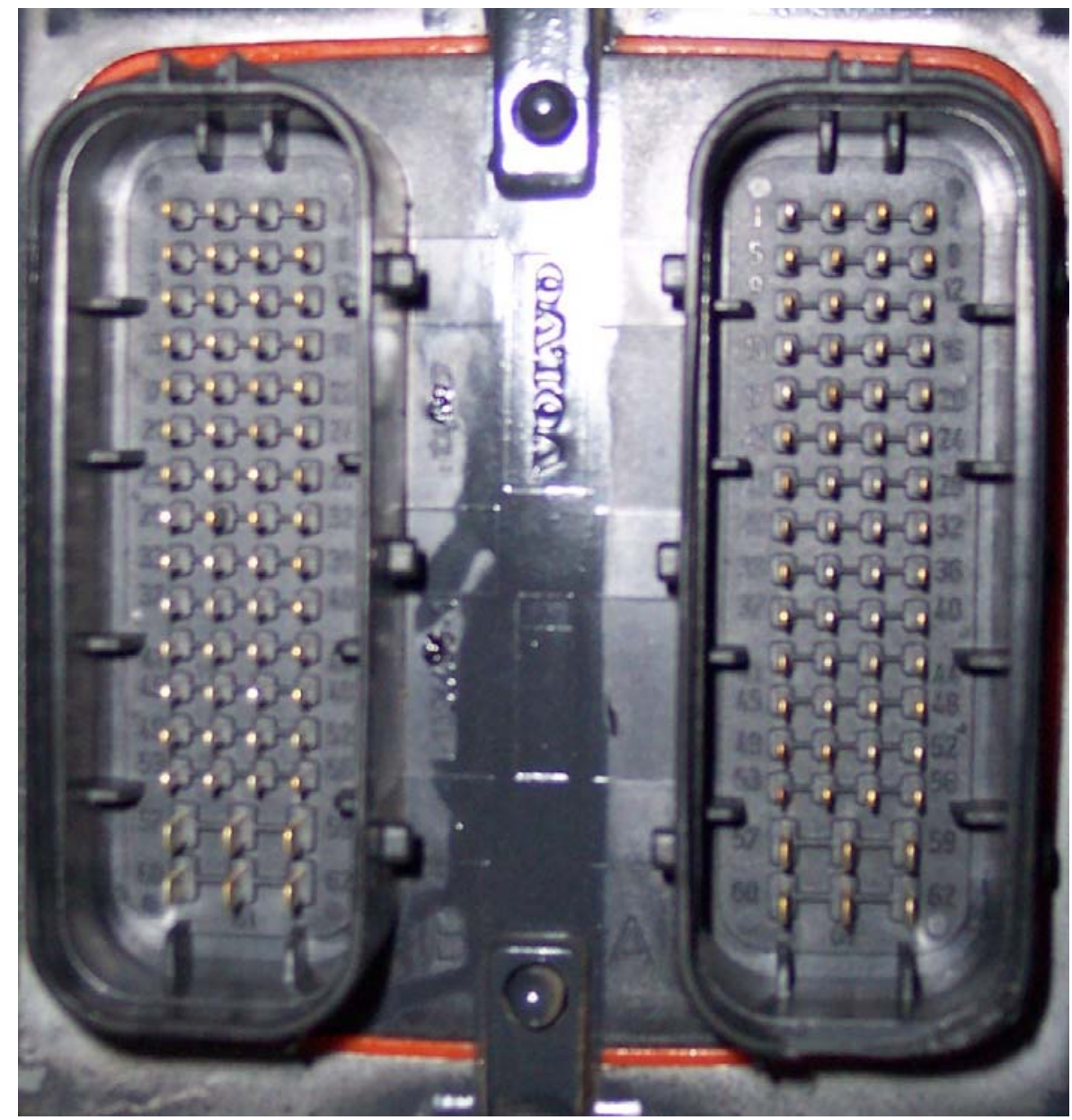

Figure 12 Connectors B (Left) and A (Right) On the Volvo MD 11 Engine ECU

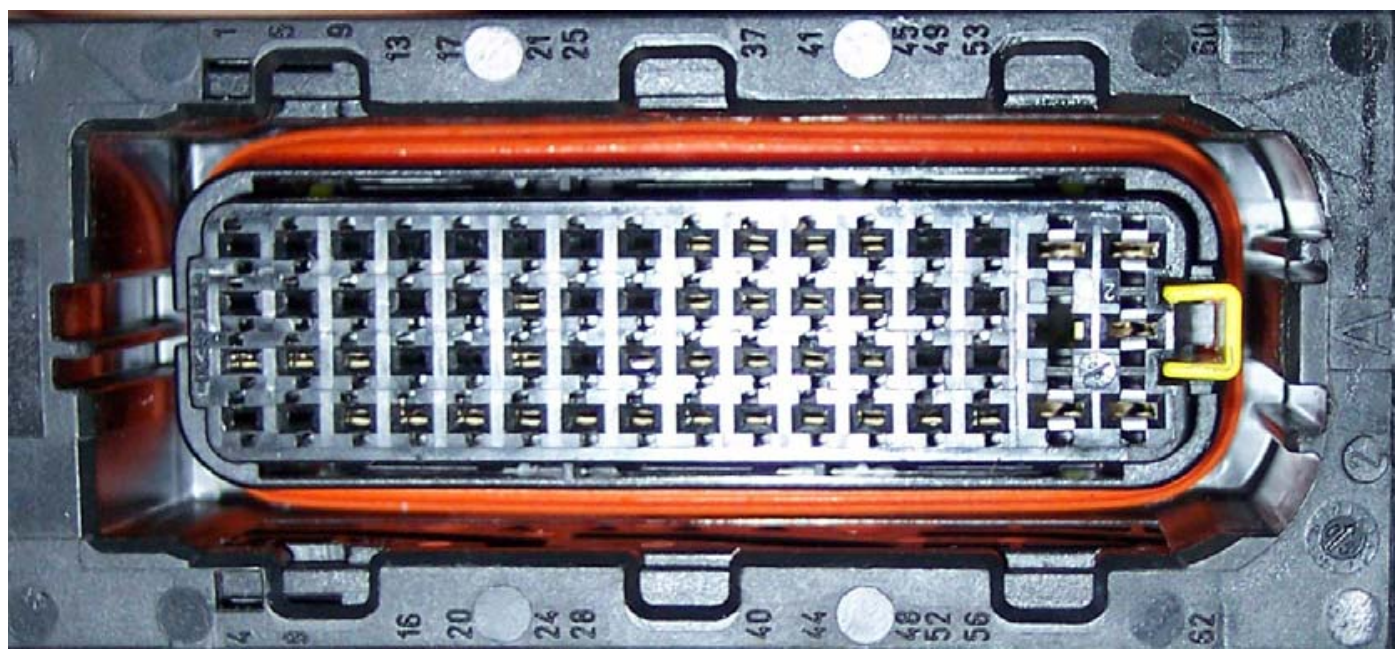

Figure 13 Pin Numbering Pattern of Volvo MD 11 Engine ECU Connectors 
Table 5 Volvo MD 11 ECU Communication Link Pin-Out

\begin{tabular}{|c|c|c|}
\hline & Pin No. & Pin No. \\
\hline & Connector A & Connector B \\
\hline $1708+$ & 34 & N/A \\
\hline $1708-$ & 33 & N/A \\
\hline CANhi & N/A & 51 \\
\hline CANlo & N/A & 55 \\
\hline
\end{tabular}

It should be noted that when connecting to 1939 CAN link on a stand-alone engine, a 60 ohm resistor will most likely need to be connected between CAN Hi and CAN Lo. Detailed information about the application of the resistor can be found in Section 3.2.4.

\subsection{On Road Test Setup}

The on-road testing for this study was conducted in parallel with ongoing in-use emission testing with WVU's MEMS with addition of an extra serial DPA and its dedicated data acquisition laptop computer.

\subsubsection{MEMS Description}

The MEMS system consists of several major components [8]. The first major component is the exhaust flow rate measurement tube that is fitted on a vehicle exhaust pipe. The critical component of this device is an averaging differential pressure flow measurement apparatus mounted inside the tube. The flow rate measurement tube is in addition equipped with the emissions sampling probe, K- type thermocouples and a box containing differential and absolute pressure sensors, valves and supporting electronics. Figure 14 show the flow tube with all the described additional equipment mounted to the tail pipe of a bus. 


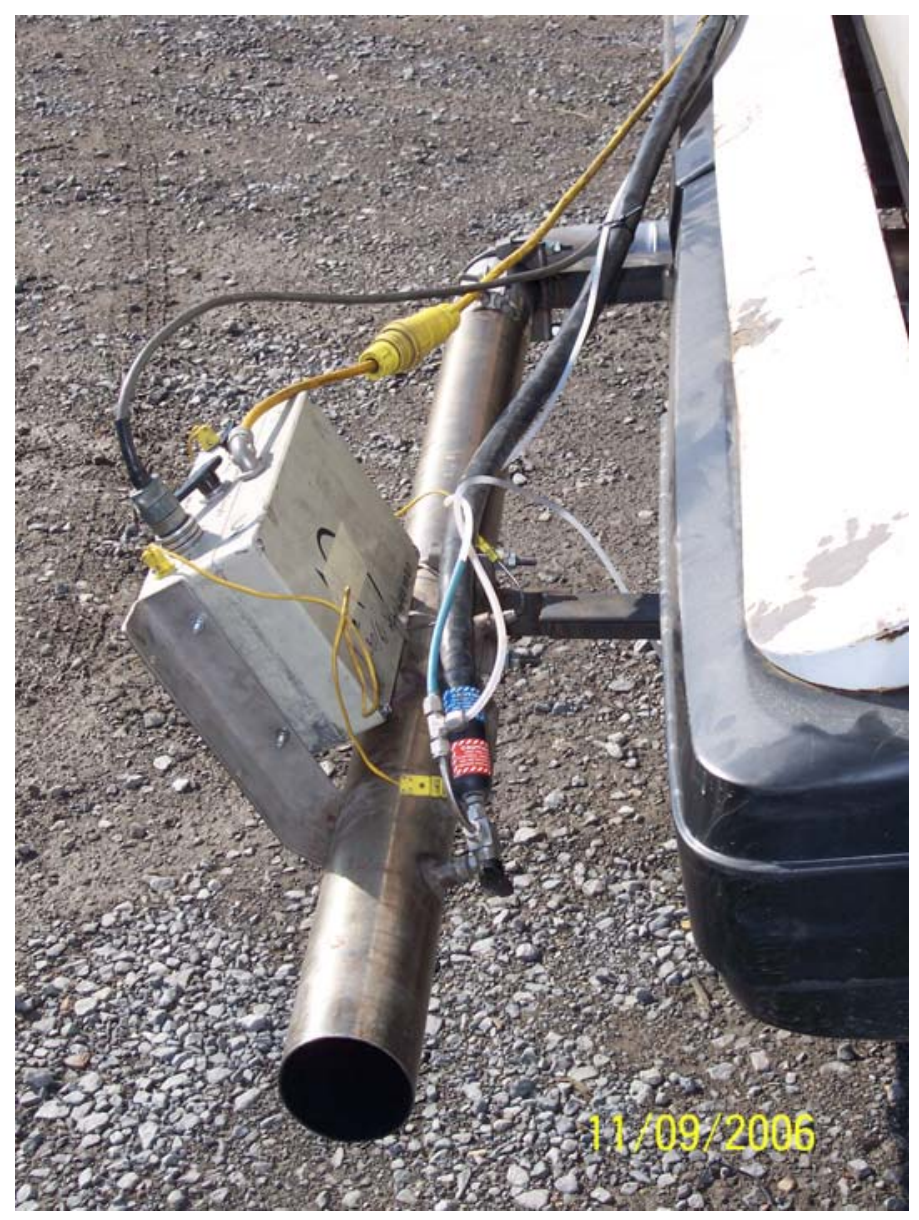

Figure 14 Flow Measurement Tube

The second major component of the MEMS system is the emissions box that houses all the gas analyzers and supporting equipment such as a heated filter, sample pump, sample chiller and temperature controllers. The purpose of the emission box is to draw a sample of an exhaust gas from the sample probe through a heated line, condition the sample and analyze it. MEMS use a Horiba BE-140 non dispersive infra red (NDIR) analyzer for measurement of exhaust $\mathrm{CO}_{2}$ concentration. The exhaust $\mathrm{NOx}$ concentrations are measured by a Horiba MEXA 720 zirconium oxide analyzer and by a Sensors Inc. electrochemical cell analyzer. The instantaneous analyzer measurement values are then reported to the data acquisition system that is housed in the data acquisition (DAQ) box, which is the third major component of the MEMS system. Figure 15 shows the DAQ box side by side to the emission box. On top of the DAQ box (left) is 
seen the laptop computer used for logging data. In the left lower corner of the emission box is visible the opening into which the heated sample line is mounted. The thick cable near the right lower corner is the umbilical cord connecting the emission box to the DAQ box.

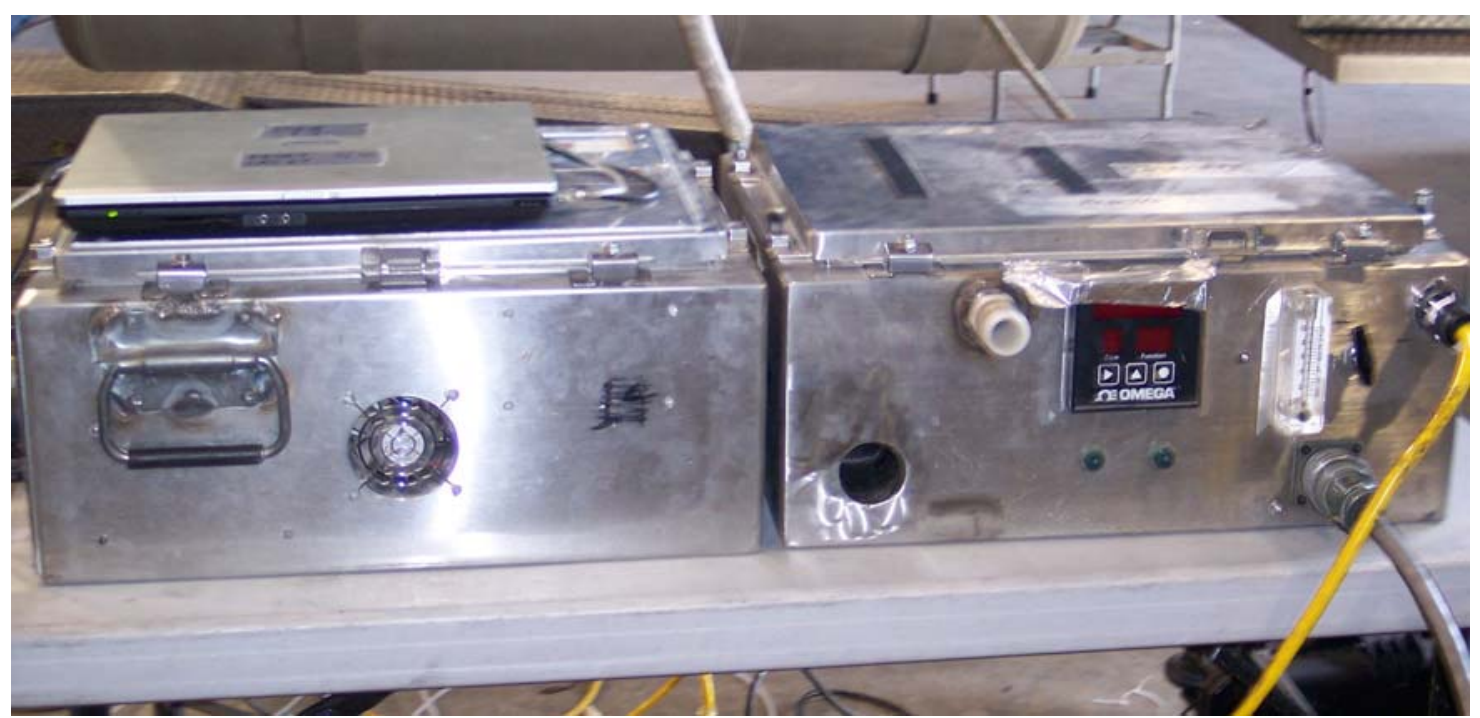

Figure 15 MEMS Data Acquisition (left) and Emission (right) Boxes

\subsubsection{The Test Vehicles and Setup}

Vehicles used for these on road tests were Class-8 tractors and buses. As tractors were frequently tested with the WVU's MEMS testing program, there was a trailer specially equipped and designated for this task. Beside the load that consisted of various concrete blocks, the trailer was outfitted with a booth to house most of the MEMS equipment, and with a power generator to provide the AC power needed to run all the equipment. This setup provided quite a convenience as only the flow tube needed to be fitted onto the actual vehicle, which in most cases involved removal of the exhaust stack and the muffler and fitting the flow tube instead. The tractor was then coupled to the trailer, all the necessary connections, such as the heated line, and data and power cables were made, and the vehicle was ready to be tested. Unlike tractors, other types of vehicles 
such as buses and box trucks had to be equipped with all the equipment and a load on individual basis.

\subsubsection{ECU Connection}

Connecting to the engine ECU is somewhat simpler on a vehicle than on the engine alone as discussed in Section 3.1. Typically, on a vehicle, specific ECU communication connectors, which are standardized by the industry, are already installed by the manufacturer. These connectors are Deutsch 9 pin, Deutsch 6 pin and Deutsch 3 pin. Figure 16, Figure 17 and Figure 18 picture the Deutsch 9, 6 and 3 pin connectors, respectively, showing their pin layout and marking letter assignment. The appropriate pin-out for these connectors is listed in Table 6.
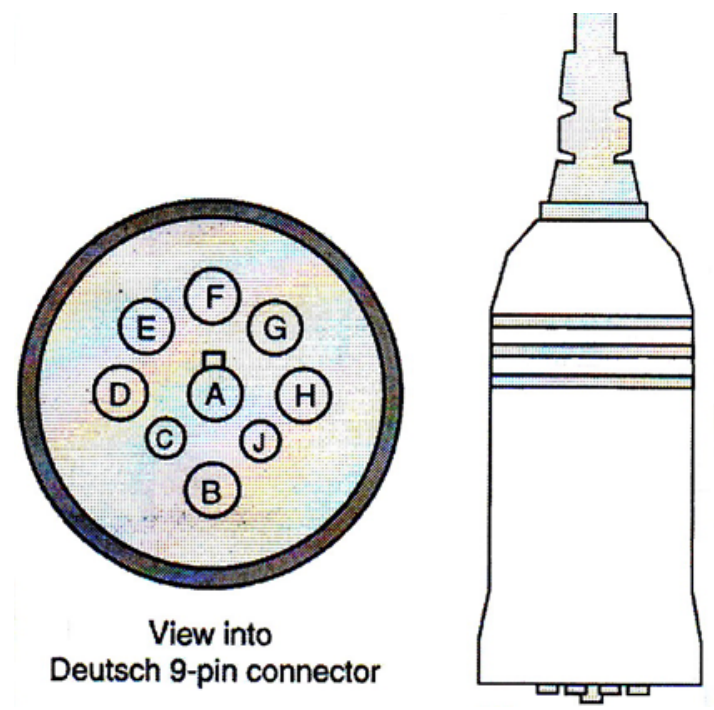

Figure 16 Deutsch 9-Pin Connector - Pin Letter Marking Assignment [6] 

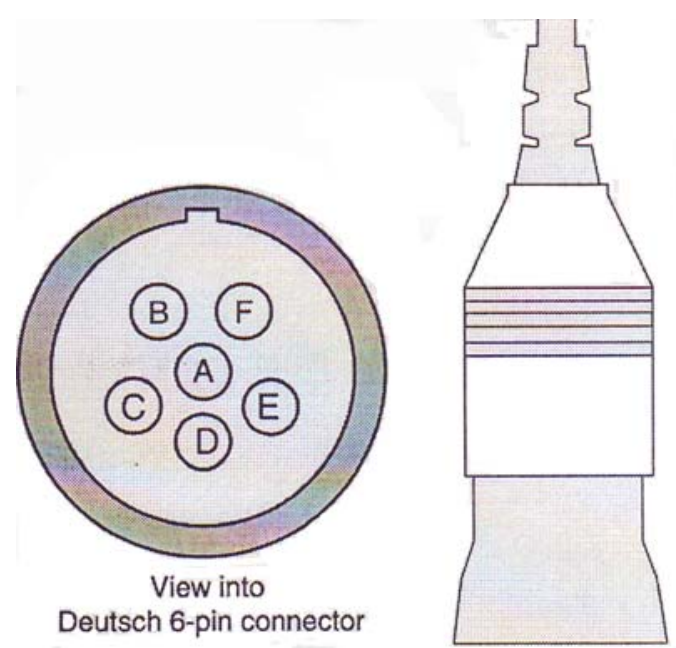

Figure 17 Deutsch 6-Pin Connector - Pin Letter Marking Assignment [5]
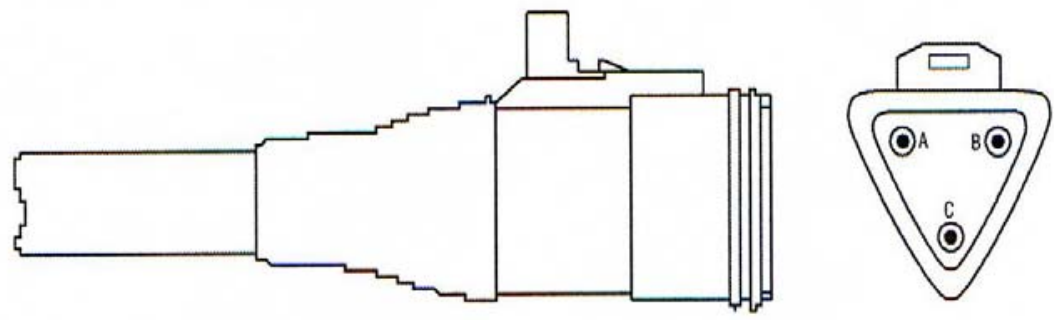

Figure 18 Deutsch 3-Pin Connector - Pin Letter Marking Assignment [7]

Table 6 Pin-out of the Various Deutsch Connectors Used for ECU Communication

\begin{tabular}{|l|c|c|c|}
\hline & $\begin{array}{c}9 \text { Pin } \\
\text { Deutsch }\end{array}$ & $\begin{array}{c}6 \text { Pin } \\
\text { Deutsch }\end{array}$ & $\begin{array}{c}3 \text { Pin } \\
\text { Deutsch }\end{array}$ \\
\hline Ground & A & E & - \\
\hline Power(9-32VDC) & B & C & - \\
\hline J1708 - & G & B & - \\
\hline J1708 + & F & A & - \\
\hline CAN Shield & E & - & C \\
\hline CAN Lo & D & - & B \\
\hline CAN Hi & C & - & A \\
\hline
\end{tabular}

The 6 pin Deutsch only provides J1708 link for J1587 protocol communication and is used mostly with older engines while the 9 pin Deutsch connector usually provides both the J1708 and the J1939 CAN links. However some manufacturers use the 6 or 9 pin 
connectors with only the J1708 link even with their newer engines and complement it with the 3 pin Deutsch that provides the J1939 link. Either of the 6 or 9 pin connectors is usually placed in the cab under the dashboard near the steering column, while the 3 pin connector is usually located in the engine compartment dangling on the wiring harness near the engine ECU. The DPA hardware is then connected to the ECU via a cable with a Deutsch connector on one side and the DB 15 on the other. Also, the same branching cable with multiple DB 15 connectors as described for the laboratory setup in Section 3.1 is used in the on-road test setup to collect the ECU data by multiple devices. During normal in-use emissions testing with MEMS two DPA adapters were used, one was the serial type which was operating with the data acquisition program created at WVU for MEMS specifically and the other was the USB model and was run with the DLM data acquisition software. Sometimes, depending on the engine manufacturer's request, there was some proprietary ECU interacting and data logging equipment used, in addition to the two DPA adapters described above. For this study, one more DPA serial adapter was added with its dedicated laptop computer that would collect ECU data using the DLM software. This additional serial adapter would be collecting exactly the same data that the usually employed USB DPA collects, so the differences between the two obtained data sets could be compared.

\subsubsection{Details on Connecting to J1939 CAN Link}

A CAN consists of a main line called the "back bone" which is a shielded twisted pair of wires terminated at each end by a 120 ohm resistor. The maximum length of the back bone is specified to be 40 meters. To this back bone can be connected up to 30 nodes of maximum 1 meter length with ECUs. The detailed specifications can be found in SAE publication J1939/11. Figure 19 shows the described architecture. With the 120 
ohm terminating resistors connected between $\mathrm{CAN}_{\mathrm{HI}}$ and $\mathrm{CAN}_{\mathrm{LO}}$ conductors at each end of the back bone, each ECU is interfacing a loop with 60 ohm resistance.

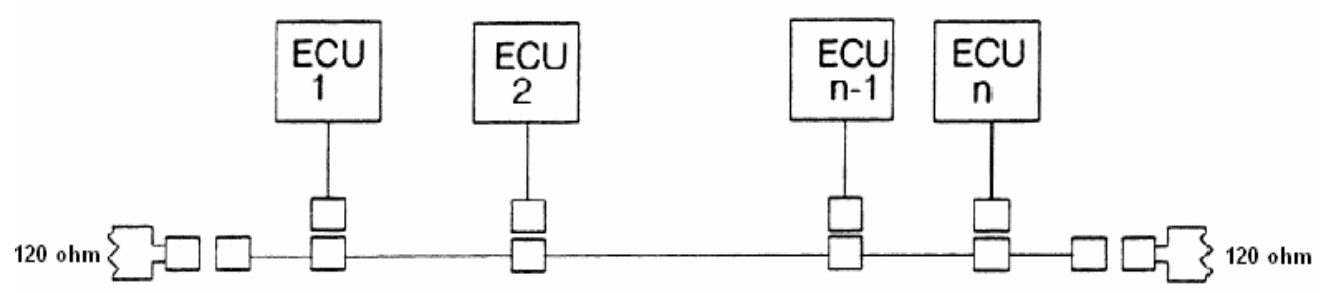

Figure 19 Controller Area Network J1939 Architecture [11]

When connecting to CAN on a vehicle, one is faced with a connector to which to connect the protocol adapter. The problem is that there is not always a complete CAN with correct back bone present in the vehicle and the connector is not always the same. Figure 20 shows the 3 cases of connection configuration possible along with the connectors that can be encountered.

Case 1 indicates a complete CAN with the correct terminating resistors. In this case one will need a Connector A to plug into the harness and there is no need for additional terminating resistors needed. This case can be verified by measuring resistance between $\mathrm{CAN}_{\mathrm{HI}}$ and $\mathrm{CAN}_{\mathrm{LO}}$ pins. This should read around 60 ohms. This resistance should be measured in both directions with the vehicle power shut off.

Case 2 is an incomplete back bone with only one end terminated. One will need a Connector B to plug in to the harness. Verification of this situation by resistance measurement procedure, as in Case 1, will yield reading of 120 ohms. In this case another resistor of 120 ohms will need to be placed between $\mathrm{CAN}_{\mathrm{HI}}$ and $\mathrm{CAN}_{\mathrm{LO}}$.

In Case 3, there is none of the back bone present and one has to connect into the ECU directly by either plugging into Connector A or by picking the pins in the main 
ECU connector. For this case the resistance verification will yield values on the order of kohms and a resistor of 60 ohms will need to be added between $\mathrm{CAN}_{\mathrm{HI}}$ and $\mathrm{CAN}_{\mathrm{LO}}$.

When connecting to the 9 pin Deutsch connector under the dash, any of these 3 cases could apply. The only way to determine, which case is faced, is to measure the resistance as described above. However, a simple way to get the connection to work is to simply attempt all 3 cases by starting with no resistor, then trying 120 ohms and if that still does not work, trying the $60 \mathrm{ohm}$ resistor. Unless there is some other problem, a connection should be established after this procedure.

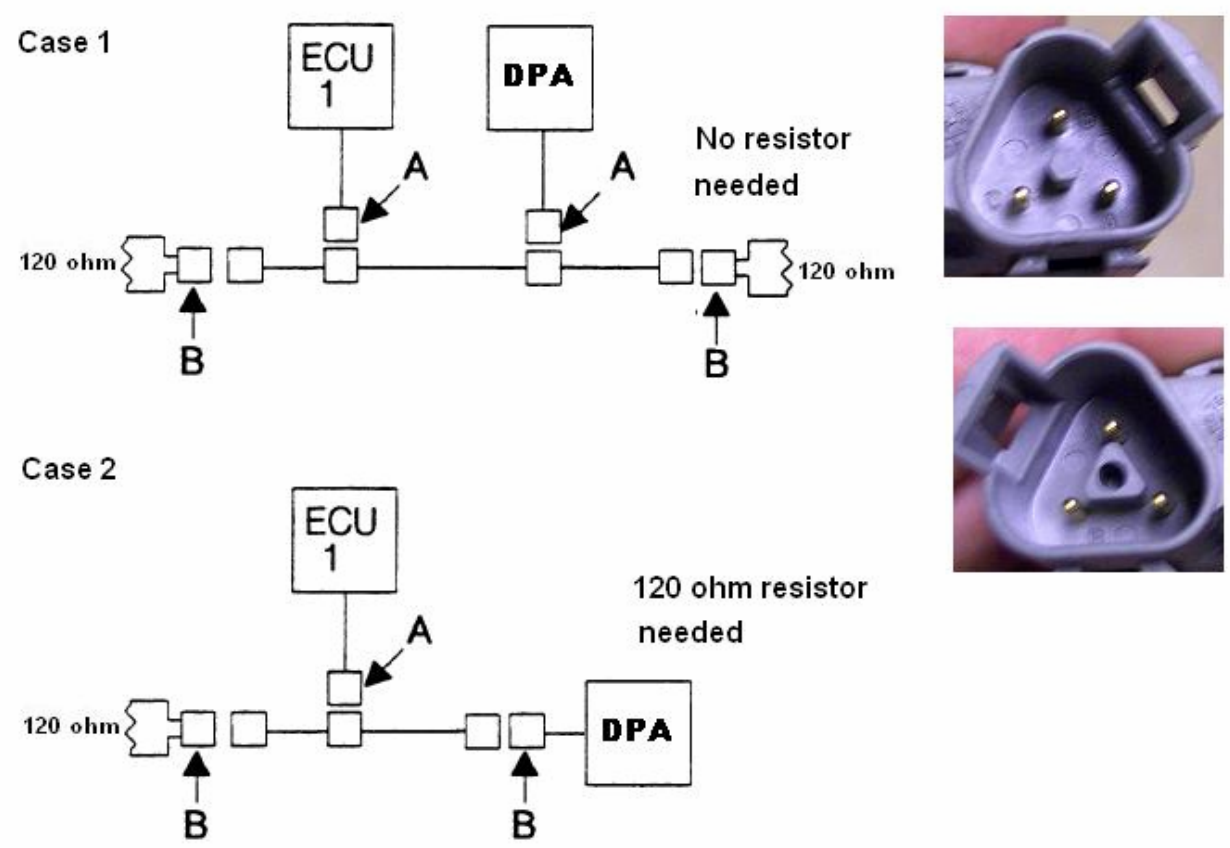

Case 3

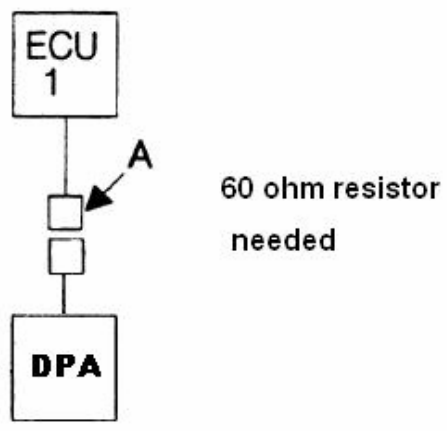

Figure 20 Guide for Connecting DPA to J1939 link 


\section{Methodology}

\subsection{Background}

\subsubsection{Difficulties With On-Road Data Acquisition}

Since the development and verification of the MEMS for over the road testing, there were some updates and changes made in the system. These changes were initiated for various reasons such as requests for additional data by the engine manufacturers or due to encountered problems with component longevity. One of these changes was implementation of a USB DPA adapter instead of the original adapter which communicated with the data acquisition computer via serial RS232 port. The reason for switching to USB DPA adapter was simplification of the data acquisition system. The original MEMS data acquisition system employed a PC based computer that was custom built at WVU from over-the-counter components mostly intended for desktop applications rather than rough condition operation [8]. These components did not cope well with the vibrations encountered when placed on a heavy duty vehicle operating on a road and failed frequently, resulting in time costing delays in testing schedules. A decision was made to rework the data acquisition system to implement notebook computers which are designed to withstand some rough handling with reasonable reliability. This switch to a notebook computer resulted in a limited number of available serial ports. Newer laptop computers usually have only one serial RS232 port, or none at all. Since the only available serial port was used up by another part of the data acquisition system, there were two options of either using two laptop computers or using a USB DPA adapter instead of the serial adapter. The USB DPA adapter was adopted and a single data acquisition computer was chosen and has been in use ever since.

\subsubsection{Description of Error Encountered in MEMS Data}

It became apparent at a later point during MEMS testing, that there was something wrong with the data logged with the DPA adapter and DLM software. Some 
of the tests conducted with MEMS produced emission results that were suspect, and upon closer inspection, there was an apparent time misalignment of the emissions data and the power obtained from the ECU logged data. The DLM ECU data and the MEMS emission data, as collected, were misaligned. One of the reasons for this misalignment is that there was more than one program used to collect data and these were not started at exactly the exact same time. The other cause for the data misalignment was the length of the sampling line. Deskew time causes misalignment, which is then corrected by shifting the data during the data reduction process. In practice, this shift correction was done as follows. The MEMS data were reduced with an arbitrary shift and the resulting data were inspected for any remaining misalignment between the emission data an engine power data. This was done by viewing plots of emission mass rate and engine power versus time. The traces of the emission constituent mass rates are very similar to the traces of engine power. In other words, peaks and valleys in engine power output should correspond to peaks and valleys of mass rate of emissions with sufficient similarity to allow for alignment. This similarity is very pronounced with the $\mathrm{CO}_{2}$ emission rate, but not as much with the NOx emission rate. NOx formation depends greatly on a combustion temperature, therefore its mass rate has strong dependency on engine torque rather then engine speed. Nonetheless, the NOx mass rate still matches the power sufficiently to do the alignment. The time difference was then determined from these plots and the obtained time shift was then inserted into the data reduction program when the data were reduced again. With the data correctly aligned, the correct brake specific emissions could be then calculated. Figure 21 demonstrates a set of engine power and $\mathrm{CO}_{2}$ mass rate data in need of alignment, since there is a slip of about 3 second between these two data sets. Figure 22 shows the same two sets of data that were reduced again with the time shift corrected. The same time shift correction was also done with the NOx 
data from both the MEXA and electrochemical analyzers of the MEMS. Normally, with a healthy set of test data, this alignment of emissions with power is consistent throughout the whole duration of the test.

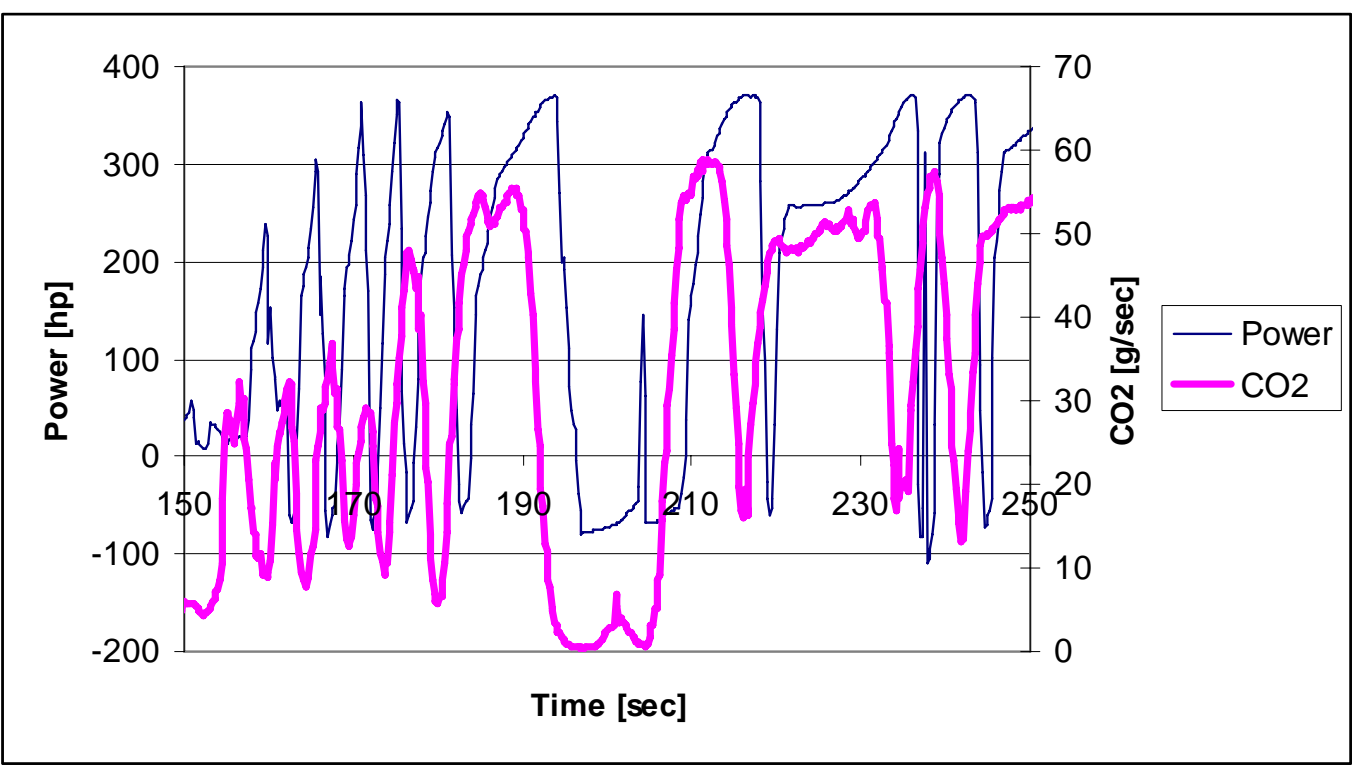

Figure 21 Misaligned Engine Power and Emission Data

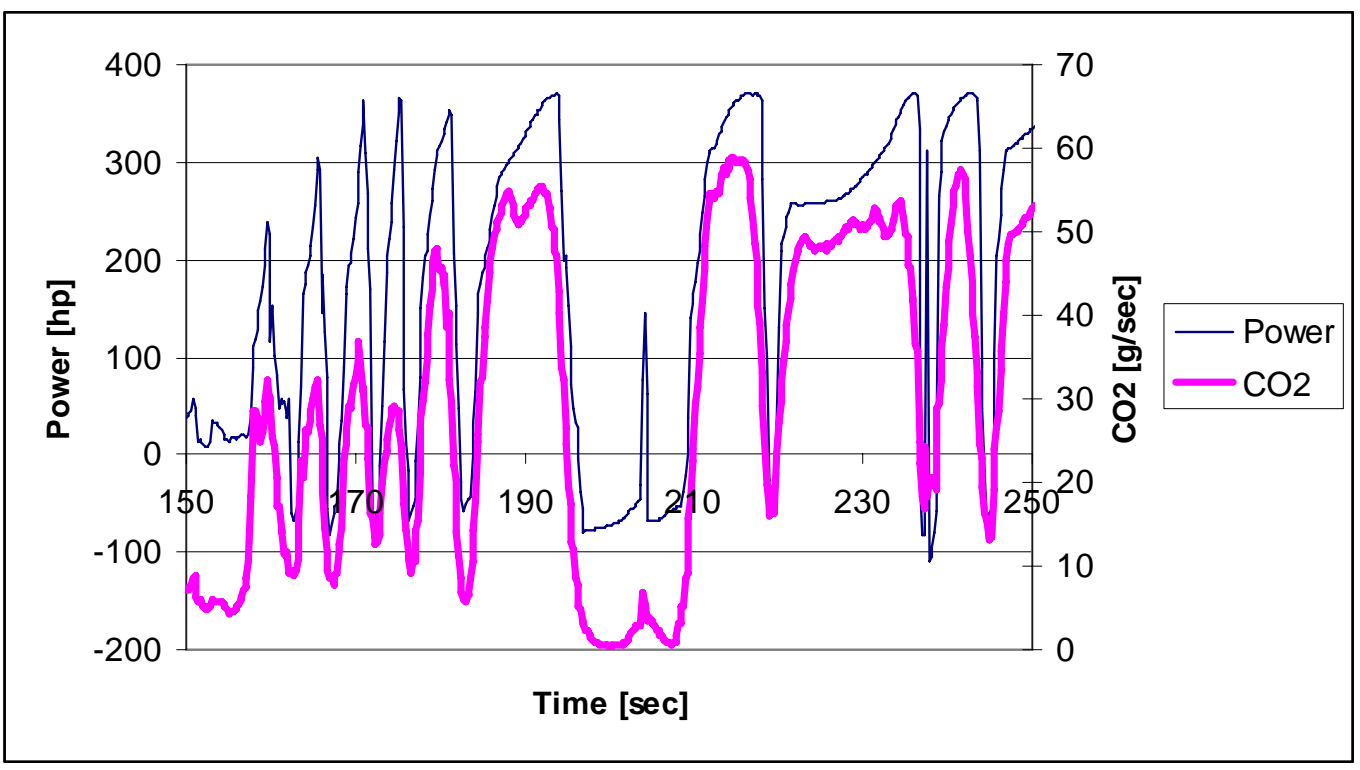

Figure 22 Aligned Engine Power and Emission Data

The problem was that some of the tests produced data that, although aligned at the beginning of the test, showed a misalignment further into the test. Figure 23 and Figure 24 demonstrate this problem. These figures show plots of power and a mass 
emissions rate of $\mathrm{CO}_{2}$ from an older MEMS test that produced suspicious values of brake specific emissions. Closer inspection revealed that the power data set seems to be of different length than the emissions data set.

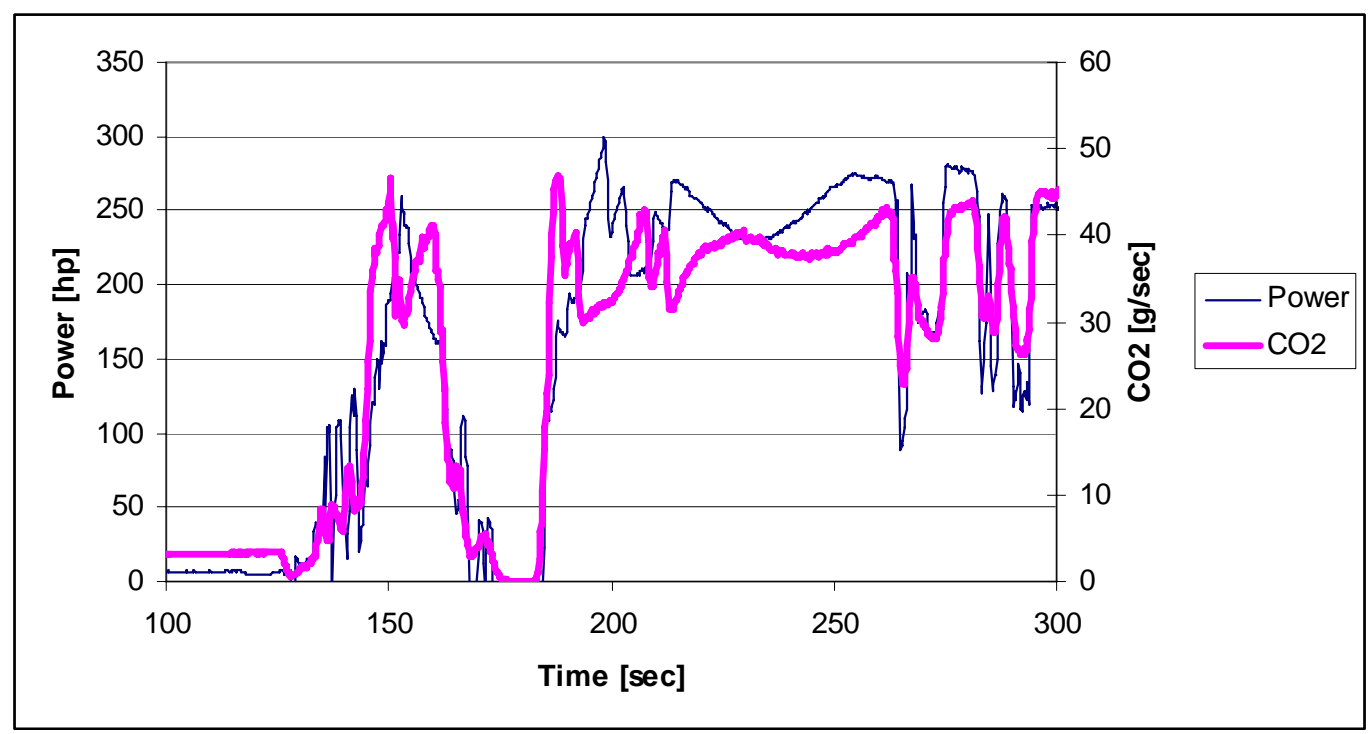

Figure 23 MEMS Test Data Aligned at the Beginning of the Test

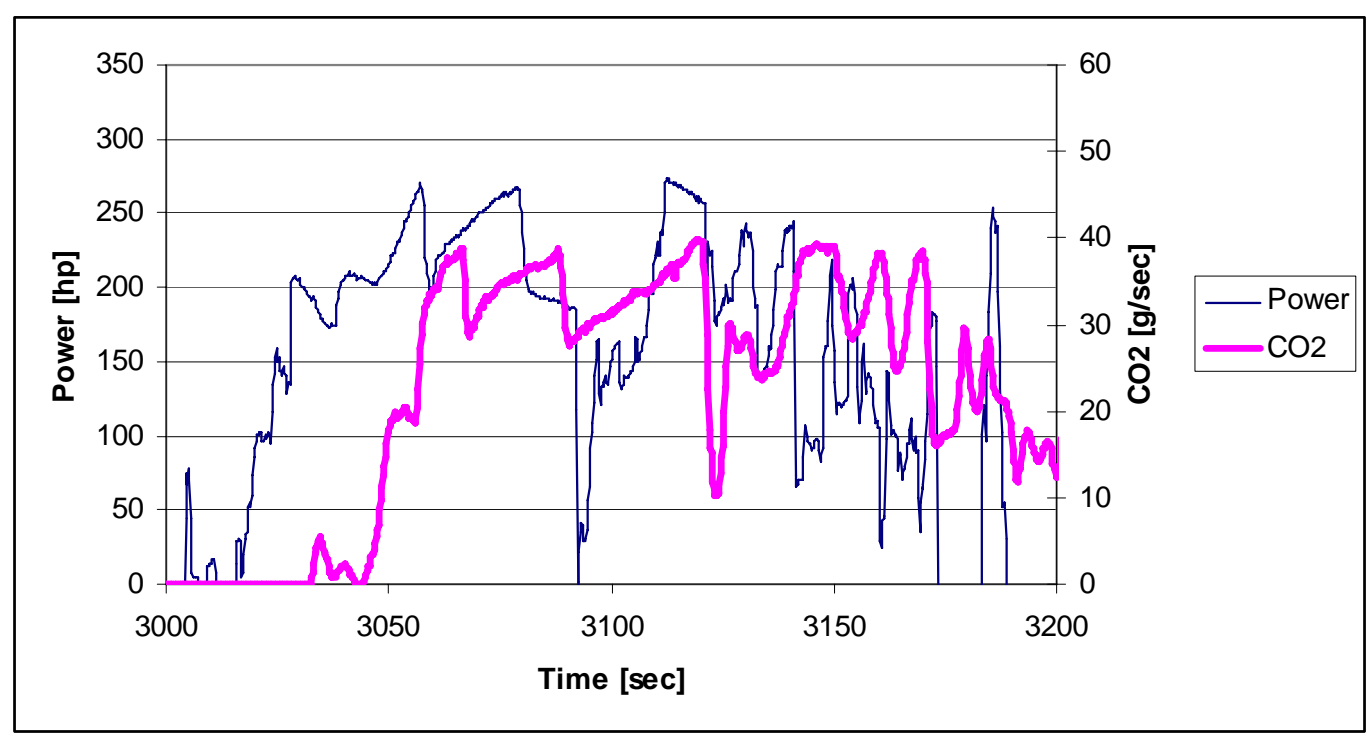

Figure 24 MEMS Test Data Showing Discrepancy at the End of the Test

Even though both data sets were aligned at the beginning of the test, as can be seen in Figure 23, they are not aligned throughout the whole test, and by the end of the test the misalignment is quite significant as depicted in Figure 24. This misalignment makes it impossible to produce correct values of brake specific emissions; hence, it is the subject 
of this study. Also noteworthy is the inconsistency in the shape of plots of the power and the mass emissions rate of $\mathrm{CO}_{2}$ in the error-affected tests as seen in Figure 23. These, although still somewhat similar in major features, have significant differences in the shape details. In comparison, the same plots match each other much better in details, when obtained from a test that does not have error in the DLM data, such as the data plots seen in Figure 22.

\subsection{Error Source Identification}

The first part of this study was conducted in the laboratory and the objective was to determine if and under what circumstances does the DPA/ DLM equipment collect erroneous data. In these experiments the engines were run on a dynamometer. The engine and dynamometer controller works on a system where the desired speed is set by the dynamometer and the desired torque is achieved by changing the fueling rate on the engine. This control method creates engine operation where the engine speed is very accurate and consistent with the desired speed during the transient cycle. This allows all the test runs to be very repeatable and comparable to each other. During these tests, the engine ECU data were collected using two laptop computers and several DLM adapters. Each computer had one serial RS 232 port available in addition to USB ports. This allowed each computer to collect DLM data with at least one serial DPA adapter and one or more USB DPA adapters at the same time depending on how many USB ports were available on the computer. For this study however, a maximum of two adapters were connected to each laptop at one time, one serial and one USB. Many different combinations of hardware were tested to pinpoint the conditions and the cause of error occurrence. To single out the cause of error the following testing variables were introduced: 
1. Numerous DPA adapters were tested to eliminate the possibility of any particular unit being defective and thus causing this error.

2. Different DPA units were cross tested with each computer to identify possible computer incompatibility with any of the adapters.

3. On some tests, the computers were collecting data from two adapters each and on others from one adapter only, to see the possibility of processing power limits of the computers causing the error.

4. One or two data collecting computers were used to eliminate the unlikely possibility of any interference between the two data acquisition systems.

5. The DLM data acquisition software has an option of using a data buffer which was turned on for some tests and off for others to eliminate the possibility of any influence of this function.

6. Two different engines of different year and manufacturer were used.

7. Data broadcasted by two different protocols, the 1587 and 1939 were collected.

\subsection{Error Correction}

The first part of the study established the source of the DLM collected ECU data to be exclusively linked to USB DPA adapter. The next step was to evaluate the projection of this problem onto the on-road MEMS testing accuracy and if possible, to find a method of correcting and post processing the data collected during MEMS testing. The focus is on test data that uses the DLM collected engine data to evaluate the brake specific emissions. It should be noted that for every engine manufacturer, there is a different procedure within the MEMS data reduction software to obtain data necessary for evaluation of the power. This is driven by different protocols followed by different manufacturers. During any given MEMS test there were always two DPA adapters being 
used for collecting ECU data. One DPA adapter was set to collect all possible messages without any restriction. The objective was to collect a broad range of information on engine operation during the test, which would allow for troubleshooting, should the engine malfunction or should the emission test results be unusual. The drawback of collecting all the data is the limited rate at which each parameter can be logged.

However, for the sake of accuracy of the measurement results, it was desirable to collect the parameters determining engine power used to get brake specific emissions (engine torque and speed) at the rate of $5 \mathrm{~Hz}$. Thus, the second DPA applied a filter, which was set just for these parameters to be collected at a high rate. There was normally one serial DPA that collected ECU data using software developed at WVU as a part of the MEMS system and one USB DPA that collected data using the Dearborn DLM software. As already mentioned, each manufacturer’s engine brake specific emissions test data were obtained and reduced using different protocol. Of the six SHDDE manufacturers whose engines were tested with with MEMS (Cummins, Caterpillar, DDC, Mack, Volvo and International), only two manufacturer's engine emissions results were affected by the ECU data time stamp errors. These manufacturers will be referred to as Manufacturer A and Manufacturer B. The reduction program, in the cases these two manufacturers' engine tests, uses torque values from the filtered USB DPA (erroneous) data and the engine speed values come from the unfiltered serial DPA (correct) data. This combination of data with both correct and incorrect timestamps produces power data that when plotted, not only appear short but also reveal discrepancies in the shape. These changes in the shapes of the plotted power data cause difficulties with data alignment as was already demonstrated in Figure 23.

At the time of this testing, there were few remaining vehicles scheduled for in-use testing under WVU contract with the manufacturers. In particular, there were 6 vehicles 
powered by Manufacturer A engines and one vehicle powered by a Manufacturer B engine remaining in in-use testing schedule. These vehicles would be used for obtaining additional test data. As already mentioned, during testing these vehicles, an additional computer with a serial DPA adapter was added to the equipment normally used during standard MEMS testing. This serial DPA adapter would collect exactly the same data as the USB adapter. These data collected with the serial adapter would be assumed as a correct standard to which the erroneous USB data would be compared. The evaluation of correction was done in a following manner. The emission tests were reduced using the customary USB DPA - DLM data as it was a common practice during ordinary MEMS testing. Then there was a correction made to the USB DPA - DLM data files and the test data were reduced again using these new corrected USB DPA - DLM files. Finally the tests were also reduced using the additional serial DPA - DLM files. The resulting brake specific emissions from each of these three data sets were compared to evaluate the correction procedure.

From the data previously collected in the laboratory, it was apparent that the error is caused by incorrect timestamps associated with the data packets collected by the USB DPA. The USB DPA - DLM data appear to have the timestamps assigned with a slower timer. When USB DLM data are compared to serial DLM data collected from the same test, the USB data appear shorter, although there are no missing data points. Profiles of the graphs are the same but the USB data appear to have a shrunken time scale as opposed to the serial data plot. As this time shrinkage appears to be nearly constant during the test, the proposed correction is to linearly stretch the time data associated with the USB DPA collected ECU data to correct length. This suggests that in practice every time point should be multiplied with a certain number that will effectively stretch the time scale of these data points to the desired correct length. To determine this 
multiplication factor, the incorrect USB DPA - DLM data must be compared and matched with some independent, but relevant measurement. The emissions data lend itself for such a comparison. Since the engine power is generated by combustion of a hydrocarbon fuel, it is directly proportional to mass rate of carbon dioxide emissions. The acquisition of emission mass rate of $\mathrm{CO}_{2}$ consists of measurements of raw exhaust concentration of $\mathrm{CO}_{2}$ and the exhaust flow rate. These measurements are independent of the data collected from the ECU; therefore, the $\mathrm{CO}_{2}$ emission mass rate data set was selected as a comparison for the power data set. The engine power and emission mass rate $\mathrm{CO}_{2}$ data sets, when plotted, in fact provide very close resemblance to each other and allow for determining the amount of discrepancy between the emissions and ECU data in terms of time. Using a table processor, such as Microsoft Excel, both data sets were plotted on top of each other although with separate sets of data for time axis. The time data set related to the ECU obtained power data was then stretched until it matched the $\mathrm{CO}_{2}$ mass rate data in respect of aligning the peaks and valleys of both plot traces. The stretch itself was realized by multiplying each time point by a constant number. The selection of the correct amount of stretch was simply done by visual inspection of both traces and confirmation of their correct alignment throughout the whole test. In practice, this was done by visually aligning the plot traces near the beginning of the test (using the first significant align-able feature) by means of shifting the data sets and then aligning the traces at the end by means of applying appropriate stretching factor. This stretching, however, misaligned again the data at the beginning of the test so this procedure was repeated for a few iterations until the correct stretch factor was obtained with the alignment settled over the whole test. The assessment of alignment was done purely on visual basis. 
Now that the correction factor was obtained, it was applied to correct the original

DLM file to be used in the reduction process of the MEMS data. The format of DLM file can be seen in Figure 25.

H RXJ1939, 2748600, 18ffe000, 99, 16, 9b, 7d, 40, a1, 8, 0
H RXJ1939, 2748800, 18ffe000, ae, 16, 94, 7d, 72, 39, 3e, 90
H RXJ1939, 2749000, 18ffe000, bc, 16, 8c, 7d, 12, 40, fd, 80
H RXJ1939, 2749200, 18ffe000, a3, 16, 8c, 7d, c4, 13, 2, 52
H RXJ1939, 2749400, 18ffe000, c3, 16, 8c, 7d, 34, f4, f3, d4
H RXJ1939, 2749600, 18ffe000, a8, 16, 8f, 7d, 8d, 6, 81, 4c
H RXJ1939, 2749800, 18ffe000, 93, 16, 8b, 7d, 50, 41, 62, 56
H RXJ1939, 2749999, 18ffe000, 96, 16, 8e, 7d, f7, e7, 6e, 34
H RXJ1939, 2750200, 18ffe000, 9b, 16, 8d, 7d, d5, 41, 72, d0
H RXJ1939, 2750399, 18ffe000, b3, 16, 90, 7d, 38, 6e, fb, 95
H RXJ1939, 2750599, 18ffe000, c1, 16, 92, 7d, 40, 41, 4a, 10
H RXJ1939, 2750799, 18ffe000, a5, 16, 90, 7d, c9, ef, 50, e5
H RXJ1939, 2750999, 18ffe000, 93, 16, 92, 7d, 10, 34, b3, d2

Figure 25 Example of Data in a DLM File

The second column number of the DLM file is a timestamp associated with the message to the right of it. The number is in a decimal format and represents time in milliseconds. This time is not absolute with respect to the start of the test, in other words, the first timestamp of the test is not zero, and it is just a number that follows the timestamps of previously collected ECU messages. The difference of the timestamps of any two lines of the DLM file gives their time differential in milliseconds. Obtaining the absolute timing of the DLM data with respect of beginning of the collection is simply a matter of subtracting the timestamp of the first line of the file from all the following timestamps. This procedure, however, is not important for this correction method as it is taken care of in the MEMS reduction software itself. To correct the timing of the DLM file, every timestamp of the file being corrected was simply multiplied by an appropriate correction factor obtained by a method already mentioned. The timestamps of the DLM file are in integer format and so the corrected timestamps were also rounded to retain this format. This rounding poses no problems with accuracy as the timestamp’s unit is a millisecond. 
Any rounding is insignificant since MEMS emission data are collected in intervals of 200 milliseconds. This correction was for the most part done using a Microsoft Excel spreadsheet. The DLM file was opened as a Comma Separated Value (CSV) file, then the second column containing the timestamps was multiplied with the correction factor, rounded to an integer and the file was then saved in the same CSV format. This method worked well for all the files collected with tests of Manufacturer A engine powered vehicles. However it did not work for files collected from Manufacturer B engine equipped vehicle test. The reason is that larger amount of messages was selected and filtered during the acquisition of ECU data from the Manufacturer B engine and the resulting DLM files are just simply too long to be opened with the 2002 Microsoft Excel software. To correct these long DLM files, a computer program had to be written. This

program was done in Matlab and is included in Appendix A. To make this program work, the original DLM's file extension “dgd” has to be manually changed to “dat,” before processing it. After the file is processed, it is saved with file extension "txt," which then has to be manually changed back to "dgd.” It should be noted that this program, although it works well, is very slow at completing its task. It is due to Matlab’s difficulty with dealing with files that contain decimal numbers, hexadecimal numbers and characters at the same time. Because of that, the algorithms of this program are not very efficient and cause the process to take long time. If an extensive amount of DLM data correction work was to be done, it would be advisable to write a more efficient program in a different programming language in order to speed up the process.

\subsection{Evaluation of Accuracy of the Corrected Data}

The resulting corrected DLM files were used to reduce the MEMS emissions data just as any ordinary DLM file. The corrected DLM files stayed in the same format and 
the MEMS reduction software digested it as it was never tampered with. The same emission data were reduced using the USB, the corrected USB and the serial DLM files. The resulting horsepower and brake specific emissions data were then compared to each other with the serial data being considered as a correct standard, since in previous testing and comparison with a dynamometer it was determined that the serial data are in fact correct with respect to time. 


\section{Experiment Results}

The objectives of this study were to examine and correct timing errors of ECU data, which were collected from heavy duty diesel engines with the Dearborn Group DPA adapter and DLM software. The first part of this study was designed to identify under what conditions or hardware combination setups did the error occur. The results of this study were then used to create a post processing procedure to correct MEMS data.

\subsection{Identification of the Error}

In these experiments the primary objectives were to find the nature of errors associated with the DLM data and under what conditions were they generated. A whole scheme of experimental combinations of hardware was created to pinpoint the source of this problem with timing of the collected ECU data. The detail of this testing procedure is described in the Section 4.2. There were seven DPA adapters used for testing with an engine dynamometer using a 1992 DDC series 60 engine with ECU broadcasting J1587 protocol data only. There were 36 FTP cycles run with this engine and data were collected from the dynamometer laboratory and the ECU using the DPA adapters and DLM software. The data collected by the laboratory were compared to the ECU data by means of visually inspecting plots of the engine speed. In every single test, the data collected with the USB DPA adapter exhibited significant timing error while the serial data were always in agreement with the laboratory data with very little discrepancy appearing occasionally. This minor discrepancy was always on order of about 0.5 second difference for the 20 minute test and occurred only in four of the tests. The rest of the tests showed the serial data being correct within the boundaries of accuracy of visual inspection 
To confirm these results, this testing procedure was repeated using a 2004 Volvo MD11 heavy duty diesel engine on a dynamometer while collecting ECU data on J1939 CAN link. These tests would eliminate the potential of the error being associated only with one protocol or engine manufacturer. There were 15 FTP cycles run with this configuration collecting data again with the lab and with serial and USB DPA adapters and DLM software. Again, there were multiple adapters tested, in this case only five (three serial DPA and two USB DPAs) as the equipment was shared with ongoing MEMS testing program and only five DPAs were available at the time. Test hardware configurations and software settings were changed just like in the previous procedure. The comparison of engine speed showed from these tests (J1939 protocol) showed consistency with the first set of tests (J1587 protocol) in the fact that every single time the USB adapter produced erroneous data while all the serial data were correct. The serial DPA data from all 15 tests were correct, again, within the limits of visual inspection. The timing error produced by the USB DPA adapter was much larger with the J1939 data then with the J1587 data. The time slip of the USB DPA J1587 data was typically 4-5 seconds while the J1939 USB DPA data showed errors typically of 14-20 seconds per 20 minute FTP test. The higher amount of time slip with the J1939 data is most likely due to higher rate of data transfer on that link. Notably, each adapter tends to collect data with specific amount of time stamp error in all tests. However, there are exceptions to this trend. Appendix D contains tables that summarize the amount of error for each FTP test during which the J1939 data were collected. One table lists all the errors from one USB DPA, which produced errors roughly of 20 seconds in all tests but one occasion, in which the error was 14.4 seconds. Similarly, as listed in the next table, the other USB DPA produced consistent errors of roughly 14 seconds with the exception of one test, in which the error was only 3.2 seconds. 
Figure 26 shows a sample of data collected during an FTP cycle with the DDC 60 engine. It is a plot of engine speed data collected simultaneously by the laboratory's dynamometer speed sensor, serial DPA and USB DPA adapters connected to J1708/J1587 link. All three data sets were aligned at the beginning of the test and the USB DPA shows error of about five seconds.

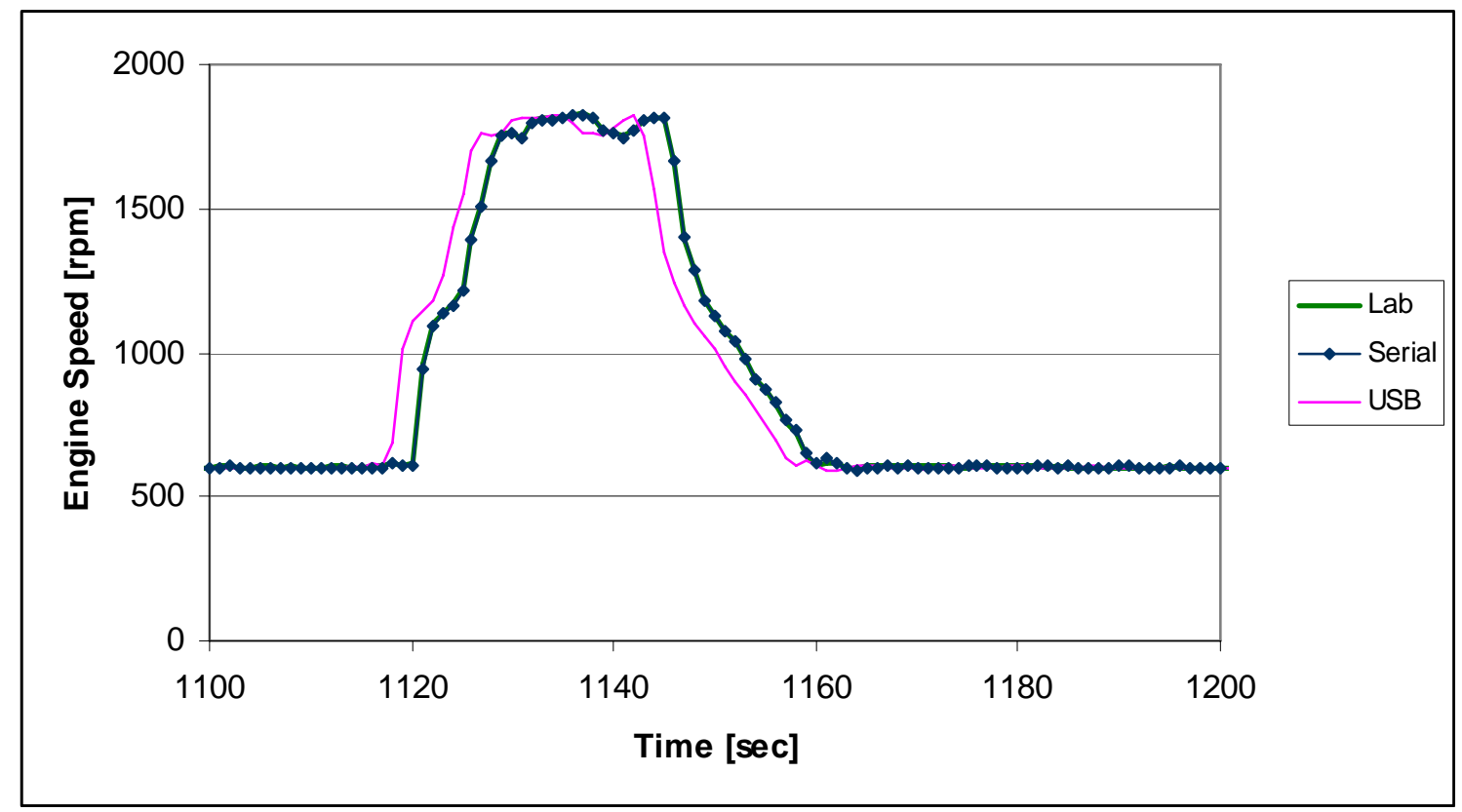

Figure 26 Engine speed Data Collected by the Lab, Serial DPA and USB DPA on 1708 link

Figure 27 shows an example of engine speed data collected during the Volvo engine tests with the DPA adapters connected to the J1939 link. Again, all three data sets were aligned at the beginning of the test and show some discrepancy at the end. In this case the USB DPA data show an error of roughly 15 seconds. This figure also shows an error due to insufficient frequency of data collection. This error occurs regardless of the type of DPA used. It is visible in the erratic idle section before the major power spike around 1120 sec. The DLM software was collecting all broadcasted ECU data and the frequency of engine speed data collection was insufficient. After parsing the data, discrepancies in 
high rate transients events become apparent as can be seen in Figure 27. However, this error is not the same issue this study is concerned about, and is easily remedied by filtering for desired data.

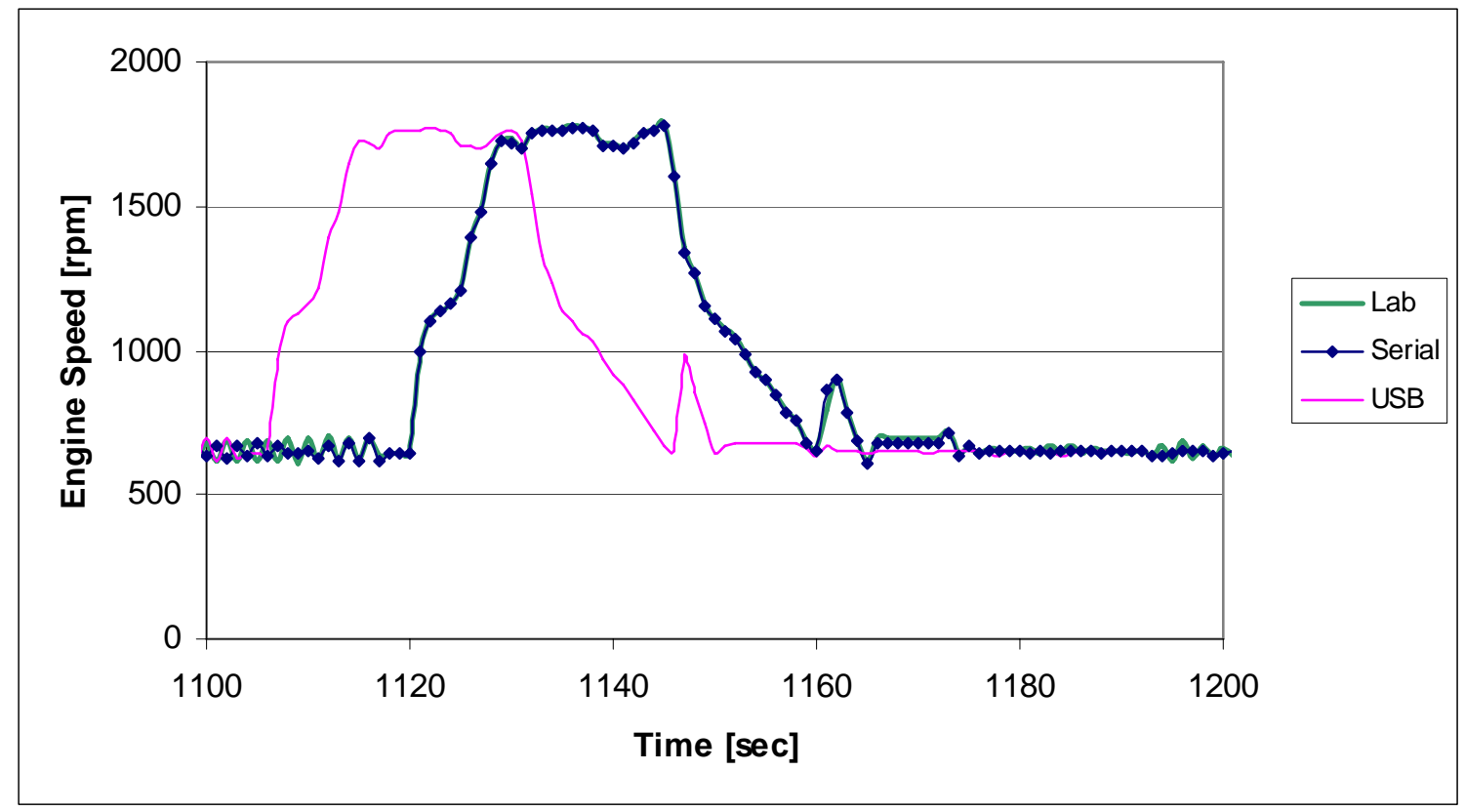

Figure 27 Engine speed Data Collected by the Lab, Serial DPA and USB DPA on 1939 link

The observed pattern of timestamp related error occurrence singles out the usage of USB DPA device as the only cause of it. It was not determined what exactly causes the error. It might be attributed to the DLM software itself or the USB device driver, or possibly the problem lies within the hardware components of the DPA4 itself. A detailed review of the data transfer processes and the software algorithms need to be conducted to pinpoint the core of the problem. However, work of this extent is beyond the scope of this study. The manufacturer, Dearborn Group, was notified of the problem, and the company expressed an interest in correcting it. 


\subsection{Error Correction Evaluation}

Visual inspection of the data revealed that the absolute time stamp error increases consistently during the test span without major sudden spurts. Hence, a strategy of correcting this error by linear stretch of the time stamp of the ECU data was adopted. This method of stretching was intended for correcting MEMS data that were obtained prior to the discovery of the problem and containing the error. The correction was achieved by modifying the raw DLM data file by procedure described in Section 4.3. To evaluate the accuracy of this correction method, several MEMS tests were conducted while collecting DLM data using both USB and serial DPA adapter. There were seven vehicles used for this study with 55 test runs collected between them. Six of these vehicles were equipped with engines produced by Manufacturer A and one was equipped with an engine form Manufacturer B. The MEMS test emission data were, as customary, reduced with the USB DPA-DLM ECU data. The USB DPA-DLM ECU data were then corrected using the adopted procedure and the emission data were reduced again with the corrected ECU data. Additionally, the emission data were reduced with the serial DPADLM ECU data. These serial data were used as a standard for comparison, since it was established that these are correct in terms of time. Appendix E includes tables of all the tests along with the correction factors needed for the USB DLM ECU data. The tables are sorted by the test vehicles and test weights of the vehicles.

The first comparison that was done with these data was to check how the power obtained from the corrected USB data compares with the power obtained from the serial DLM data. For each test run there was a regression analysis performed using these two sets of ECU-obtained power data and a linear trend line was placed on this regression analysis using the Microsoft Excel software. An example of regression analysis using uncorrected erroneous USB data can be seen in Figure 28. It is obvious, from this figure, 
that the uncorrected USB data have a large disagreement with the serial data. An example of a plot of regression analysis of the corrected USB data is in Figure 29, which also shows the linear trend line with its equation and the $\mathrm{R}^{2}$ value. This trend line indicates significantly closer match between the two data sets then in Figure 28.

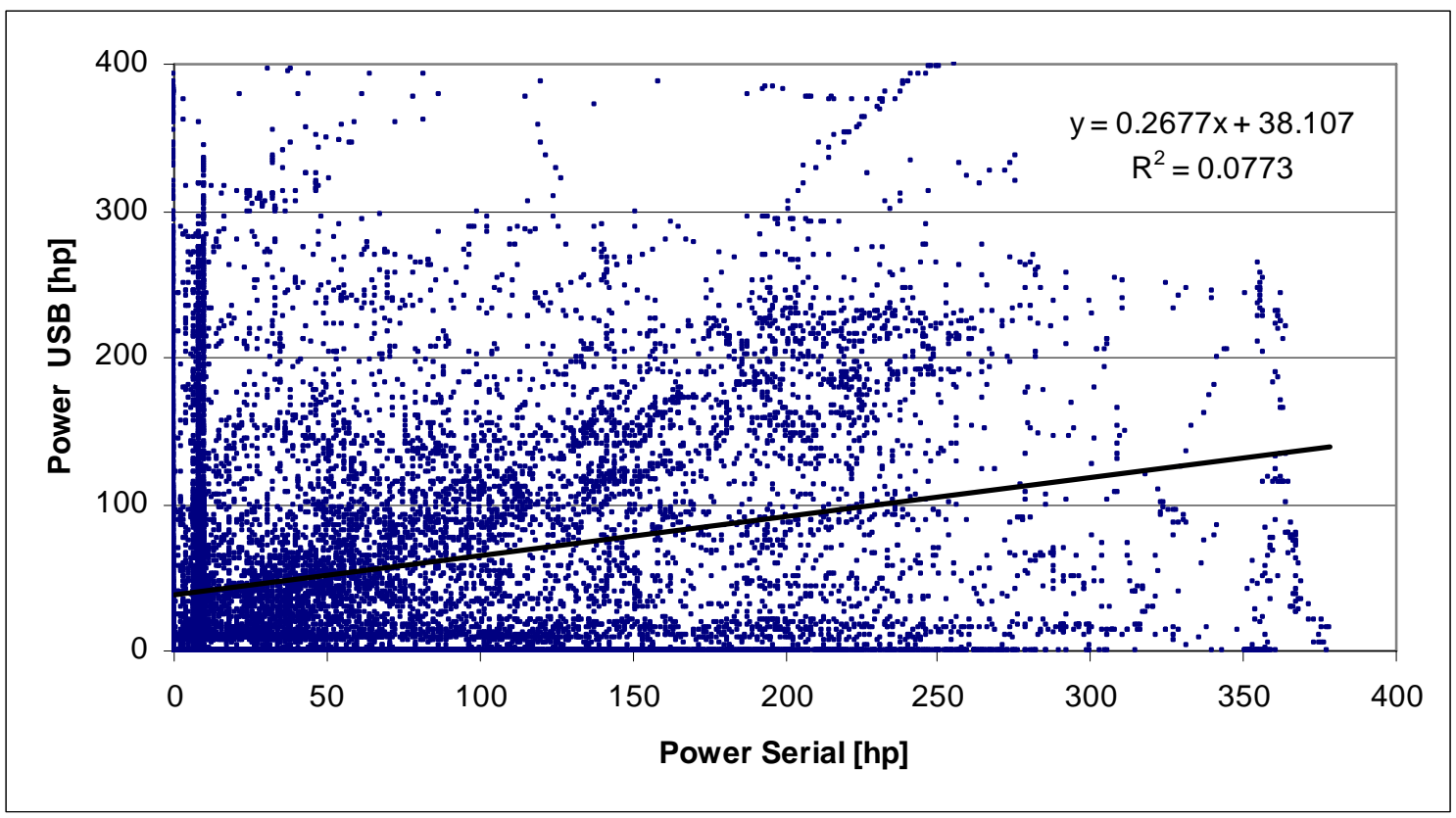

Figure 28 Regression Analysis of Engine Power with Erroneous USB Data and the Serial Data

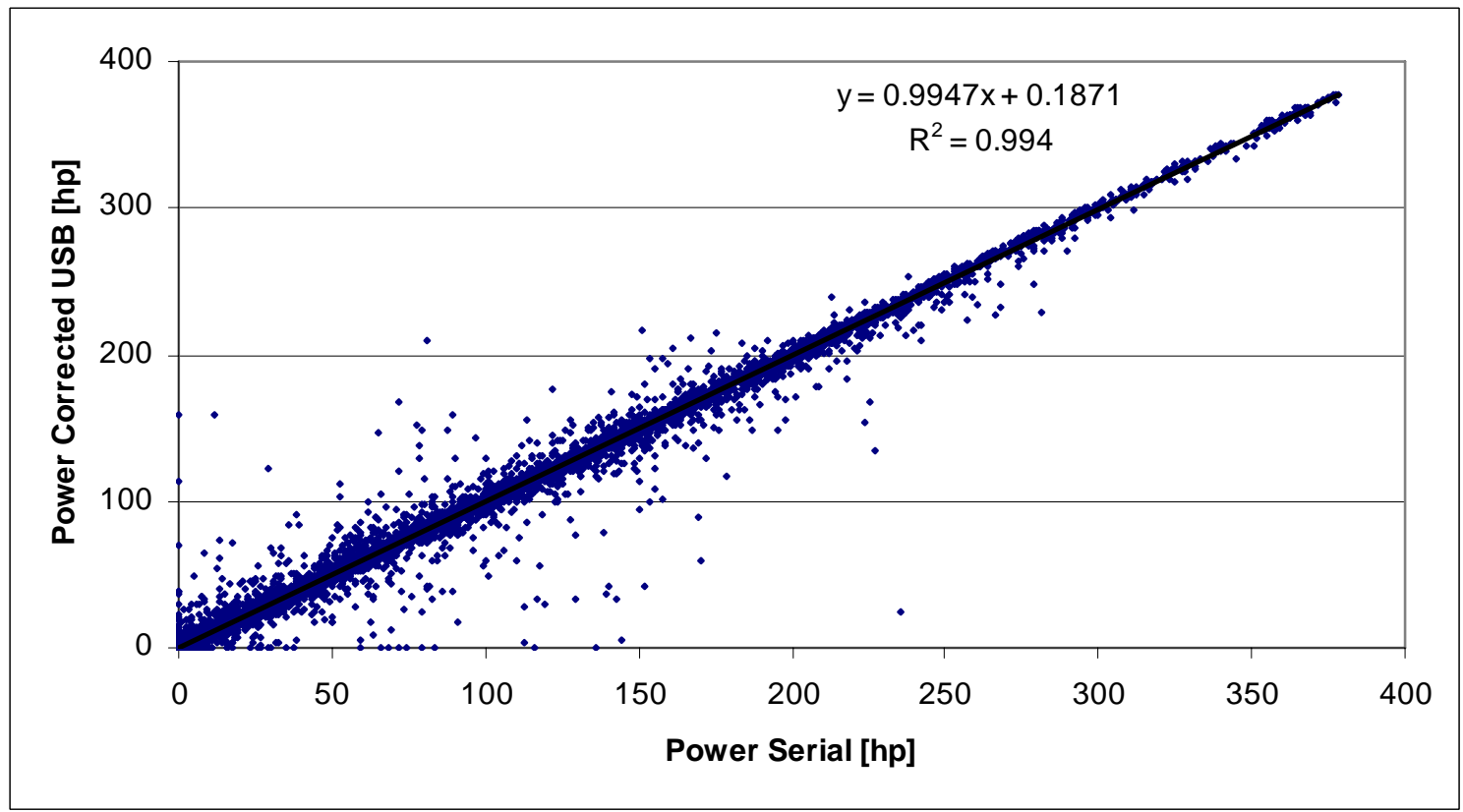

Figure 29 Regression Analysis of Corrected USB and Serial Engine Power Data 
Ideally, all the points of the regression would fall on a line generated by an equation $\mathrm{y}=$ $1 \mathrm{x}+0$ (in this equation $\mathrm{y}$ represents the corrected USB data and $\mathrm{x}$ represents the serial data) in which case the $\mathrm{R}^{2}$ would be 1 . The averaged equation of all the regression analysis from all the tests is $y=0.997 x+0.432$. Due to the fact that the DLM equipment does not collect every single data point broadcasted by the ECU there would be differences between two data sets even if they were collected by two identical adapters working correctly without any associated timing error. The data is simply collected as fast as it can be saved. The most obvious differences will be noticeable during highly transient operation where one adapter might miss a data point representing a sharp peak within the data stream. With this in mind, the power data sets were filtered for data with a rate of change smaller than $150 \mathrm{hp} / \mathrm{s}$ to discard the highly transient peaks to see if the regression analyses produce better matches. It should be noted that the data were filtered just for the sake of demonstrating that most of the differences occur in highly transient operation. These data are not of interest for in-use emissions testing since the emissions are only evaluated for at least 30 second continuous operation in NTE zones, which usually excludes highly transient processes such as transmission shifts or sudden traffic maneuvers of the vehicle. The resulting linear regression of filtered data produced better results as can be seen in Figure 30, which shows linear regression of filtered data from the same test as Figure 29. 


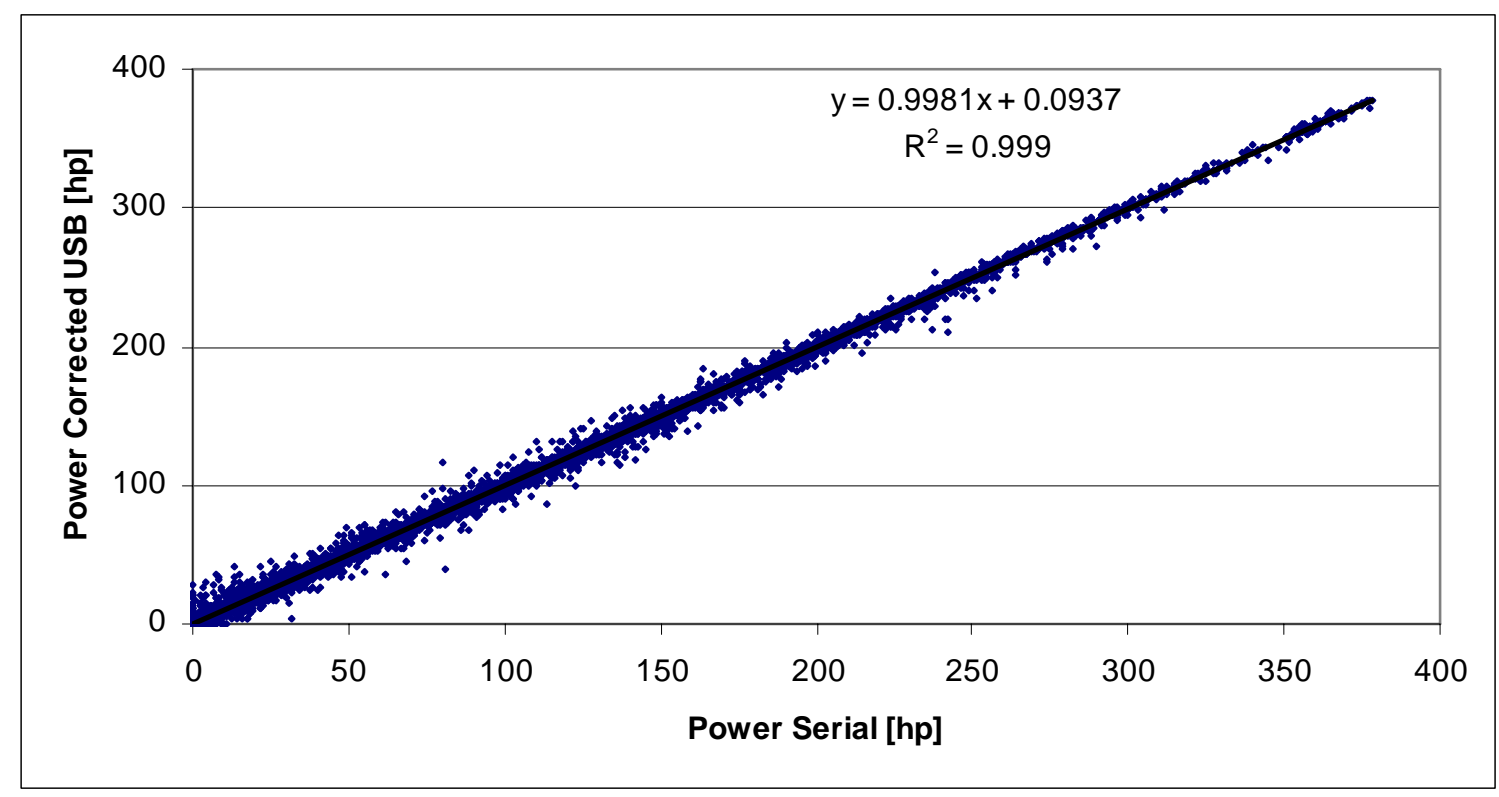

Figure 30 Regression Analysis of Filtered Corrected Engine Power Data

Table 7 is a summary of all regression analyses; it shows averaged coefficients (slope and intercept) of the linear regression trend line equations from all the collected tests and the averaged $\mathrm{R}^{2}$ values. It also shows the standard deviations from these averaged values as well as maximum and minimum for each parameter. A table, for all tests, including all coefficients is included in Appendix B.

Table 7 Summary of Regression Analyses Trend Line Equations of Serial and Corrected USB power

\begin{tabular}{|c|r|r|r|r|r|r|}
\hline & \multicolumn{3}{|c|}{ Unfiltered } & \multicolumn{3}{c|}{ Filtered } \\
\hline Coefficient & \multicolumn{1}{|c|}{ Slope } & Intercept & \multicolumn{1}{c|}{$\mathrm{R}^{2}$} & \multicolumn{1}{c|}{ Slope } & Intercept & $\mathrm{R}^{2}$ \\
\hline Average & 0.997 & 0.434 & 0.994 & 1.00 & 0.0327 & 0.999 \\
\hline Std Deviation & 0.00239 & 0.429 & 0.00496 & 0.00102 & 0.119 & 0.000725 \\
\hline Max & 1.00 & 1.76 & 1.00 & 1.00 & 0.458 & 1.00 \\
\hline Min & 0.990 & -0.0375 & 0.979 & 0.997 & -0.345 & 0.996 \\
\hline
\end{tabular}

The next evaluation of the corrected USB DLM data was done in terms of comparing actual brake specific emissions data. The MEMS measures the exhaust emissions of $\mathrm{CO}_{2}$ using the Horiba BE-140 analyzer, and as the primary interest, emission of NOx, which is measured by two different analyzers. These two are Horiba Mexa 720 zirconium oxide analyzer and Sensors Inc. electrochemical cell, which is only 
a quality control or back up device. One of the outputs from MEMS reduction software is the summary of the NTE emissions in terms of average, maximum, and minimum values of brake specific emissions of the pollutants. These values are reported for continuous NTE events and for separate 30 second NTE windows. MEMS records data at frequency of $5 \mathrm{~Hz}$ so, for example, a 31 second long continuous NTE event can contain 6 separate 30 second long NTE windows, each starting with a new data point as these are .2 second apart. The NTE emissions can then be evaluated for each 30 second window separate or for one continuous event. The continuous event evaluation averages out any temporarily extremes of emission production that would be otherwise apparent from evaluation of each 30 second window. Whether an engine passes the emission test is decided by the maximum NTE MEXA NOx value obtained from the individual 30 second windows. For each test, the emissions were reduced three times using the USB DLM data, the corrected USB DLM data and the serial DLM ECU data. Emissions data reduced with the USB DLM data and the corrected USB DLM data were compared to emissions data reduced with the serial DLM data, which were assumed correct for the purpose of comparison. Due to the fact that the timing error of the USB DLM data caused emissions data to be paired up with random power data, results of these comparisons are somewhat random, as well. There is no apparent significant trend to the amount of error projected into brake specific emission values. Obviously there is some dependence of the resulting emission error on the level of inaccuracy of the ECU data time stamps and the length of the test. Given the same rate of time "shrinkage" of the ECU data, a longer test will result in larger misalignment of emissions and power data. Likewise, given the same length of test, higher rate of time "shrinkage” will also result in larger misalignment of emissions and power data. Figure 31 shows the dependence (or lack of it) of error (\%) in maximum value of brake specific NTE MEXA NOx emission on the length of the test or the amount 
of "shrinkage"of the USB DLM data. In this chart each bubble represents a value of error from one test. The size of the bubble indicates the amount of the error, the largest bubble in this chart denotes $47 \%$. The error is based on comparison to emission data reduced using serial DLM ECU data. The vertical axis indicates the length of a particular test in seconds. The horizontal axis indicates the amount of time "shrinkage" in the DLM ECU data of a particular test in terms of correction factor needed to stretch the data to appropriate length by multiplying the time stamps. While Figure 31 shows the errors in maximum MEXA NOx emissions on a 30 second NTE window basis, Figure 32 shows the same relationship, but on a continuous NTE window basis. Notice that the size of the bubbles indicates the amount of error within each chart but does not correlate between the two charts. While the largest bubble in Figure 31 represents 47\% error, in Figure 32 the largest bubble represent only $11 \%$ error.

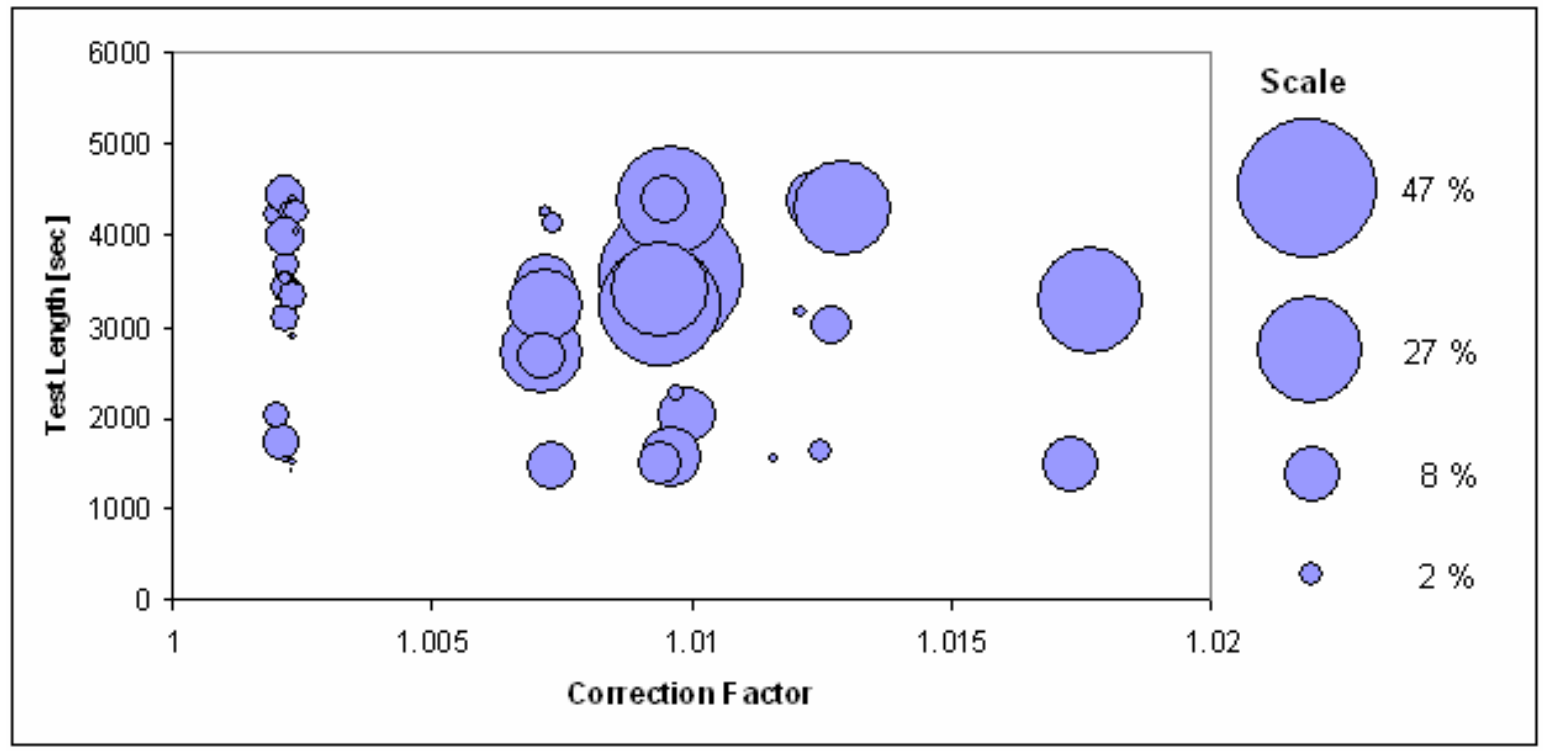

Figure 3130 sec NTE Maximum MEXA NOx Error - Dependence on Test Length and Amount of "Shrinkage" in USB DLM Data 


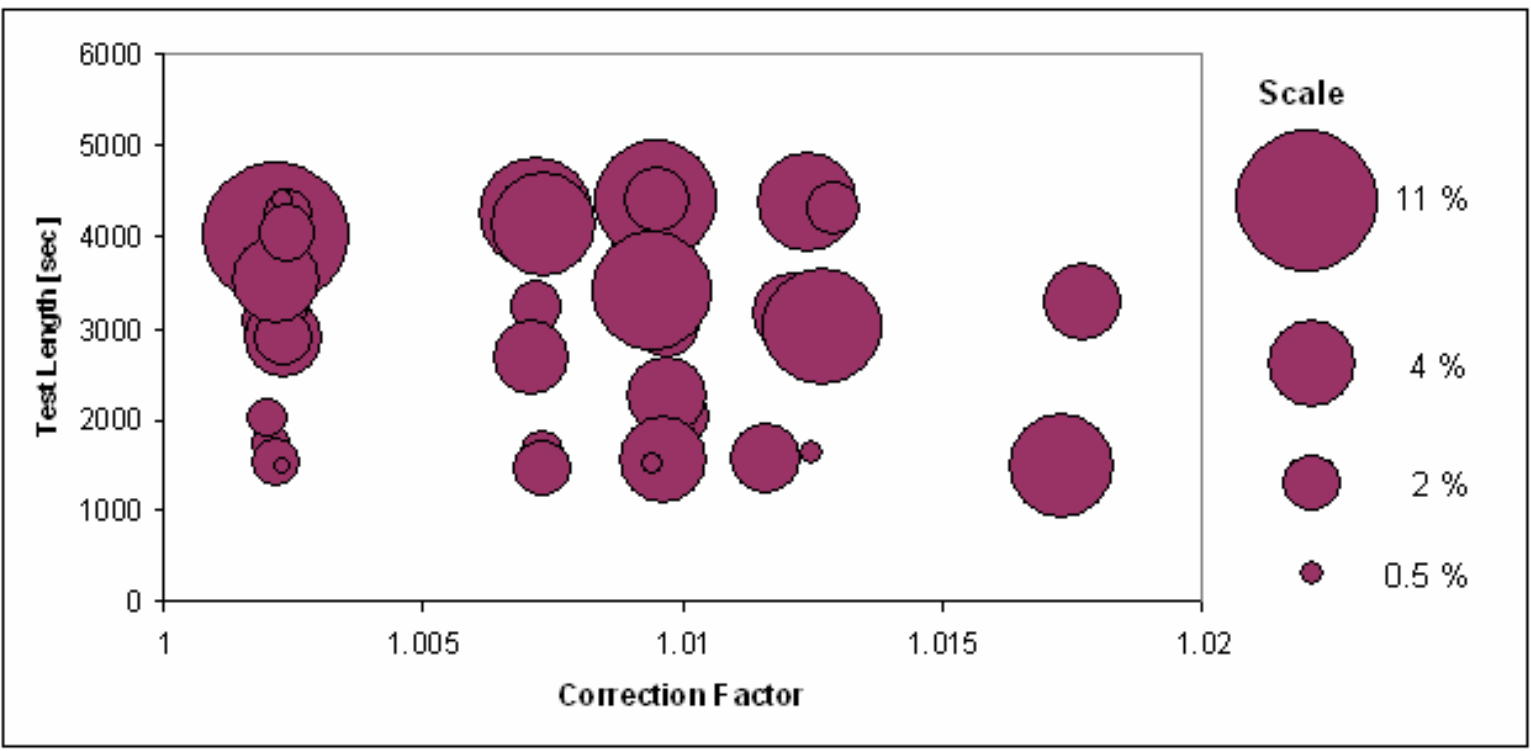

Figure 32 Continuous NTE Maximum MEXA NOx Error - Dependence on Test Length and Amount of "Shrinkage" in USB DLM Data

Correcting the time stamp error in the USB DLM data results in significant improvement in accuracy of the brake specific emission values. Figure 33 shows the absolute percent errors in maximum MEXA NOx emissions for each test. The error is calculated with the emissions data reduced with the serial DLM data as a correct standard. The blue bars represent percent error in emission data reduced with the USB DLM data, while the purple bars represent the percent error in emission data reduced with the corrected USB DLM data. Figure 34 shows the same data as Figure 33 with focus on the bottom two percent error range to see more detail as all the errors in emission values obtained with the corrected USB data are rather small. 


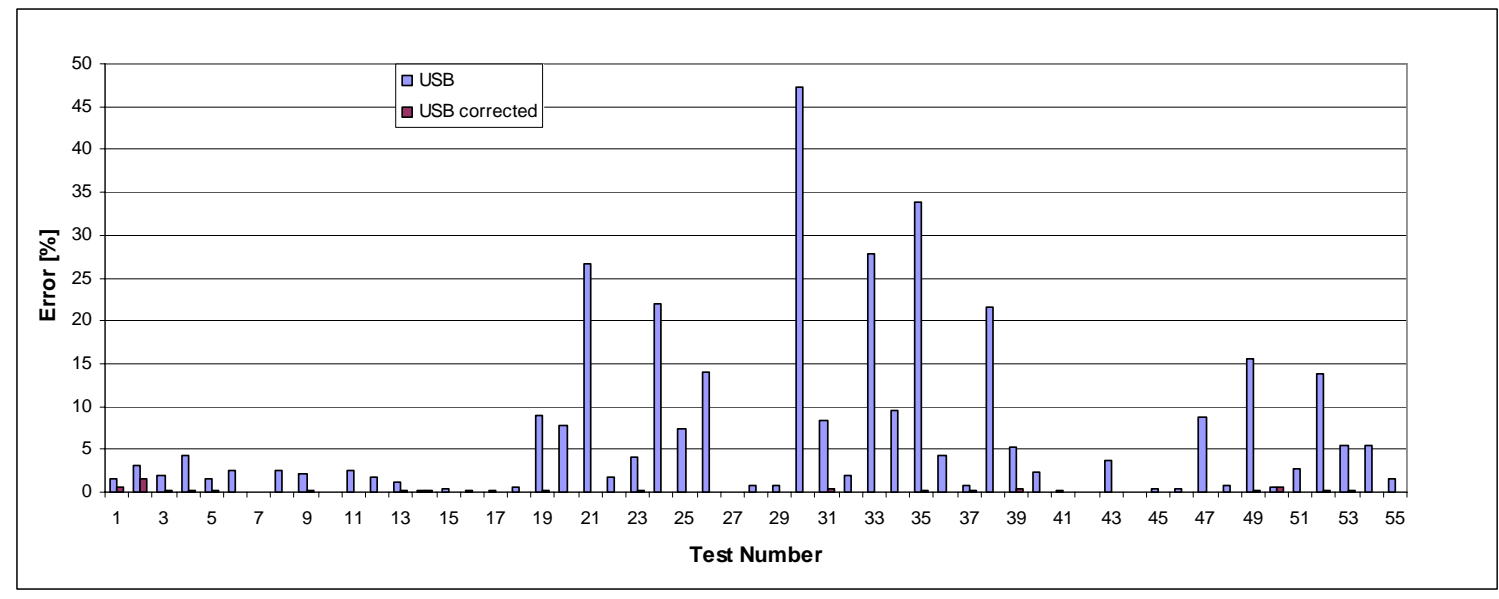

Figure 33 Error in Maximum MEXA NOx Brake Specific NTE Emissions

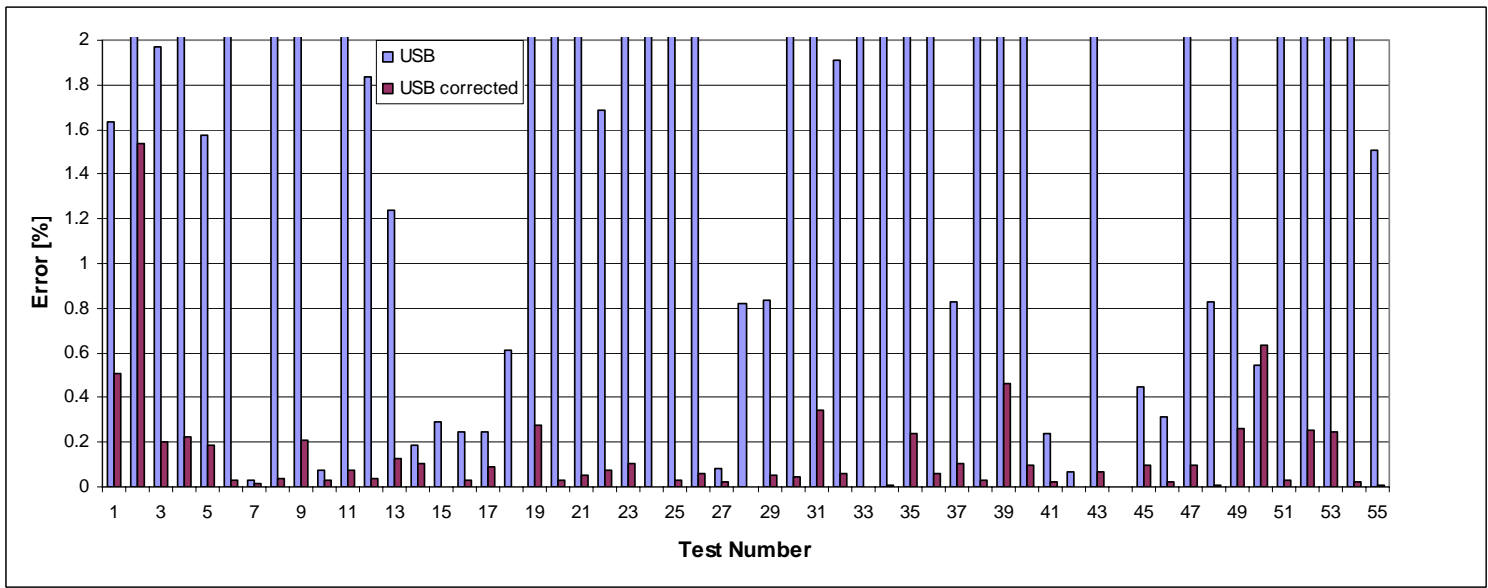

Figure 34 Detail of Error in Maximum MEXA NOx Brake Specific NTE Emissions

The average absolute error in brake specific maximum 30 second NTE NOx emission in data reduced with USB DLM data was $6.24 \%$ with maximum value of 47.34 $\%$. The same emissions data produced an average error of $0.134 \%$ with maximum peaking at $1.54 \%$ when reduced with the corrected USB data. A complete table of these error data is included in Appendix C. Table 8 identifies the engines of tested vehicles with corresponding test numbers in the previous two figures. 
Table 8 Vehicles and Engines with Corresponding Test Numbers

\begin{tabular}{|c|c|c|}
\hline Test No. & $\begin{array}{c}\text { Engine } \\
\text { Manufacturer }\end{array}$ & Engine Year \\
\hline $1 \sim 9$ & A & 2001 \\
\hline $10 \sim 16$ & A & 2001 \\
\hline $17 \sim 19$ & A & 2001 \\
\hline $20 \sim 24$ & A & 2001 \\
\hline $25 \sim 39$ & B & 2002 \\
\hline $40 \sim 46$ & A & 2001 \\
\hline $47 \sim 55$ & A & 2001 \\
\hline
\end{tabular}

Table 9 shows the test results for all the tests conducted on vehicles equipped with 2001 Manufacturer A engines. This table shows the maximum 30 second NTE emissions of NOx calculated with the Serial, the USB and the Corrected USB DLM data and indicates whether the engine satisfied the in-use emission limits with any of the data sets used. The NTE threshold of passing the emission test for all vehicles tested for this study is $7.5 \mathrm{~g} / \mathrm{bhp}-\mathrm{hr}$ of NOx. The NTE threshold is calculated as 1.75 times the FTP limit [4] plus 0.5 g/bhp-hr measurement allowance [17]. 
Table 9 Summary of NOx Max Test Results for Vehicles with 2001 Manufacturer A Engines

\begin{tabular}{|c|c|c|c|c|c|c|c|c|}
\hline No. & $\begin{array}{c}\text { Serial } \\
\text { Nox } \\
\text { Max } \\
\text { g/bhp- } \\
\text { hr }\end{array}$ & $\begin{array}{c}\text { USB } \\
\text { Nox } \\
\text { Max } \\
\text { g/bhp- } \\
\text { hr }\end{array}$ & $\begin{array}{c}\text { USB } \\
\text { Corr. } \\
\text { Nox } \\
\text { Max } \\
\text { g/bhp- } \\
\text { hr }\end{array}$ & $\begin{array}{l}\text { Limit } \\
\text { FTP } \\
\text { g/bhp- } \\
\text { hr }\end{array}$ & $\begin{array}{l}\text { Limit } \\
\text { In-Use } \\
\text { g/bhp- } \\
\text { hr }\end{array}$ & $\begin{array}{l}\text { Test } \\
\text { Pass } \\
\text { Serial }\end{array}$ & $\begin{array}{l}\text { Test } \\
\text { Pass } \\
\text { USB }\end{array}$ & $\begin{array}{l}\text { Test } \\
\text { Pass } \\
\text { USB } \\
\text { Corr }\end{array}$ \\
\hline 1 & 7.02 & 7.14 & 7.06 & 4 & 7.5 & yes & yes & yes \\
\hline 2 & 4.65 & 4.50 & 4.57 & 4 & 7.5 & yes & yes & yes \\
\hline 3 & 6.30 & 6.43 & 6.29 & 4 & 7.5 & yes & yes & yes \\
\hline 4 & 6.39 & 6.66 & 6.41 & 4 & 7.5 & yes & yes & yes \\
\hline 5 & 7.35 & 7.47 & 7.36 & 4 & 7.5 & yes & yes & yes \\
\hline 6 & 6.66 & 6.83 & 6.66 & 4 & 7.5 & yes & yes & yes \\
\hline 7 & 6.43 & 6.43 & 6.43 & 4 & 7.5 & yes & yes & yes \\
\hline 8 & 6.29 & 6.14 & 6.29 & 4 & 7.5 & yes & yes & yes \\
\hline 9 & 6.61 & 6.75 & 6.60 & 4 & 7.5 & yes & yes & yes \\
\hline 10 & 3.57 & 3.57 & 3.57 & 4 & 7.5 & yes & yes & yes \\
\hline 11 & 6.04 & 5.89 & 6.04 & 4 & 7.5 & yes & yes & yes \\
\hline 12 & 5.75 & 5.64 & 5.75 & 4 & 7.5 & yes & yes & yes \\
\hline 13 & 5.77 & 5.70 & 5.78 & 4 & 7.5 & yes & yes & yes \\
\hline 14 & 3.72 & 3.73 & 3.73 & 4 & 7.5 & yes & yes & yes \\
\hline 15 & 5.76 & 5.77 & 5.76 & 4 & 7.5 & yes & yes & yes \\
\hline 16 & 5.85 & 5.86 & 5.85 & 4 & 7.5 & yes & yes & yes \\
\hline 40 & 6.46 & 6.31 & 6.47 & 4 & 7.5 & yes & yes & yes \\
\hline 41 & 3.50 & 3.50 & 3.49 & 4 & 7.5 & yes & yes & yes \\
\hline 42 & 5.74 & 5.73 & 5.74 & 4 & 7.5 & yes & yes & yes \\
\hline 43 & 6.27 & 6.03 & 6.26 & 4 & 7.5 & yes & yes & yes \\
\hline 44 & 5.49 & 5.49 & 5.49 & 4 & 7.5 & yes & yes & yes \\
\hline 45 & 5.69 & 5.72 & 5.69 & 4 & 7.5 & yes & yes & yes \\
\hline 46 & 5.98 & 5.96 & 5.98 & 4 & 7.5 & yes & yes & yes \\
\hline 47 & 8.77 & 8.01 & 8.76 & 4 & 7.5 & no & no & no \\
\hline 48 & 4.55 & 4.51 & 4.55 & 4 & 7.5 & yes & yes & yes \\
\hline 49 & 6.13 & 7.08 & 6.14 & 4 & 7.5 & yes & yes & yes \\
\hline 50 & 7.83 & 7.79 & 7.88 & 4 & 7.5 & no & no & no \\
\hline 51 & 5.96 & 6.11 & 5.96 & 4 & 7.5 & yes & yes & yes \\
\hline 52 & 6.53 & 7.43 & 6.54 & 4 & 7.5 & yes & yes & yes \\
\hline 53 & 4.64 & 4.39 & 4.63 & 4 & 7.5 & yes & yes & yes \\
\hline 54 & 5.95 & 6.27 & 5.95 & 4 & 7.5 & yes & yes & yes \\
\hline 55 & 5.86 & 5.95 & 5.86 & 4 & 7.5 & yes & yes & yes \\
\hline 17 & 2.35 & 2.36 & 2.36 & 4 & 7.5 & yes & yes & yes \\
\hline 18 & 3.01 & 3.03 & 3.01 & 4 & 7.5 & yes & yes & yes \\
\hline 19 & 2.96 & 3.23 & 2.95 & 4 & 7.5 & yes & yes & yes \\
\hline 20 & 3.19 & 3.43 & 3.19 & 4 & 7.5 & yes & yes & yes \\
\hline 21 & 3.09 & 3.92 & 3.09 & 4 & 7.5 & yes & yes & yes \\
\hline 22 & 2.42 & 2.38 & 2.41 & 4 & 7.5 & yes & yes & yes \\
\hline 23 & 3.12 & 3.24 & 3.12 & 4 & 7.5 & yes & yes & yes \\
\hline 24 & 3.14 & 3.84 & 3.14 & 4 & 7.5 & yes & yes & yes \\
\hline
\end{tabular}


Table 10 shows the same information as Table 9, but for tests of one vehicle equipped with 2002 Manufacturer B engine.

Table 10 Summary of NOx Max Test Results for a Vehicle with the 2002 Manufacturer B Engine

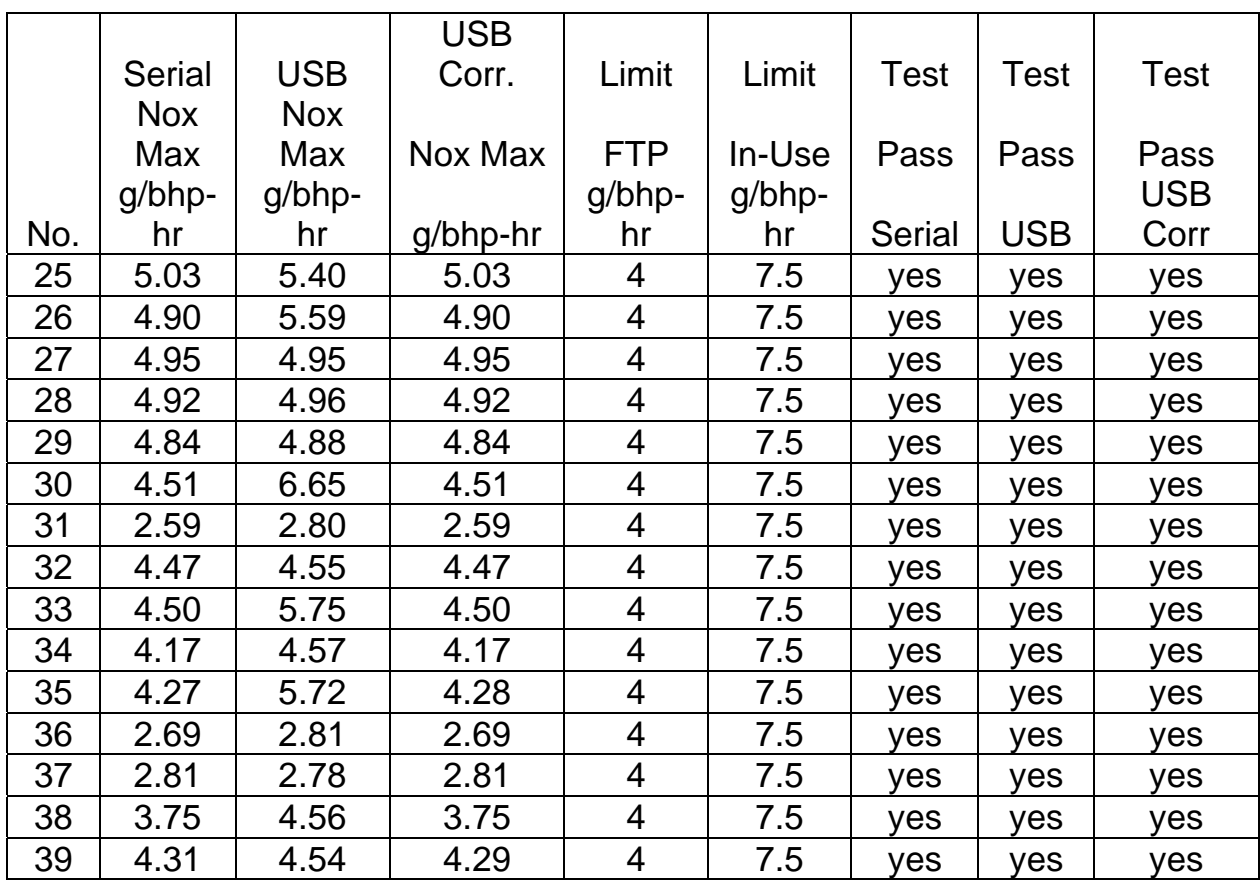

Notably, none of the vehicles tested in this study experienced a change in the status of the test result. One vehicle failed two tests, regardless of which set of DLM ECU data was used for brake specific emissions reduction. Due to the generous NTE NOx emissions threshold (1.75 x FTP value +0.5 g/bhp-hr) set for the pre Oct. 2002 engines, most vehicles passed most of the tests without even approaching the limit. 


\section{Conclusions and Recommendations}

The objectives of this study were to examine the timing error found in ECU data collected with DPA hardware and DLM software, to determine under what conditions the error occurs, and to find solutions for avoiding and correcting this error.

\subsection{Error Occurrence}

A series of tests was conducted to determine under what circumstances exactly does the discussed error occur. Two engines were used for this study from different manufacturers and of distant year of manufacture. One was a pre Consent Decree engine broadcasting J1587 protocol only and the other was a post Consent Decree engine broadcasting both J1587 and J1939 protocols. Numerous DPA units were used for the testing. Two types, the DPA III+/T with serial communication and the DPA4 with USB communications, were used. In addition, two different data acquisition computers equipped with the DLM data collection software were used and the testing was conducted with various hardware configurations. The outcome of this study was the conclusion that the error is caused by incorrect time stamp assignment associated only with usage of the USB communicating DPA4. It was not determined, from data collected in this s study, what exactly causes the error within the operation of the DPA4 and/or DLM. The error could be attributed to the hardware (DPA4) or to the software side of the equipment. Since this equipment was not designed or built at WVU, the problem correction to the extent of redesign and modification of the equipment was not attempted. However, the manufacturer, Dearborn Group, was contacted and informed about their product performance problem. The manufacturer expressed lack of knowledge of this problem and indicated that it will be investigated.

The results of this study suggest that usage of DPA4 is not desirable, and therefore it is recommended that DPAIII+/T be used instead, regardless of the inconveniences of 
additional data acquisition computer needed during MEMS testing. This practice should be followed until the manufacturer solves the problem.

\subsection{Error Correction for MEMS Data Reduction Application}

In this part of the study, a method was developed to correct MEMS test data that are affected with the error caused by using the DPA4with USB communication. Data necessary for achieving this goal were collected during several MEMS tests. In addition to the MEMS customary USB DPA/DLM ECU data acquisition system, an additional serial DPA/DLM ECU data acquisition system was employed. Data collected with this additional equipment was used for evaluation of the correction method developed in this study. The correction was accomplished by stretching the time axis of the ECU data through modification to the raw DLM file; the magnitude of stretch necessary was determined by comparing the ECU obtained power data to the independently acquired mass rate $\mathrm{CO}_{2}$ data. Resulting brake specific 30 second NTE maximum NOx emissions from the on-road MEMS tests were examined, since these values are the determining factor engine in-use compliance. With the correction, the average error of these values dropped from $6.24 \%$ to $0.134 \%$ and the maximum error was reduced from $47.4 \%$ down to $1.54 \%$.

As the post-correction error values were very low and well under the $0.5 \mathrm{~g} / \mathrm{bhp}-\mathrm{hr}$ allowance limit for in-use testing, it was concluded that this correction method was acceptable. 


\section{References}

1. Emission Standards: United States - Heavy Duty Truck Engines, Retrieved Jan 2007 from www.dieselnet.com, Ecopoint Inc.

2. Download: DPA Pinout Assignments, Retrieved Jan 2007 from www.dgtech.com, Dearborn Group, Inc.

3. Moynahan N.A. , 2005, "Development of a Vehicle Road Load Model for ECU Broadcast Power Verification in On-Road Emission Testing,” M.S. Thesis, Department of Mechanical and Aerospace Engineering, West Virginia University, Morgantown, WV.

4. Thompson G.J., Clark N.N., Gautam M., Carder D.K. and Lyons D.W. , 2002, "Inference of Torque and Power from Heavy-Duty Diesel Engines for On-road Emissions Monitoring, “ SAE Technical Paper No. 2002-01-0614Cases: Information Resources, Compliance \& Enforcement: Consent Decrees, Retrieved Feb 2007 from www.epa.gov, U.S. Environmental Protection Agency.

5. Deutsch 6-Pin to DB15 Male Cable, Retrieved Feb 2007 from http://www.autotap.com/files/D6D15M-1303ds.pdf, B\&B Electronics.

6. Deutsch 9-Pin to DB15 Male Cable, Retrieved Feb 2007 from http://www.autotap.com/files/D9D15M-1303ds.pdf, B\&B Electronics.

7. Roadranger- Eaton Fuller Automated Transmissions, Retrieved Feb 2007 from http://www.roadranger.com/ecm/groups/public/@pub/@roadranger/documents/conte nt/rr_trig-2500.pdf, Roadranger.

8. Gautam M., Clark N.N., Thompson G.J., Carder D.K. and Lyons D.W., 2000, "Evaluation of Mobile Monitoring Technologies for Heavy-Duty Diesel-Powered Vehicle Emissions,” Report Submitted to Settling-Heavy Duty Diesel Engine Manufacturers, Department of Mechanical and Aerospace Engineering, West Virginia University, Morgantown, WV.

9. "Serial Data Communications between Microcomputer Systems in Heavy-Duty Vehicle Applications,” 1993, SAE Standard, SAE J1708.

10. SAE Standard 1996, “Joint SAE/TMC Electronic Data Interchange between Microcomputer Systems in Heavy-Duty Vehicle Applications,” SAE J1587.

11. SAE Standard 1994, "Physical Layer-250 Kbits/s, Shielded Twisted Pair," SAE J1939.

12. SAE Standard 1996, "Vehicle Application Layer," SAE J1939/71

13. “DDEC II Application and Instalation Manual,” 1991, Detroit Diesel Corp.

14. Heck E.T., Kitzerow J. and Carvella T., 1991, "Large Scale Application of J-1939 CAN,” SAE Technical Paper No.1991-01-2840.

15. “National Air Pollutant Emission Trends, 1900-1998,” Retrieved Apr 2007 from http://www.epa.gov/ttn/chief/trends/trends98/trends98.pdf, EPA.

16. "Heavy Duty Engine and Vehicle Standards and Highway Diesel Fuel Sulfur Control Requirements,” Retrieved Apr 2007 from http://www.epa.gov/otaq/regs/hd2007/frm/f00057.pdf, EPA.

17. Shimpi S.A., 2007, "US Engine Manufacturer Views on In-Use Testing," Proceedings of the JRC Transport and Emissions Conference, Retrieved May 2007 from http://transportenv07.jrc.it/pdf/C/C_1_19_Shimpi_S_US\%20Manufacturer\%20Views \%20on\%20In-Use\%20Emissions.pdf, JRC. 


\section{Appendix A}

\section{Matlab Program for the DLM File Time Stamp Error Correction}

(Program written by Rafaello Ardanese, Dec. 2006)

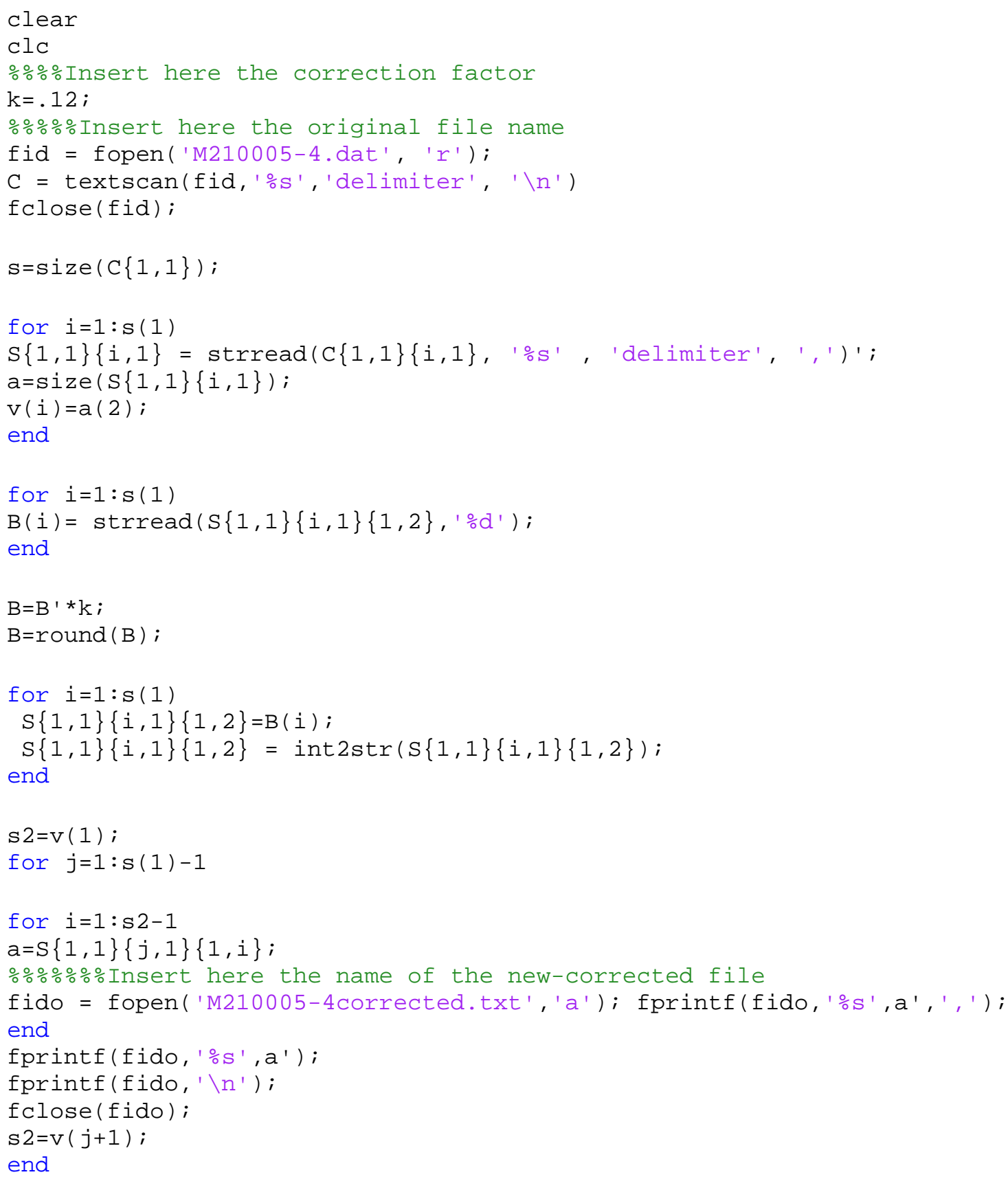




\section{Appendix B}

\section{Compete Table of Linear Regression Trend Line Equation Coefficients of Serial and}

Corrected USB Power Data for Each Test

\begin{tabular}{|c|c|c|c|c|c|c|}
\hline \multirow[b]{2}{*}{ Test number } & \multicolumn{3}{|l|}{ Unfiltered } & \multicolumn{3}{|l|}{ Filtered } \\
\hline & Slope & Intercept & $\mathrm{R}^{2}$ & Slope & Intercept & $\mathrm{R}^{2}$ \\
\hline 1 & 9.97E-01 & 4.67E-01 & 9.95E-01 & $1.00 \mathrm{E}+00$ & 1.13E-01 & $1.00 \mathrm{E}+00$ \\
\hline 2 & 9.99E-01 & 3.76E-01 & 9.95E-01 & $1.00 \mathrm{E}+00$ & $-3.56 E-02$ & $1.00 \mathrm{E}+00$ \\
\hline 3 & 9.98E-01 & 5.25E-01 & 9.92E-01 & $1.00 \mathrm{E}+00$ & 2.45E-02 & $9.99 \mathrm{E}-01$ \\
\hline 4 & 9.99E-01 & 1.12E-01 & 9.98E-01 & $1.00 \mathrm{E}+00$ & $-2.39 E-02$ & $1.00 \mathrm{E}+00$ \\
\hline 5 & 9.97E-01 & 2.05E-01 & 9.92E-01 & 9.99E-01 & 2.59E-02 & 9.99E-01 \\
\hline 6 & 9.96E-01 & $6.25 \mathrm{E}-01$ & $9.92 \mathrm{E}-01$ & $1.00 \mathrm{E}+00$ & 4.57E-02 & $1.00 \mathrm{E}+00$ \\
\hline 7 & 9.94E-01 & $1.04 \mathrm{E}+00$ & 9.82E-01 & $1.00 \mathrm{E}+00$ & $-4.70 \mathrm{E}-02$ & $9.99 \mathrm{E}-01$ \\
\hline 8 & 9.97E-01 & 5.67E-01 & 9.89E-01 & $1.00 \mathrm{E}+00$ & -1.02E-01 & 9.99E-01 \\
\hline 9 & 9.98E-01 & 3.62E-01 & 9.95E-01 & $1.00 \mathrm{E}+00$ & 6.95E-02 & $1.00 \mathrm{E}+00$ \\
\hline 10 & 9.97E-01 & 1.22E-01 & 9.97E-01 & 9.99E-01 & $-9.91 E-02$ & $1.00 \mathrm{E}+00$ \\
\hline 11 & 9.95E-01 & 7.92E-01 & 9.88E-01 & $1.00 \mathrm{E}+00$ & $-1.96 \mathrm{E}-01$ & 9.99E-01 \\
\hline 12 & 9.96E-01 & 7.37E-01 & 9.92E-01 & $1.00 \mathrm{E}+00$ & 3.15E-01 & $1.00 \mathrm{E}+00$ \\
\hline 13 & 9.94E-01 & $1.03 E+00$ & 9.88E-01 & 9.99E-01 & $-3.45 E-01$ & 9.99E-01 \\
\hline 14 & 9.97E-01 & $9.15 \mathrm{E}-02$ & 9.98E-01 & $9.99 \mathrm{E}-01$ & $3.77 \mathrm{E}-02$ & $1.00 \mathrm{E}+00$ \\
\hline 15 & 9.99E-01 & 2.39E-02 & 9.98E-01 & 9.99E-01 & $-3.56 \mathrm{E}-02$ & $1.00 \mathrm{E}+00$ \\
\hline 16 & 9.90E-01 & $1.64 \mathrm{E}+00$ & $9.79 \mathrm{E}-01$ & $1.00 \mathrm{E}+00$ & $4.58 \mathrm{E}-01$ & 9.98E-01 \\
\hline 40 & 9.98E-01 & 3.53E-01 & 9.95E-01 & $1.00 \mathrm{E}+00$ & 7.70E-03 & 9.99E-01 \\
\hline 41 & 9.98E-01 & 7.09E-01 & 9.91E-01 & $1.00 E+00$ & $-5.34 \mathrm{E}-02$ & 9.99E-01 \\
\hline 42 & 9.96E-01 & 8.14E-01 & 9.90E-01 & $1.00 E+00$ & 8.37E-02 & $9.98 \mathrm{E}-01$ \\
\hline 43 & 9.97E-01 & 5.30E-01 & 9.95E-01 & 9.99E-01 & $-1.23 E-01$ & $1.00 \mathrm{E}+00$ \\
\hline 44 & 9.96E-01 & 9.61E-02 & 9.96E-01 & 9.98E-01 & $-1.05 E-01$ & $1.00 \mathrm{E}+00$ \\
\hline 45 & 9.91E-01 & 6.81E-01 & 9.84E-01 & 9.99E-01 & 7.85E-02 & 9.99E-01 \\
\hline 46 & 9.96E-01 & 7.42E-01 & 9.94E-01 & $1.00 \mathrm{E}+00$ & 5.60E-02 & $1.00 \mathrm{E}+00$ \\
\hline 47 & 9.99E-01 & 1.24E-01 & 9.99E-01 & $1.00 \mathrm{E}+00$ & 1.18E-02 & $1.00 \mathrm{E}+00$ \\
\hline 48 & 9.98E-01 & 3.87E-02 & 9.98E-01 & 9.99E-01 & 3.06E-02 & $1.00 E+00$ \\
\hline 49 & 9.92E-01 & 4.45E-01 & 9.87E-01 & 9.97E-01 & 1.24E-01 & 9.98E-01 \\
\hline 50 & 9.98E-01 & 3.61E-01 & 9.96E-01 & $1.00 E+00$ & $-3.47 \mathrm{E}-02$ & $1.00 \mathrm{E}+00$ \\
\hline 51 & $1.00 \mathrm{E}+00$ & $-3.75 E-02$ & 9.99E-01 & $1.00 \mathrm{E}+00$ & $-2.16 E-02$ & $1.00 \mathrm{E}+00$ \\
\hline 52 & 9.93E-01 & $1.74 \mathrm{E}+00$ & 9.86E-01 & $1.00 \mathrm{E}+00$ & 2.30E-01 & 9.99E-01 \\
\hline 53 & 9.98E-01 & 3.35E-02 & 9.98E-01 & 9.99E-01 & 1.95E-02 & $1.00 \mathrm{E}+00$ \\
\hline 54 & 9.92E-01 & $1.20 \mathrm{E}+00$ & 9.82E-01 & $1.00 E+00$ & 5.44E-02 & 9.99E-01 \\
\hline 55 & 9.99E-01 & 1.76E-01 & 9.98E-01 & $1.00 \mathrm{E}+00$ & $-3.34 \mathrm{E}-02$ & $1.00 \mathrm{E}+00$ \\
\hline 17 & 9.94E-01 & 2.63E-01 & 9.91E-01 & 9.98E-01 & 1.54E-01 & 9.96E-01 \\
\hline 18 & 9.97E-01 & 1.06E-01 & 9.95E-01 & $1.00 \mathrm{E}+00$ & 8.44E-02 & 9.99E-01 \\
\hline 19 & 9.99E-01 & 1.06E-01 & 9.97E-01 & 9.99E-01 & $1.28 \mathrm{E}-01$ & 9.98E-01 \\
\hline 20 & $1.00 \mathrm{E}+00$ & -1.72E-02 & $1.00 \mathrm{E}+00$ & $1.00 \mathrm{E}+00$ & $-1.30 \mathrm{E}-03$ & $1.00 \mathrm{E}+00$ \\
\hline 21 & $1.00 \mathrm{E}+00$ & 5.64E-02 & 9.99E-01 & $1.00 E+00$ & $-1.09 E-02$ & 9.99E-01 \\
\hline 22 & 9.99E-01 & 1.74E-02 & 9.99E-01 & 9.99E-01 & 1.71E-02 & 9.99E-01 \\
\hline 23 & 9.97E-01 & 5.29E-02 & 9.96E-01 & 9.99E-01 & 8.55E-02 & 9.98E-01 \\
\hline 24 & 9.98E-01 & $1.72 \mathrm{E}-01$ & 9.97E-01 & 9.99E-01 & 1.36E-01 & 9.99E-01 \\
\hline 25 & 9.99E-01 & 2.04E-01 & 9.98E-01 & 9.99E-01 & 2.04E-01 & 9.98E-01 \\
\hline 26 & 9.92E-01 & $1.76 \mathrm{E}+00$ & 9.83E-01 & $1.00 \mathrm{E}+00$ & 1.76E-01 & $1.00 \mathrm{E}+00$ \\
\hline 27 & 9.99E-01 & 3.37E-01 & 9.97E-01 & $1.00 \mathrm{E}+00$ & $-6.48 \mathrm{E}-02$ & $1.00 \mathrm{E}+00$ \\
\hline 28 & 9.97E-01 & 5.37E-01 & 9.94E-01 & $1.00 \mathrm{E}+00$ & 9.00E-03 & $1.00 \mathrm{E}+00$ \\
\hline 29 & 9.99E-01 & $1.31 \mathrm{E}-01$ & 9.98E-01 & $1.00 \mathrm{E}+00$ & $-3.00 \mathrm{E}-03$ & $1.00 \mathrm{E}+00$ \\
\hline 30 & 9.97E-01 & 7.45E-01 & 9.94E-01 & $1.00 \mathrm{E}+00$ & $-5.99 E-02$ & $1.00 \mathrm{E}+00$ \\
\hline 31 & 9.98E-01 & 4.22E-01 & 9.95E-01 & $1.00 \mathrm{E}+00$ & 6.85E-02 & $1.00 \mathrm{E}+00$ \\
\hline 32 & 9.97E-01 & 5.63E-01 & 9.93E-01 & $1.00 \mathrm{E}+00$ & $-1.51 E-02$ & $1.00 \mathrm{E}+00$ \\
\hline 33 & $1.00 E+00$ & 7.46E-02 & 9.99E-01 & $1.00 E+00$ & 1.12E-02 & $1.00 E+00$ \\
\hline 34 & 9.95E-01 & 1.44E-01 & 9.93E-01 & 9.99E-01 & 3.75E-02 & 9.99E-01 \\
\hline 35 & 9.99E-01 & $2.52 \mathrm{E}-01$ & 9.98E-01 & 9.99E-01 & $1.06 \mathrm{E}-01$ & 9.99E-01 \\
\hline 36 & 9.98E-01 & 2.10E-01 & 9.95E-01 & $1.00 \mathrm{E}+00$ & 3.18E-02 & 9.99E-01 \\
\hline 37 & 9.95E-01 & 1.87E-01 & 9.94E-01 & 9.98E-01 & 9.37E-02 & 9.99E-01 \\
\hline 38 & $9.98 \mathrm{E}-01$ & $2.92 \mathrm{E}-01$ & 9.93E-01 & $1.00 \mathrm{E}+00$ & $1.21 \mathrm{E}-02$ & $9.99 \mathrm{E}-01$ \\
\hline 39 & 9.97E-01 & 5.61E-01 & 9.93E-01 & $1.00 \mathrm{E}+00$ & 6.89E-02 & 9.98E-01 \\
\hline average & 9.97E-01 & 4.34E-01 & 9.94E-01 & $1.00 \mathrm{E}+00$ & 3.27E-02 & 9.99E-01 \\
\hline std deviation & 2.39E-03 & 4.29E-01 & 4.96E-03 & 1.02E-03 & 1.19E-01 & 7.32E-04 \\
\hline $\max$ & $1.00 \mathrm{E}+00$ & $1.76 \mathrm{E}+00$ & $1.00 \mathrm{E}+00$ & $1.00 \mathrm{E}+00$ & 4.58E-01 & $1.00 \mathrm{E}+00$ \\
\hline $\min$ & 9.90E-01 & $-3.75 E-02$ & 9.79E-01 & 9.97E-01 & $-3.45 \mathrm{E}-01$ & 9.96E-01 \\
\hline
\end{tabular}




\section{Appendix C}

Table of Errors (\%) in Maximum Brake Specific 30 second NTE NOx Emissions in MEMS Data Reduced with USB DLM and Corrected USB DLM ECU data

\begin{tabular}{|c|c|c|}
\hline \multirow[b]{2}{*}{ No. } & \multicolumn{2}{|c|}{$\%$ Error } \\
\hline & USB & Corrected \\
\hline 1 & 1.64 & 0.51 \\
\hline 2 & 3.11 & 1.54 \\
\hline 3 & 1.97 & 0.2 \\
\hline 4 & 4.25 & 0.22 \\
\hline 5 & 1.57 & 0.19 \\
\hline 6 & 2.54 & 0.03 \\
\hline 7 & 0.03 & 0.01 \\
\hline 8 & 2.44 & 0.04 \\
\hline 9 & 2.09 & 0.21 \\
\hline 10 & 0.08 & 0.03 \\
\hline 11 & 2.5 & 0.07 \\
\hline 12 & 1.83 & 0.04 \\
\hline 13 & 1.24 & 0.12 \\
\hline 14 & 0.19 & 0.11 \\
\hline 15 & 0.29 & 0 \\
\hline 16 & 0.25 & 0.03 \\
\hline 17 & 0.24 & 0.09 \\
\hline 18 & 0.61 & 0 \\
\hline 19 & 8.93 & 0.28 \\
\hline 20 & 7.75 & 0.03 \\
\hline 21 & 26.6 & 0.05 \\
\hline 22 & 1.69 & 0.08 \\
\hline 23 & 4.02 & 0.11 \\
\hline 24 & 22.08 & 0 \\
\hline 25 & 7.45 & 0.03 \\
\hline 26 & 13.93 & 0.06 \\
\hline 27 & 0.08 & 0.02 \\
\hline 28 & 0.82 & 0 \\
\hline 29 & 0.84 & 0.05 \\
\hline 30 & 47.37 & 0.04 \\
\hline 31 & 8.38 & 0.34 \\
\hline 32 & 1.91 & 0.06 \\
\hline 33 & 27.82 & 0 \\
\hline 34 & 9.48 & 0.01 \\
\hline 35 & 33.86 & 0.24 \\
\hline 36 & 4.34 & 0.06 \\
\hline 37 & 0.83 & 0.11 \\
\hline 38 & 21.53 & 0.03 \\
\hline 39 & 5.33 & 0.46 \\
\hline 40 & 2.39 & 0.1 \\
\hline 41 & 0.24 & 0.02 \\
\hline 42 & 0.07 & 0 \\
\hline 43 & 3.75 & 0.07 \\
\hline 44 & 0 & 0 \\
\hline 45 & 0.44 & 0.09 \\
\hline 46 & 0.31 & 0.02 \\
\hline 47 & 8.66 & 0.09 \\
\hline 48 & 0.83 & 0 \\
\hline 49 & 15.48 & 0.26 \\
\hline 50 & 0.54 & 0.63 \\
\hline 51 & 2.63 & 0.03 \\
\hline 52 & 13.8 & 0.25 \\
\hline 53 & 5.48 & 0.25 \\
\hline 54 & 5.44 & 0.03 \\
\hline 55 & 1.51 & 0.01 \\
\hline Average & 6.24 & 0.13 \\
\hline Maximum & 47.37 & 1.54 \\
\hline
\end{tabular}




\section{Appendix D}

Summary of Time Stamp Errors in J1939 Protocol DLM Data Collected with USB DPA 4 Adapters during 20 Minutes Long FTP Tests.

\section{Adapter 1}

\begin{tabular}{|c|c|}
\hline Test & Time Difference \\
\hline Number & seconds \\
\hline e01155-1 & 14.4 \\
\hline e01155-2 & 20 \\
\hline e01155-3 & 20 \\
\hline e01155-4 & 19.4 \\
\hline e01155-5 & 20.8 \\
\hline e01155-6 & 20 \\
\hline Mean & 19.1 \\
\hline St.Dev. & 2.3 \\
\hline
\end{tabular}

Adapter 2

\begin{tabular}{|c|c|}
\hline Test & Time Difference \\
\hline Number & seconds \\
\hline e1158-1 & 13.8 \\
\hline e1158-2 & 14.2 \\
\hline e1158-3 & 13.8 \\
\hline e1158-4 & 13.8 \\
\hline e1158-5 & 13.4 \\
\hline e1158-6 & 14.2 \\
\hline e1160-4 & 3.2 \\
\hline e1160-5 & 14.2 \\
\hline e1164-1 & 13.8 \\
\hline Mean & 12.7 \\
\hline St.Dev. & 3.6 \\
\hline
\end{tabular}




\section{Appendix E}

\section{Summary of All Correction Factors for ECU Data Collected During In-Use Testing Grouped with Respect to Vehicles and Test Weights}

\begin{tabular}{|c|c|c|c|c|c|}
\hline Test Weight1 & & Test Weight2 & & Test Weight3 & \\
\hline Test \# & Corr. Factor & Test \# & Corr. Factor & Test \# & Corr. Factor \\
\hline \multicolumn{6}{|l|}{ Vehicle 1} \\
\hline 1 & 1.0022 & 5 & 1.0020 & & \\
\hline 2 & 1.0021 & 6 & 1.0022 & & \\
\hline 3 & 1.0020 & 7 & 1.0020 & & \\
\hline \multirow[t]{2}{*}{4} & 1.0022 & 8 & 1.0022 & & \\
\hline & & 9 & 1.0023 & & \\
\hline Mean & 1.0021 & & 1.0021 & & \\
\hline St. Dev & 0.0001 & & 0.0001 & & \\
\hline \multicolumn{6}{|l|}{ Vehicle 2} \\
\hline 10 & 1.0023 & 13 & 1.0023 & & \\
\hline 11 & 1.0022 & 14 & 1.0023 & & \\
\hline \multirow[t]{2}{*}{12} & 1.0024 & 15 & 1.0023 & & \\
\hline & & 16 & 1.0023 & & \\
\hline Mean & 1.0023 & & 1.0023 & & \\
\hline St. Dev & 0.0001 & & 0.0000 & & \\
\hline \multicolumn{6}{|l|}{ Vehicle 3} \\
\hline 40 & 1.0023 & 44 & 1.0023 & & \\
\hline 41 & 1.0022 & 45 & 1.0022 & & \\
\hline 42 & 1.0023 & 46 & 1.0024 & & \\
\hline 43 & 1.0022 & & & & \\
\hline Mean & 1.0023 & & 1.0023 & & \\
\hline St. Dev & 0.0001 & & 0.0001 & & \\
\hline \multicolumn{6}{|l|}{ Vehicle 4} \\
\hline 47 & 1.0072 & 51 & 1.0073 & & \\
\hline 48 & 1.0073 & 52 & 1.0072 & & \\
\hline 49 & 1.0071 & 53 & 1.0073 & & \\
\hline 50 & 1.0072 & 54 & 1.0071 & & \\
\hline & & 55 & 1.0073 & & \\
\hline Mean & 1.0072 & & 1.0072 & & \\
\hline St. Dev & 0.0001 & & 0.0001 & & \\
\hline \multicolumn{6}{|l|}{ Vehicle 5} \\
\hline 17 & 1.0116 & & & & \\
\hline 18 & 1.0121 & & & & \\
\hline 19 & 1.0124 & & & & \\
\hline Mean & 1.0120 & & & & \\
\hline St. Dev & 0.0004 & & & & \\
\hline \multicolumn{6}{|l|}{ Vehicle 6} \\
\hline 20 & 1.0173 & & & & \\
\hline 21 & 1.0177 & & & & \\
\hline 22 & 1.0125 & & & & \\
\hline 23 & 1.0127 & & & & \\
\hline 24 & 1.0129 & & & & \\
\hline Mean & 1.0146 & & & & \\
\hline St. Dev & 0.0026 & & & & \\
\hline \multicolumn{6}{|l|}{ Vehicle 7} \\
\hline 25 & 1.0099 & 29 & 1.0097 & 34 & 1.0094 \\
\hline 26 & 1.0097 & 30 & 1.0096 & 35 & 1.0094 \\
\hline 27 & 1.0099 & 31 & 1.0096 & 36 & 1.0094 \\
\hline \multirow[t]{3}{*}{28} & 1.0097 & 32 & 1.0097 & 37 & 1.0095 \\
\hline & & 33 & 1.0096 & 38 & 1.0094 \\
\hline & & & & 39 & 1.0095 \\
\hline Mean & 1.0098 & & 1.0096 & & 1.0094 \\
\hline St dev & 0.0001 & & 0.0001 & & 0.0001 \\
\hline
\end{tabular}

